University of Rhode Island

DigitalCommons@URI

Open Access Dissertations

1990

\title{
A Unified Performance Analysis of Subspace-Based DOA Estimation Algorithms in Array Signal Processing
}

Fu Li

University of Rhode Island

Follow this and additional works at: https://digitalcommons.uri.edu/oa_diss

\section{Recommended Citation}

$\mathrm{Li}$, Fu, "A Unified Performance Analysis of Subspace-Based DOA Estimation Algorithms in Array Signal Processing" (1990). Open Access Dissertations. Paper 375.

https://digitalcommons.uri.edu/oa_diss/375

This Dissertation is brought to you for free and open access by DigitalCommons@URI. It has been accepted for inclusion in Open Access Dissertations by an authorized administrator of DigitalCommons@URI. For more information, please contact digitalcommons-group@uri.edu. 


\section{A UNIfied Performance ANALYsis of Subspace-Based DOA Estimation AlgorithmS IN ARRAY Signal PROCESSING

\author{
BY \\ FU LI
}

A DISSERTATION SUBMITTED IN PARTIAL FULFILLMENT OF THE REQUIREMENTS FOR THE DEGREE OF DOCTOR OF PHILOSOPHY

IN

ELECTRICAL ENGINEERING

UNIVERSITY OF RHODE ISLAND

1990 
DOCTOR OF PHILOSOPHY DISSERTATION

OF

FU LI

Approved:

Dissertation Committee

Major Professor

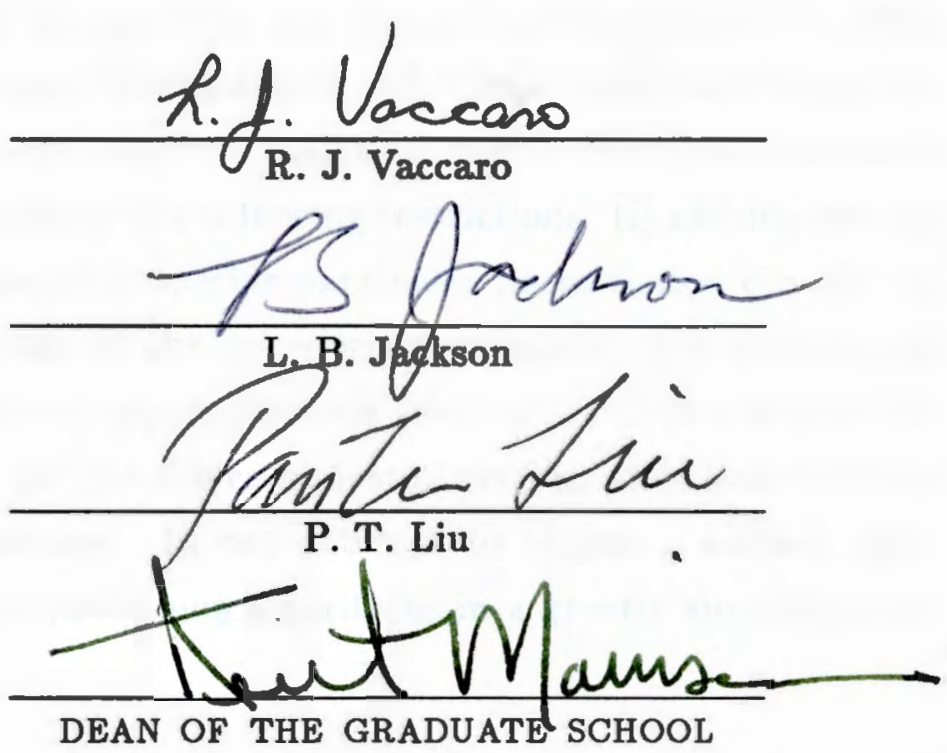

UNIVERSITY OF RHODE ISLAND

1990 


\section{Abstract}

In the last decade, the subspace approach has found prominence in the problem of estimating directions of arrival using an array of sensors. Many subspace methods have been proposed and improved; the most attractive ones among these are MUSIC, Min-Norm, State-Space Realization (TAM) and ESPRIT. However, performance analyses are required for justifying and comparing these methods before applying them. Early performance justifications and comparisons were based on simulations. In recent years, many excellent analytical studies have been reported, but these studies have one or more of the following restrictions: (i) assume asymptotic measurements, (ii) analyze some specific parameter perturbation directly instead of through the perturbation of the appropriate subspace, (iii) evaluate individual algorithms using different approximations (so it is hard to compare the analyses of different methods), (iv) involve complicated mathematics and statistics which result in difficult expressions. In our attempt to obtain a unified, nonasymptotic analysis to subspace processing algorithms in a greatly simplified and self-contained fashion, we

1. classify these algorithms into category by the subspace they use - orthogonalsubspace processing and signal-subspace processing. We then derive expressions for the first-order perturbation of the signal and orthogonal subspaces using a matrix approximation technique. These formulas provides a common foundation for our analysis of all the DOA estimation algorithms mentioned above. 
2. define three approaches by the numerical procedure these algorithms exploit - extrema-searching, polynomial-rooting approach, matrix-shifting approach. We establish a common model for each approach and analyze these common models (instead of individual algorithms), and specialize the results for each algorithm.

3. provide a first-order relationship between subspace perturbations and direction-of-arrival perturbations.

4. use the perturbation formulas to derive variance expressions for DOA estimates for all the algorithms. We make the comparisons and discussions among these algorithms and approaches with our theoretical prediction and numerical simulations.

The tractable formulas derived in this analysis provide insight into the performance of the algorithms. Simulations verify the analysis. 


\section{Acknowledgments}

I would like to express my sincere gratitude and deepest appreciation to my major advisor, Dr. Richard J. Vaccaro, for his constant support, encouragement, enthusiasm and patience in guiding this research. His probing comments and insightful suggestions are invaluable resources which made this research all possible. He certainly deserves much credit.

I am deeply grateful to Drs. Leland B. Jackson and P-T. Liu for their guidance and support as the thesis committee members, and Drs. G. Faye BoudreauxBartels, James T. Lewis, E. R. Suryanarayan and H. Ozbay for their services in various examinations in my Ph.D. program.

I also wish to thank Drs. Donald W. Tufts, Ramdas Kumaresan, Steven M. Kay and other members of faculty for their contribution in my graduate education. I never hesitated to knock their doors whenever I had questions. Thanks are also due to Zhigang Fan, Alex C. Kot, Ivars Kirsteins, Eleftherios Maragakis and other fellow graduate students with whom I had great pleasure to interact with. I feel specially privileged to have had my Ph.D. program in University of Rhode Island which has exceptional expertise in signal processing.

I would like to gratefully acknowledge all the people who helped me during my Ph.D. program.

This dissertation is dedicated to my wife, daughter and parents. I will forever be indebted to them for their love and sacrifices throughout.

This research was supported in part by the Office of Naval Research under grant N00014-90-J1283 and by the Army Research Office under contract DAAL03-86-K0108 


\section{Preface}

This dissertation is based on research in the area of array signal processing conducted under the supervision of Dr. Richard J. Vaccaro. Many of the results presented in this dissertation appear in conference proceedings and in manuscripts accepted by or submitted to refereed journals, and all of them are co-authored with Dr. Richard J. Vaccaro and some also co-authored with Dr. Donald W. Tufts.

Chapter 4 is based on results in

- "Min-Norm Linear Prediction for Arbitrary Sensor Arrays" Proc. IEEE International Conf. on Acoustics, Speech and Signal Processing'89, Vol.4, pp.2613-2616, Glasgow, Scotland, May, 1989

- "A Matrix Approximation Approach to MUSIC Perturbation Analysis", Proc. 23rd Annual Conf. Information Sciences and Systems, pp.477-481, Johns Hopkins, March 1989

- "Non-Asymptotic Perturbation Analysis of Orthogonal-Subspace Processing with Arbitrary Array Geometry", to appear in IEEE Trans. on Aerospace and Electronic Systems.

Some of the results in Chapter 5 appear in

- "Perturbation Analysis of Subspace-Based Polynomial-Rooting Algorithms for DOA Estimation”, Proc. 24nd Annual Conf. Information Sciences and Systems, Princeton, March, 1990

The results of in Chapter 6 are in 
- "Unified Performance Analysis of Subspace-Based Estimation Algorithms" Proc. International Conf. on Acoustics, Speech and Signal Processing'90, Vol.5, pp.2575-2578.

- "Performance Analysis of The State Space Realization (TAM) and ESPRIT Algorithms for DOA Estimation", submitted to IEEE Trans. on Antenna and Propagation.

A brief summary of this complete research can be found in

- "Analytical Performance Prediction of Subspace-Base Algorithms for DOA Estimation", Proc. 2nd International Workshop on SVD and Signal Processing, pp.161-168, Kingston, RI, June, 1990.

This research was extended to analyze the performance in the presence of sensor error as appears in

- "Statistical Comparison of Subspace-Based DOA Estimation Algorithms In the Presence of Sensor Errors", Proc. 5th IEEE ASSP Workshop on Spectrum Estimation and Modeling, Rochester, NY, October, 1990.

In course of this research, we also achieved some results in two dimensional array signal processing

- "Frequency-Wavenumber Estimation by State-Space Realization", Proc. 23rd Annual Conf. Information Sciences and Systems, p.609, Johns Hopkins, March 1989. 


\section{Contents}

Abstract $\quad$ ii

Acknowledgments $\quad$ iv

Preface $\quad$ v

Table of Contents vii

List of Tables $\quad$ xi

List of Figures $\quad$ xii

1 Introduction 1

1.1 Statement of the Problem ................... 1

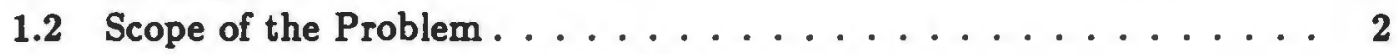

1.3 Summary of the Other Works ................ 3

1.4 Significance and Contributions ............... 5

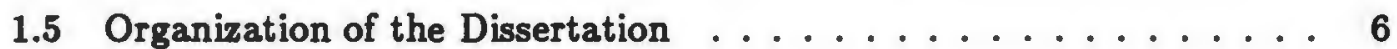

1.6 Nomenclature ...................... 8

2 Historical Review and Algorithm Classification 10

2.1 Assumption and Signal Model . . . . . . . . . . . . . . 10

2.2 Pre-Subspace Methods . . . . . . . . . . . . . . . 12

2.2.1 Classical Fourier Analysis - Periodogram ......... 12

2.2.2 Conventional Beamforming ............. 13 
2.2.3 Maximum Entropy Method . . . . . . . . . . . . 14

2.2.4 Minimum Variance Method . . . . . . . . . . . . 15

2.3 Subspace Based Algorithms . . . . . . . . . . . . . 16

2.3.1 Subspace Decomposition . . . . . . . . . . . 16

2.3 .2 MUSIC $\ldots \ldots \ldots \ldots \ldots \ldots \ldots \ldots \ldots \ldots \ldots$

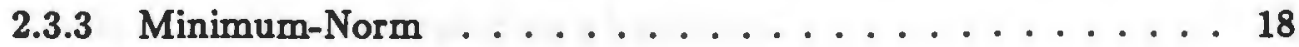

2.3.4 Root-MUSIC Algorithm . . . . . . . . . . . . . 19

2.3.5 State-Space Realization . . . . . . . . . . . . 19

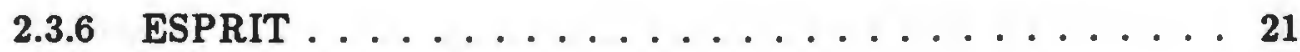

2.3.7 Matrix-Pencil Method . . . . . . . . . . . . 23

2.3 .8 Remark . . . . . . . . . . . . . . . . 24

2.4 Classification of The Subspace Algorithms . . . . . . . . 24

2.4.1 Classification by Subspace . . . . . . . . . . . . . 24

2.4.2 Classification by Numerical Procedure . . . . . . . . 25

3 Perturbation of Subspaces $\quad 26$

3.1 Introduction . . . . . . . . . . . . . 26

3.2 Subspace Review . . . . . . . . . . . . . . . 27

3.3 Perturbation of Orthogonal-Subspace . . . . . . . . . . 30

3.4 Perturbation of Signal-Subspace . . . . . . . . . . . . 32

3.5 Summary . . . . . . . . . . . . . . . . 33

4 Performance of Extrema Searching Algorithms 34

4.1 Introduction . . . . . . . . . . . . . . . 34

4.2 Common Model . . . . . . . . . . . . . . . . 35

4.3 Perturbation of the Angle Estimates . . . . . . . . . 36

4.4 Statistical Performance. . . . . . . . . . . . . . . . 40

4.5 Analytical Comparison of MUSIC and Min-Norm . . . . . . . . 41

4.6 Numerical Examples . . . . . . . . . . . . . . . . . 42

4.7 Summary $\ldots \ldots \ldots \ldots \ldots \ldots \ldots \ldots \ldots \ldots \ldots \ldots$ 
5 Performance of Polynomial Rooting Algorithms 46

5.1 Introduction . . . . . . . . . . . . . 46

5.2 Common Model . . . . . . . . . . . . . . . . . . 47

5.3 Perturbation of the Angle Estimates . . . . . . . . . 49

5.4 Statistical Performance . . . . . . . . . . . . 55

5.5 Relation to Extrema-Searching Algorithms . . . . . . . . . 56

5.6 Mean-Squared Error of Root Perturbation . . . . . . . . . 56

5.7 Numerical Examples . . . . . . . . . . . . . . . . 59

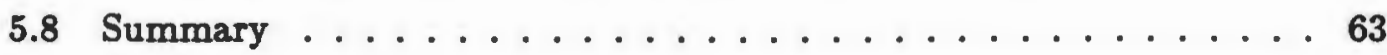

6 Performance of Matrix Shifting Algorithms 64

6.1 Introduction . . . . . . . . . . . . . . 64

6.2 Common Model . . . . . . . . . . . . . . . . . . 65

6.3 Perturbation of the Angle Estimates . . . . . . . . . 66

6.4 Statistical Performance. . . . . . . . . . . . . . 71

6.5 Numerical Examples . . . . . . . . . . . . . 73

6.6 Summary . . . . . . . . . . . . . . . . 74

7 Comparison and Conclusion $\quad 77$

7.1 Introduction . . . . . . . . . . . . 77

7.2 Summary of Analysis . . . . . . . . . . . . . 77

7.3 Comparative Examples . . . . . . . . . . . . . . . . 79

7.4 Concluding Remarks . . . . . . . . . . . . . 84

$\begin{array}{ll}\text { A Mean-Squared Error Calculation } & 85\end{array}$

$\begin{array}{ll}\text { B Pisarenko's Method } & \mathbf{8 7}\end{array}$

C Perturbation of Angle and Root 90

D Cramer-Rao Bound Calculation $\quad 92$

$\begin{array}{ll}\text { References } & 93\end{array}$ 
Bibliography 


\section{List of Tables}

4.1 Parameters of mean-squared error for Extrema Search Algorithms . 42

5.1 Parameters of mean-squared error for Polynomial Rooting Algorithms 60

6.1 Parameters of mean-squared error for Matrix Shifting Algorithms . . 73

7.1 Parameters of mean-squared error for all algorithms . . . . . . 80 


\section{List of Figures}

4.1 Uniform circular array . . . . . . . . . . . . . . 44

4.2 Null-spectrum function of Extrema Search Approach . . . . . . 44

4.3 RMSE vs. SNR for MUSIC and Min-Norm at source 1 (.2 rads) . . 45

4.4 RMSE vs. SNR for MUSIC and Min-Norm at source 2 (.5 rads) . . 45

5.1 Uniform line array . . . . . . . . . . . . . 60

5.2 RMSE vs. SNR for Min-Norm and Root-MUSIC at source 1 (.2 rads) 61

5.3 RMSE vs. SNR for Min-Norm and Root-MUSIC at source $2(.5 \mathrm{rads}) 61$

5.4 Scattergram of Estimated Roots for Min-Norm . . . . . . . . . 62

5.5 Scattergram of Estimated Roots for Root-MUSIC . . . . . . . . . 62

6.1 ESPRIT Array geometry from $[35] \ldots \ldots \ldots \ldots \ldots$

6.2 Scattergram of Estimated eigenvalues . . . . . . . . 75

6.3 RMSE vs. SNR for SSR and ESPRIT at source 1 (.2 rads) $\ldots .76$

6.4 RMSE vs. SNR for SSR and ESPRIT at source $2(.5 \mathrm{rads}) \ldots \ldots 76$

7.1 RMSE vs. Signal-to-Noise Ratio . . . . . . . . . . . . 82

7.2 RMSE vs. Source Separation . . . . . . . . . . . 82

7.3 RMSE vs. Number of Sensors . . . . . . . . . . . . 83

7.4 RMSE vs. Number of Snapshots $\ldots \ldots \ldots \ldots \ldots \ldots$ 


\section{Chapter 1}

\section{Introduction}

\subsection{Statement of the Problem}

This dissertation analyzes the statistical performance of subspace processing algorithms for estimating directions-of-arrival (DOA) from sensor array signals.

Given an array of $L$ sensors, the problem is to estimate the directions of $P$ uncorrelated plane-wave signals which simultaneously impinge on the array. By taking measurements simultaneously at all sensors, at $M$ different instants in time, an $L \times M$ (sensor versus snapshot) data matrix can be formed. The first step in the subspace approach to the estimation of directions-of-arrival is to perform an eigenvalue decomposition on the covariance estimates of the data matrix or, equivalently, perform a singular value decomposition directly on the data matrix. The second step is to obtain a signal-subspace spanned by the eigen (singular) vectors associated with $P$ largest eigen (singular) values and an orthogonal-subspace spanned by the eigen (singular) vectors associated with $L-P$ smallest eigen (singular) values. Since these two subspaces are orthogonal complements of each other, either of them can be used to estimate the directions of arrival. After acquiring the appropriate subspace, each method estimates the interesting parameters in a different fashion of exploiting the underlying signal propagation model.

In the presence of the noise introduced by observation, quantization, etc., the direction-of-arrival estimators cannot be error-free. Different subspace methods 
have different sensitivities to perturbations. An analytical justification and comparison of their performances is therefore important. Based on a comprehensive study of their statistical error, we develop a unified approach to the performance analyses of these subspace methods.

\subsection{Scope of the Problem}

Array signal processing has been a very active research field for several decades motivated by the increasing demand for radar, sonar, radio, seismic, speech and other signal processing in applications of electronic surveillance, telecommunications, astronomy, geographics, oceanographics, and bio-medical science. In the last ten years, the subspace approach has found its prominence in the problem of estimating directions of arrival, which is one of the most important topics in array processing. Many subspace methods have been proposed and improved. Numerous papers and reports were documented in the literature every year. However performance analyses are required for justifying and comparing these methods for the purpose of applying them. Our study of statistical performance includes a high SNR perturbation analysis of the subspaces and of many different subspace-based DOA estimation algorithms. A brief list of the topics covered in this dissertation is given below:

- Historic review and classification of algorithms based on subspaces and on numerical procedures [Chapter 2]

- Perturbation analyses of the signal subspace and orthogonal (noise) subspace [Chapter 3]

- Perturbation analyses of extrema-searching algorithms such as MUSIC and Extended Min-Norm [Chapter 4]

- Perturbation analyses of polynomial-rooting algorithms such as Root-MUSIC and Min-Norm [Chapter 5] 
- Perturbation analyses of matrix-shifting algorithms such as State-Space Realization and ESPRIT [Chapter 6]

- Unified expression of mean-squared error and a numerical example of comparison [Chapter 7]

The first topic addresses the perturbation of the subspaces, which provides the common foundation for the analyses of various algorithms. We then classify those various algorithms into three categories based on their estimation procedures: extrema-searching, polynomial-rooting and matrix-shifting. The analyses of these types of algorithms form the topics of latter three chapters.

\subsection{Summary of the Other Works}

Motivated by the increasing popularity of subspace processing algorithms in DOA estimation, many researchers turned to study the performance of these algorithms. Early performance justification and comparisons are based on simulations [1], which cannot avoid dependence on the selection of simulation examples. In recent years, analytical performance justification and comparisons have been actively developed. Among many other excellent analyses, the following works have specific contributions:

- Kaveh and Barabell [2] used the first and second moments of the perturbed signal eigenvalues and eigenvectors to derive a first order approximation to the mean and variance of the estimated spectrum for MUSIC, and the mean for Min-Norm based on first order perturbation of the null-spectrum in the signal neighborhoods.

- Porat and Friedlander $[3,4]$ later developed a similar approach for MinNorm and MUSIC using first-order Taylor series approximations of estimated parameters around their true values. 
- Clergeot, Tressens and Ouamri [5] analyzed the performances of MUSIC and Min-Norm based on a first-order linear perturbation method to calculate the perturbed signal eigenvectors.

- Hua and Sarkar [6] presented an analysis of Min-Norm by calculating the perturbation of the pseudo-inverse of the truncated data matrix.

- Bhaskar Rao presented separate statistical analyses of Min-Norm [7, 8], RootMUSIC [9], TAM [10] and ESPRIT [9].

- Stoica and Nehorai $[11,12,13]$ derived an expression for the covariance of MUSIC estimate of the direction of arrival and used it for performance comparison of MUSIC with the Maximum-Likelihood Method and the CramerRao Bound.

- Farrier, Jeffries and Mardani $[14,15]$ presented a second-order analysis to the expected value of MUSIC spectrum.

- Tufts, Vaccaro and Kot [16] proposed a backward error analysis to Min-Norm Linear Prediction of time series using matrix approximation.

Many of these excellent methodologies and results, especially the simplicity and efficiency of the backward error analysis, inspired this research. However, despite of their great successes, most of these performance analyses have one or more of the following restrictions:

- assume asymptotic measurements which may not be realistic in practice,

- analyze some specific parameter perturbation directly instead of the perturbation of the appropriate subspace,

- evaluate individual algorithms using different approximations, and so it is hard to compare the analyses of the different algorithms.

- involve complicated mathematics and statistics which result in complicated expressions. 
Therefore, the goal of this research was to provide a non-asymptotic, unified and simplified performance analysis.

\subsection{Significance and Contributions}

The novelty of the performance analysis presented in this dissertation is that it encompasses a variety of problems and provides a unified framework. A theoretically and computationally simple analysis is common to all the subspace methods, which are formulated in a general framework. In the next few paragraphs, the main results of the present work are listed:

1. A unified approach is developed for the performances of all the subspace methods including Pisarenko's method, MUSIC, Min-Norm Linear Prediction, Root-MUSIC, State-Space Realization, ESPRIT and Matrix-Pencil. The expressions for the perturbation of the signal-subspace and of orthogonalsubspace are derived, which provide a common foundation for our analysis of all the DOA estimation algorithms mentioned above. This approach is also applicable to several other methods beyond subspace processing methods.

2. The performance is analyzed in a greatly simplified and self-contained fashion, which provides the tractable formulae and insight for the algorithms. Unnecessarily complicated mathematics and statistics have been successfully avoided.

3. The performance is analyzed for the case in which only a finite number of measurements are available, which is the common restriction for many array signal processing applications owing to some practical limitation, for instance, nonstationarity. This work makes the assumption of high signal-to-noise ratio which is reasonable in some practical situations, and is also of theoretical interest. Furthermore, the analyses are shown to agree with simulations down to a fairly low signal-to-noise ratio near threshold, which indicates the high SNR assumption is only sufficient but may not be necessary. 
4. To achieve the goal, we have also categorized three different types of subspace methods by their procedure of solving the problem: extrema-searching, polynomial-rooting and matrix shifting.

5. We have derived a unified framework for different algorithms in each category, which is a necessary step toward unifying all the subspace methods.

6. As a verification, the predicted performance from our analysis is shown to be in good agreement with measured performance from simulations over a wide range of signal-to-noise ratios, over various array geometries, over different observation lengths, over various angular separations.

During the course of this work, the application of the Min-Norm Linear Prediction technique to a sensor array of arbitrary geometry has been documented. Previous papers describing the Min-Norm technique apply it to linear arrays, and use a polynomial rooting procedure to obtain the estimates. It was shown in [17] that the Min-Norm technique is applicable to arrays of arbitrary geometry by using an extrema-searching procedure similar to the MUSIC algorithm.

\subsection{Organization of the Dissertation}

This dissertation is arranged as outlined below:

Chapter 2. Provides a comprehensive review of array processing tracing back to its origin in time series analysis in the 18th century when Prony initiated the linear prediction of time series, up to and including the state-of-art with emphasis on an historical perspective of major trends. The classifications based on subspaces and numerical procedures of the algorithms are described.

Chapter 3: The concept of subspace is introduced using the principal component decomposition. An equivalence of the covariance based approach (which uses the eigendecomposition) and the direct data based approach (which uses the singular value decomposition) is discussed. The perturbation analyses of subspaces 
developed in this chapter will play a central role through the whole dissertation. The perturbation of the orthogonal-subspace is presented through a first-order perturbation expansion. However, it is interesting to see that the perturbation of the signal-subspace is not derived in the same way, but rather from the orthogonality between the orthogonal subspace and signal subspace. The results in this chapter provide a common tool with which to analyze all the subspace methods.

Chapter 1: The extrema-searching approach of the orthogonal-subspace based methods is defined, reviewed and analyzed. The application of Min-Norm Linear Prediction to an array of arbitrary geometry (called Search Min-Norm) is presented in this chapter. A unified model for MUSIC and Search Min-Norm Linear Prediction is derived, followed by the perturbation analysis of this common model. The perturbation of MUSIC and Search Min-Norm Linear Prediction are deduced as special cases of this common model and then compared with each other.

Chapter 5: The polynomial-rooting approach of the orthogonal-subspace based methods is defined, reviewed and analyzed. A common spectral polynomial model for Root-MUSIC and Min-Norm Linear Prediction is established as in the previous chapter for a unified analysis. The Root-MUSIC and Min-Norm Linear Prediction are therefore analyzed as special cases of the unified analysis. The relation between root perturbation and the angle perturbation is also utilized. The comparison is made for Root-MUSIC and Min-Norm Linear Prediction.

Chapter 6: The matrix-shifting approach for the signal-subspace based methods is defined, reviewed and analyzed. A common model for State-Space Realization and ESPRIT is found for a unified analysis. The analysis of the matrix-shifting approach is different from the other approaches analyzed in the previous two chapters. We achieve the tractable formulas through analyzing system matrices and their principal eigenvalues. The relationship between perturbations of eigenvalues and of directions of arrival is derived.

Chapter 7: A unified expression of mean-squared error for DOA estimation with parameters specializing it for different algorithms is presented, followed by a 
numerical example comparing all the algorithms with respect to variations of signalto-noise ratio, source separation, number of sensors and number of snapshots. The concluding remarks of this research is made at the end of the chapter.

\subsection{Nomenclature}

- Principal symbols

S- signal matrix $\mathbf{S}=[\mathbf{s}(1), \ldots, \mathbf{s}(M)]$

$\mathrm{N}$ - noise matrix $\mathbf{N}=[\mathbf{n}(1), \ldots, \mathrm{n}(M)]$

$\mathbf{Y}$ - data observed at sensor array $\mathbf{Y}=[\mathbf{y}(1), \ldots, \mathbf{y}(M)]$

R- covariance matrix of data

P - spatial null-spectrum of the data

$\boldsymbol{\Sigma}, \mathbf{U}, \mathbf{V}$ - singular values and associated left- and right- singular vectors of data matrix $\left(\Sigma=\operatorname{diag}\left\{\sigma_{i}\right\}\right)$

$\boldsymbol{\Lambda}, \mathbf{E}$ - eigenvalues and associated eigenvectors covariance matrix $(\boldsymbol{\Lambda}=$ $\left.\operatorname{diag}\left\{\lambda_{i}\right\}\right)$

$L, M, P$ - number of sensors, snapshots, sources

$i, j, k$ - index of sensors, snapshots, sources

$\sigma_{n}^{2}$ - power of observation noise

$\lambda_{c}-$ center wavelength of narrow-band plane-wave signal

$d$ - the distance between adjacent sensors

$\theta$ - direction-of-arrival plane-wave signal

$\omega$ - spatial frequency $\omega=\frac{2 \pi d}{\lambda_{a}} \sin \theta$

$a(\theta)$ - signal manifold (or steering) vector

- Matrix notation

$[\cdot]$ - signal-subspace affiliated with $[\cdot]$ 
$[\cdot]_{0}$ - orthogonal-subspace affiliated with $[\cdot]$

$[\cdot]$ - noise-corrupted quantity of $[\cdot]$

$\Delta[\cdot]$ - perturbed quantity of $[\cdot]$

$\Re[\cdot]$ - real part of $[\cdot]$

$\Im[\cdot]$ - imaginary part of $[\cdot]$

$E[\cdot]$ - expectation of $[\cdot]$

Superscripts $\mathrm{H}, \mathrm{T}$ and ${ }^{*}$ - conjugate transpose, transpose and conjugation of the matrix

Superscripts $\dagger$ and $\sharp$ - left- and right- pseudo-inverse of the matrix

- Chapter 4

W- weighting matrix

d- linear prediction error vector

$x, y$-sensor position coordinates for an array of arbitrary geometry.

- Chapter 5

$z-\mathrm{Z}$ transform domain component $z=e^{j \frac{2 \pi d}{\lambda_{\theta}} \sin \theta}$

$r$ - root of spectral polynomial

$C_{k}$-coefficient between angle and and imaginary part of root (or eigenvalue)

$$
C_{k}=\frac{\lambda_{c}}{2 \pi d \cos \theta_{k}}
$$

- Chapter 6

F- transition matrix in SSR and ESPRIT

O, C- observability and controllability matrices in SSR

$\mathbf{X}$ - data observed at $\mathbf{x}$ subarray $X=[\mathbf{x}(1), \ldots, \mathbf{x}(P)]$

Z- data observed at $\mathrm{z}$ subarray $Z=[\mathbf{z}(1), \ldots, \mathbf{z}(M)]$

$\lambda, \mathbf{u}, \mathbf{v}-$ eigenvalue and associated left- and right- eigenvector of $\Delta \mathbf{F}$ 


\section{Chapter 2}

\section{Historical Review and Algorithm Classification}

\subsection{Assumption and Signal Model}

Given an array of $L$ sensors and $M$ snapshots of measurements taken at each sensor, the objective is to estimate the directions of $P$ uncorrelated plane-wave signals which simultaneously impinge on the array.

The following assumptions are made throughout the whole dissertation unless being otherwise stated.

- The $L$ array sensors are identical to each other. The number of sensors is greater than the number of signal sources $(L>P)$.

- The $P$ signal wavefronts $s_{k}$ are narrow-band (with the center wavelength $\lambda_{c}$ ) plane-waves (far-field), uncorrelated from source to source.

- The observation noise $n_{i}$ at each sensor is additive complex white-Gaussian with zero-mean and variance $\sigma_{n}^{2}\left(\frac{1}{2} \sigma_{n}^{2}\right.$ for independent real and imaginary parts), independent from sensor to sensor and from the signals.

Under the above generic assumptions, the signal arriving at the $i$-th sensor located at $\left(x_{i}, y_{i}\right)$ (for $\left.i=1, \ldots, L\right)$ at time $t$ is 


$$
\begin{aligned}
& y_{i}(t)= \sum_{k=1}^{P} e^{j \frac{2 \pi}{\lambda_{i}}\left(x_{i} \sin \theta_{k}+y_{i} \cos \theta_{k}\right)} s_{k}(t) \\
&=\left(\begin{array}{lll}
e^{j \frac{2 \pi}{\lambda}\left(x_{i} \operatorname{\theta in} \theta_{1}+y_{i} \cos \theta_{1}\right)} & \ldots & e^{j \frac{2 \pi}{\lambda}\left(x_{i} \sin \theta_{P}+y_{i} \cos \theta_{P}\right)}
\end{array}\right)\left(\begin{array}{c}
s_{1}(t) \\
\vdots \\
s_{P}(t)
\end{array}\right), \\
& i=1, \ldots, L ; \quad k=1, \ldots, P,
\end{aligned}
$$

where $s_{k}(t)$ is the $k$-th narrowband signal (with center wavelength $\lambda_{c}$ ) arriving at an angle $\theta_{k}$. The signals arriving at all the sensors at time $t$ are

$$
y(t)=\left(\begin{array}{ccc}
e^{j \frac{2 \pi}{\lambda}\left(\varepsilon_{1} \sin \theta_{1}+y_{1} \cos \theta_{1}\right)} & \ldots & e^{j \frac{2 \pi}{\lambda}\left(x_{1} \sin \theta_{P}+y_{1} \cos \theta_{P}\right)} \\
\vdots & \ddots & \vdots \\
e^{j \frac{2 \pi}{\lambda}\left(x_{L} \sin \theta_{1}+y_{L} \cos \theta_{1}\right)} & \ldots & e^{j \frac{2 \pi}{\lambda}\left(x_{L} \sin \theta_{P}+y_{L} \cos \theta_{P}\right)}
\end{array}\right)\left(\begin{array}{c}
s_{1}(t) \\
\vdots \\
s_{P}(t)
\end{array}\right)
$$

or in vector form

$$
\mathbf{y}(t) \stackrel{\text { def }}{=}\left(\mathbf{a}\left(\theta_{1}\right) \ldots a\left(\theta_{P}\right)\right) \mathbf{s}(t) \stackrel{\text { def }}{=} \mathbf{A}(\theta) \mathbf{s}(t)
$$

where $\mathbf{A}(\theta)$ represents the array characteristics. $M$ snapshots of the signal can be used to form a data matrix as

$$
\mathbf{Y}=\left(\begin{array}{ccc}
y_{1}(1) & \ldots & y_{1}(M) \\
\vdots & \ddots & \vdots \\
y_{L}(1) & \cdots & y_{L}(M)
\end{array}\right)=\mathbf{A}(\theta) \mathbf{S}
$$

where

$$
\mathbf{S} \stackrel{\text { def }}{=}\left(\begin{array}{lll}
\mathbf{s}(1) & \ldots & \mathbf{s}(M)
\end{array}\right)=\left(\begin{array}{ccc}
s_{1}(1) & \ldots & s_{1}(M) \\
\vdots & \ldots & \vdots \\
s_{P}(1) & \ldots & s_{P}(M)
\end{array}\right)
$$

In the case of uniform line array, the signal manifold matrix $\mathbf{A}(\theta)$ has a Vandemonde structure

$$
\mathbf{A}(\theta)=\left(\begin{array}{ccc}
1 & \ldots & 1 \\
e^{j \frac{2 \pi d}{\lambda} \sin \theta_{1}} & \ldots & e^{j \frac{2 \pi d}{\lambda} \sin \theta_{P}} \\
\vdots & \ddots & \vdots \\
e^{j \frac{2 \pi d}{\lambda}(L-1) \sin \theta_{1}} & \ldots & e^{j \frac{3 \pi d}{\lambda}(L-1) \sin \theta_{P}}
\end{array}\right) \stackrel{\text { def }}{=}\left(\begin{array}{cccc}
1 & 1 & \ldots & 1 \\
z_{1} & z_{2} & \ldots & z_{P} \\
\vdots & \vdots & \ddots & \vdots \\
z_{1}^{L-1} & z_{2}^{L-1} & \ldots & z_{P}^{L-1}
\end{array}\right)
$$


where

$$
z_{k}=e^{j \frac{2 \pi d}{\lambda_{e} \sin l_{k}}} .
$$

In the presence of additive observation noise, the data matrix is

$$
\tilde{\mathbf{Y}}=\mathbf{Y}+\mathbf{N}
$$

The covariance matrix of the array data vector $y$ is

$$
\mathbf{R}=E\left[\mathbf{y} \mathbf{y}^{H}\right]=\mathbf{A} \mathbf{R}, \mathbf{A}^{H}
$$

where $\mathbf{R}_{\mathbf{s}}=E\left[\mathbf{s s}^{B}\right] \quad(P \times P)$ is the covariance matrix of the signal vector. In practice, the covariance matrix is estimated from

$$
\tilde{\mathbf{R}}=\frac{1}{M} \tilde{\mathbf{Y}} \tilde{\mathbf{Y}}^{H} .
$$

\subsection{Pre-Subspace Methods}

The theory of array processing has its origin in time-series analysis and spectral estimation, so it can be traced back to the 18th century when Gaspard Riche, the Baron de Prony published his work on fitting a sum of sinusoids to data in 1795 [18]. But most of the Pre-Subspace Methods are restricted to the case of a uniform line array.

\subsubsection{Classical Fourier Analysis - Periodogram}

In 1898, Schuster introduced the idea of the periodogram for determining the periodicities of meterological phenomenon. At that time the calculation of the periodogram was computationally a very expensive procedure. But with the advent of digital computers and after the discovery of fast Fourier transform (FFT) algorithm [19], the periodogram has become the standard method for frequency estimation and is probably the most frequently used method even in recent days. When the noise in the observed data is Gaussian distributed, the periodogram has been shown to produce a maximum likelihood estimate of the frequency when only a 
single sinusoid is present. But the periodogram fails to distinguish between two closely spaced frequencies (separated by less than $\frac{2 \pi}{N T}$, where $N$ is the number of observations and $T$ is the observation interval) and provides only a single frequency estimate instead of two. The inability to separate the two closely spaced sinusoids is due to the length of the data record $\left(N<\frac{2 \pi}{T}\left|\sin \theta_{k}-\sin \theta_{j}\right|\right)$, and is not related to the signal-to-noise ratio. When enough data is available, the periodogram of sufficiently zero padded data sequence can provide reasonably good estimates.

\subsubsection{Conventional Beamforming}

The conventional method of mapping the monochromatic field power as a function of angle from the array axis is the phase-to-phase beamforming operation, which consists of phasing the narrow band sensor outputs to a set of plane wavefronts whose directions span those of interest. It employs a procedure known as delayand-sum processing in an attempt to steer a beam in a particular direction. The concept is based on the physics of wave propagation phenomenon whereby sensors at different locations with respect to the incoming plane wave receive (nearly) the same signal, but with different time-delays with respect to some reference due to the propagation path lengths. If delays are inserted at each of the sensors to exactly compensate for the propagation and differential receiver delays, and outputs of the delay elements summed to form a scalar output, the energy in the signal waveforms will add voltage-wise while the measurement noise in each sensor, being uncorrelated from sensor to sensor, adds power-wise. The steering vector can be solved from a nonlinear constrained maximization problem of finding a unit-norm vector $w$ such that the array output power is maximum. Such a steering vector has a form of

$$
w(\theta)=\frac{1}{L}\left[e^{-j \omega_{0} r_{1}(\theta)}, \ldots, e^{-j \omega_{0} r_{L}(\theta)}\right] .
$$

The idea behind conventional beamforming as a DOA estimation technique can be seen in the weight vector construction. Given measurements from the sensor array, and the set of known (calculated or calibrated) weight vectors, scan the region of $\theta$-space in which the source may present (i.e., $\forall \theta \in \Theta$ ) for relative maximal output 
power

$$
P_{B F}(\theta)=w(\theta)^{H} \mathbf{R w}(\theta) .
$$

The values of $\theta$ which maximizes $P_{B F}(\theta)$ are DOA estimates.

The beamforming technique is conceptually and computationally simple, but in the presence of multiple sources, the method breaks down completely because maxima are no longer guaranteed to be at the true source DOAs.

\subsubsection{Maximum Entropy Method}

In 1967, Burg [20] pointed out that the conventional beamforming has the problem of utilizing a truncated correlation estimate in lag space which results in a smoothing of true spectral function in frequency space. The extrapolated correlation lags should commit least with respect to observed data. Motivated by the results of information theory, he proposed a constrained maximization problem, i.e., find a $\hat{P}(f)$ which maximizes

$$
\max _{P(f)} \int_{-W}^{W} \log P(f) d f
$$

subject to the constraints

$$
r(k \Delta \tau)=\int_{-W}^{W} P(f) e^{j 2 \pi f k \Delta r} d f, \quad \text { for } \quad k=-L, \ldots, L,
$$

where the $2 L+1$ correlation lags are assumed known and $\Delta \tau=1 / 2 W$. The resulting variational problem obtained by using standard Lagrange multiplier techniques can be written as follows:

$$
\delta\left[\int_{-W}^{W} \log P(f) d f-\sum_{k=-L}^{L} \lambda_{k}\left\{\int_{-W}^{W} P(f) e^{j 2 \pi f k \Delta r} d f-r(k \Delta \tau)\right\}\right]=0
$$

The solution is straightforward

$$
\hat{P}(f)=\frac{\mathbf{u}_{1}^{T} \mathbf{R}^{-1} \mathbf{u}_{1}}{\left\|\mathbf{u}_{1}^{T} \mathbf{R}^{-1} \mathbf{a}\right\|^{2}}
$$

where $\mathbf{R}$ is the Toeplitz correlation matrix of known lags, and

$$
u_{1} \stackrel{\text { def }}{=}[1,0, \ldots, 0]^{T},
$$




$$
\text { a def }=\left[1, e^{j 2 \pi f \Delta}, \ldots, e^{j 2 \pi f(L-1) \Delta}\right]^{T} .
$$

For uniform linear arrays and $m$ known correlation lags, the maximurentropy method (MEM) is equivalent to order $m$ auto-regressive $(\operatorname{AR}(m))$ moll fitting and Linear Prediction where the extremal problem

$$
\min _{\mathbf{w}} E\left[\left\|\mathbf{w}^{B} \mathbf{y}(t)\right\|^{2}\right] \quad \text { subject to } \mathbf{w}^{H} \mathbf{u}_{1}=1,
$$

has as its solution the optimal $w$ given by

$$
\mathbf{w}=\left(\mathbf{u}_{1}^{H} \mathbf{R}^{-1} \mathbf{u}_{1}\right)^{-1} \mathbf{R}^{-1} \mathbf{u}_{1},
$$

which leads to a power spectrum identical to (2.11). To complete the MU formulation, the DOA estimates are associated with the minima of the null-spitrum (or maxima of inverse null-spectrum)

$$
P_{M E}(\theta)=\left\|\mathbf{a}(\theta)^{H} \mathbf{w}\right\|
$$

\subsubsection{Minimum Variance Method}

In 1969, Capon [21] argued that, in a filtering interpretation, the caventional beamforming at a given angle gives equal weight to all angles, so that the undesirable contribution from the other sources is not rejected. He propos a linear estimator which minimizes the interference from angles outside the regin of interest.

$$
\min _{\mathbf{w}} E\left[\left\|\mathbf{w}^{H} \mathbf{y}(t)\right\|^{2}\right] \text { subject to } \mathbf{w}^{H} \mathbf{a}(\theta)=1,
$$

The optimal solution is easily found using the standard Lagrange mulolier techniques. Minimizing

$$
J=\mathbf{w}^{H} \mathbf{R} \mathbf{w}+\lambda\left(\mathbf{w}^{H} \mathbf{a}(\theta)-1\right)
$$

with respect to $w$ yields

$$
\mathbf{w}=-\lambda \mathbf{R}^{-1} \mathbf{a}(\theta)
$$

where

$$
\lambda=\frac{1}{\mathbf{a}(\theta)^{H} \mathbf{R}^{-1} \mathbf{a}(\theta)}
$$


and the DOA estimates are associated with the minima of the null-spectrum (or maxima of inverse null-spectrum)

$$
P_{M V}(\theta)=\mathbf{a}(\theta)^{H} \mathbf{R}^{-1} \mathbf{a}(\theta) .
$$

\subsection{Subspace Based Algorithms}

The subspace approach to direction-of-arrival (DOA) estimation has found prominence because of its higher resolution and lower computation compared with other classical methods. It was pioneered by the work of Pisarenko [22].

\subsubsection{Subspace Decomposition}

The subspace decomposition can be performed on either the direct data matrix by a singular value decomposition (SVD), or on the covariance matrix by an eigenvalue decomposition. These two approaches are essentially equivalent are discussed in Chapter 3. For simplicity, we will use direct data matrix approach.

The singular value decomposition of a data matrix $\mathbf{Y}(L \times M)$ is

$$
\mathbf{Y}=\left(\begin{array}{ll}
\mathrm{U}_{3} & \mathrm{U}_{0}
\end{array}\right)\left(\begin{array}{cc}
\mathbf{\Sigma}, & 0 \\
0 & 0
\end{array}\right)\left(\begin{array}{c}
\mathrm{V}_{0}^{H} \\
\mathrm{~V}_{0}^{H}
\end{array}\right)=\mathbf{A}(\theta)^{B} \mathbf{S}
$$

where the diagonal elements of $\boldsymbol{\Sigma}$, are arranged as singular values in decreasing order.

$$
\sigma_{1} \geq \ldots \geq \sigma_{P}
$$

and for the noisy data $\tilde{\mathbf{Y}}$

$$
\tilde{\mathbf{Y}}=\left(\begin{array}{ll}
\tilde{\mathbf{U}}_{0} & \tilde{\mathbf{U}}_{0}
\end{array}\right)\left(\begin{array}{cc}
\tilde{\boldsymbol{\Sigma}}_{0} & 0 \\
0 & \tilde{\boldsymbol{\Sigma}}_{0}
\end{array}\right)\left(\begin{array}{c}
\tilde{\mathbf{V}}_{0}^{H} \\
\tilde{\mathbf{V}}_{0}^{H}
\end{array}\right),
$$

with

$$
\tilde{\sigma}_{1} \geq \ldots \geq \tilde{\sigma}_{P} \geq \tilde{\sigma}_{P+1} \geq \ldots \geq \tilde{\sigma}_{L} .
$$

The largest $P$ singular values correspond to the signal powers (plus noise for noisy case), and the $L-P$ smallest singular values (zero for noise-free case, close to 
$\sigma_{n}$ for noisy case) are corresponding to the noise power. Therefore the eigenspace spanned by singular vectors is divided into two orthonormal subspaces: the signal subspace spanned by singular vectors associated with largest $P$ singular values and the orthogonal subspace spanned by singular vectors associated with $L-P$ smallest singular values.

\subsubsection{MUSIC}

The MUSIC $[23,24,25]$ algorithm first estimates the signal subspace from array measurements, then estimates the parameters from the intersections between the array manifold and the estimated signal subspace. This search is typically carried out by computing a weighted Hermitian norm using the direction vectors for each angle of interest and kernel obtained from orthogonal singular vectors of the data matrix. It employs the fact that for signal directions $\theta_{k}, k=1, \cdots, P$,

$$
\mathbf{a}\left(\theta_{k}\right)^{H} \mathbf{U}_{0}=\mathbf{0} .
$$

Thus the DOA estimates of the MUSIC algorithm are given by the $P$ zeros of the null-spectrum

$$
P_{M U}(\theta)=\left\|\mathrm{a}(\theta)^{H} \mathrm{U}_{0}\right\|^{2} .
$$

In this and future equations, the symbol $\theta$ without a subscript is a scalar variable which represents a possible direction of arrival, while the subscripted symbol $\theta_{k}, k=1, \cdots, P$ refers to the actual directions of arrival in the noise-free data. The advantage of MUSIC is that no constraint on the geometry of the sensor array is required. The array pattern can be absolutely arbitrary as long as it is known or calibrated. Though performance advantages are substantial, they are achieved at a considerable cost in computation (search over parameter space) and storage (of array calibration data). Up to now MUSIC is the most popular and often cited work. It has been widely studied and new versions have been developed such as Weighted-MUSIC [26], and Root-MUSIC [1]. 


\subsubsection{Minimum-Norm}

The Min-Norm method $[\mathbf{2 7}, 28]$ is to apply the linear prediction method to uniform line array data. The first step is to identify a single vector $d$, with first element equal to unity and minimum Euclidian norm, in the span of the orthogonal subspace. The polynomial $D(z)$ with coefficient vector $\mathrm{d}$ has $L-1$ roots. The $P$ roots on the unit circle contain the DOA information and the $L-P-1$ extraneous roots tend to be uniformly distributed within unit circle.

If we partition the signal and orthogonal subspace vectors as follows

$$
\mathrm{U}_{\mathrm{s}}=\left(\begin{array}{l}
\mathrm{g}^{H} \\
\mathrm{U}_{0}^{\prime}
\end{array}\right) \text { or } \mathrm{U}_{0}=\left(\begin{array}{l}
\mathbf{c}^{H} \\
\mathrm{U}_{0}^{\prime}
\end{array}\right)
$$

where $g^{H}$ and $c^{B}$ are the first rows of $\mathrm{U}_{0}$ and $\mathrm{U}_{o}$, respectively, then from the orthogonality of $\mathrm{U}_{s}$ and $\mathrm{U}_{0}$,

$$
\mathrm{U}^{H} \mathrm{~d}=0 .
$$

the vector $d$ is solved from $(\mathbf{2 . 2 4})$ as

$$
\mathbf{d}=\left(\begin{array}{c}
1 \\
\frac{\mathrm{U}_{0}^{\prime} g}{\left.1-\|\|^{2}\right)}
\end{array}\right) \quad \text { or } \quad \mathrm{d}=\left(\begin{array}{c}
1 \\
\frac{\mathrm{U}_{0}^{\prime} \mathrm{c}}{\|c\|^{2}}
\end{array}\right) \text {. }
$$

This prediction-error vector $d$ is a linear combination of all the vectors in $U_{0}$ as we can see from

$$
\mathbf{d}=\mathbf{U}_{0} \frac{\mathbf{c}}{\|\mathbf{c}\|^{2}} \stackrel{\text { def }}{=} \mathrm{U}_{0} \mathbf{c} .
$$

To obtain DOA estimates, a polynomial rooting is then performed, i.e.,

$$
D(z)=\mathrm{a}(z)^{H} \mathrm{~d}=\prod_{i=1}^{L-1}\left(1-r_{i} z^{-1}\right) .
$$

In the case of noisy data, the $P$ roots which are closest to the unit circle are chosen as the signal-roots and the rest are regarded as noise roots. There are other more elaborate signal-root selection procedures. The directions of arrival are found from the angles of signal roots.

The Min-Norm method is also applicable to arbitrary array geometry by searching for the $P$ zeros of the null-spectrum over $\theta[17]$

$$
P_{M N}(\theta)=\left|a^{H}(\theta) \mathrm{d}\right|^{2} .
$$




\subsubsection{Root-MUSIC Algorithm}

As an improvement of MUSIC algorithms, Root-MUSIC, forms and roots the following null-spectrum polynomial [1]

$$
\begin{aligned}
P_{R M}(z) & =\mathrm{a}\left(z^{-1}\right)^{T} \mathrm{U}_{0} \mathrm{U}_{0}^{H} \mathrm{a}(z) \\
& =A \prod_{i=1}^{L-1}\left(1-r_{i} z^{-1}\right)\left(1-r_{i}^{*} z\right) .
\end{aligned}
$$

The polynomial $P_{R M}(z)$ has 2(L-1) roots. Unlike Min-Norm, Root-MUSIC always chooses the $P$ roots with largest amplitudes inside unit-circle. This choice results in a bias in the radial direction of the estimated roots since when white Gaussian observation noise is present the signal-roots will be perturbed inside and outside the unit-circle. However, DOA estimates are only functions of the angles of the roots, not the radii. Thus the radial bias does not affect the DOA estimates obtained by Root-MUSIC.

\subsubsection{State-Space Realization}

The state space approach includes a covariance approach and a direct-data approach $[29,30,31,32]$. When auto-correlation estimates are used, the covariance matrix is Toeplitz (yielding the TAM algorithm) but this matrix does not have the low-rank property even with noise-free data; for the case of using covariance estimates $\left(R=\frac{1}{M} \mathbf{Y}^{H} \mathbf{Y}\right)$, the covariance approach is equivalent to direct-data approach (sometimes called DDA).

The State-Space approach can be used with an ESPRIT-type array (see next subsection), but what follows is based on a uniform line array for the purpose of analysis.

Recall

$$
\begin{aligned}
y_{i}(t) & =\sum_{k=1}^{P} e^{j \frac{2 \pi d}{\lambda_{e}} \sin \theta_{k}(i-1)} s_{k}(t) \\
& =\left[e^{j \frac{2 \pi d}{\lambda_{c}} \sin \theta_{1}(i-1)}, \ldots, e^{j \frac{2 \pi d}{\lambda_{c}} \sin \theta_{P}(i-1)}\right] \mathrm{s}(t) .
\end{aligned}
$$


A state-space model for a plane-wave signal propagating in a sensor array can be derived as

$$
\begin{aligned}
\mathbf{x}_{i+1}(t) & =\mathbf{F} \mathbf{x}_{i}(t) \\
y_{i}(t) & =\mathbf{b} \mathbf{x}_{i}(t)
\end{aligned}
$$

or

$$
y_{i}(t)=\mathbf{h} \mathbf{F}^{i-1} \mathbf{x}_{1}(t)
$$

where the $x$ is the state-vector with the initial value $x_{1}(t)=s(t)$ and $h=[1, \cdots, 1]$ is row-vector of sensor gains, and

$$
\mathbf{F}=\left(\begin{array}{cccc}
e^{j \frac{2 \pi d}{\lambda_{c}} \sin \theta_{1}} & 0 & \cdots & 0 \\
0 & e^{j \frac{2 \pi d}{\lambda_{c}} \sin \theta_{2}} & \ddots & \vdots \\
\vdots & \ddots & \ddots & 0 \\
0 & \cdots & 0 & e^{j \frac{2 \pi d}{\lambda_{c}} \sin \theta_{p}}
\end{array}\right) .
$$

The diagonal entries of $\mathbf{F}$ in a diagonal realization (in general, eigenvalues of the system matrix in an arbitrary realization) contain the information of arrival direction. The data matrix is formed and factored as follows

$$
\begin{aligned}
\mathbf{Y} & =\left(\begin{array}{ccc}
y_{1}(1) & \ldots & y_{1}(M) \\
\vdots & \ddots & \vdots \\
y_{L}(1) & \ldots & y_{L}(M)
\end{array}\right) \\
& =\left(\begin{array}{c}
\mathbf{h} \\
\mathbf{h F} \\
\vdots \\
\mathbf{h} \mathbf{F}^{L-1}
\end{array}\right)\left(\begin{array}{lll}
\mathbf{s}(0) & \ldots & \mathbf{s}(M)) \stackrel{\text { def }}{=} \text { OC. }
\end{array}\right.
\end{aligned}
$$

$\mathbf{O}$ and $\mathbf{C}$ are respectively the observability matrix and controllability matrix. Let $O^{\dagger}$ be $O$ except the first row and $O^{\dagger}$ be $O$ except the last row. From (2.31) it is clear that the following shift-invariance is true

$$
\mathbf{O}^{\dagger} \mathbf{F}=\mathbf{O}^{\downarrow}
$$


Therefore a transition matrix $\mathbf{F}$ is obtained from (2.32)

$$
\mathbf{F}=\mathbf{O}^{\dagger} \mathbf{O}^{\downarrow}
$$

In general, the factors of the data matrix are obtained from a singular value decomposition (2.22). By taking the principal components and using the concept of state-variable balancing

$$
\mathbf{O}=\mathbf{U}, \boldsymbol{\Sigma}^{\frac{1}{3}}
$$

and then $\mathbf{F}$ can be solved for as

$$
\mathbf{F}=\mathbf{O}^{\dagger} \mathbf{O}^{\dagger}
$$

The eigenvalues of $\mathbf{F}$ provide the estimates of arrival direction.

\subsubsection{ESPRIT}

Estimation of Signal Parameters via Rotational Invariant Techniques (ESPRIT) $[33,34,35,36,37,38,39,40,41]$ mitigates the computational and storage requirements of MUSIC by exploiting an underlying displacement invariance structure of an array of sensors. In order to do this, another array which is a displaced version of the first array is needed. This could be implemented by one array with pairwise matched sensor doublets. To describe mathematically the effect of the shift-invariance of the sensor array, it is convenient to describe the array as being comprised of two subarrays identical in every respect although physically displaced from each other by a known displacement quantity $\Delta$. The signals received at each subarray are

$$
\begin{aligned}
z(t) & =\operatorname{As}(t) \\
\mathbf{x}(t) & =\operatorname{AFs}(t)
\end{aligned}
$$


The matrix $\mathbf{F}$ is diagonal $P \times P$ matrix of the phase delays between the subarrays for the $P$ wavefronts and is given by

$$
\mathbf{F}=\left(\begin{array}{cccc}
e^{j \frac{2 \pi \Delta}{\lambda_{\theta}} \sin \theta_{1}} & 0 & \cdots & 0 \\
0 & e^{j \frac{j \pi \Delta}{\lambda_{\theta}} \sin \theta_{3}} & \ddots & \vdots \\
\vdots & \ddots & \ddots & 0 \\
0 & \cdots & 0 & e^{j \frac{2 \pi \Delta}{\lambda_{\theta}} \sin \theta_{P}}
\end{array}\right)
$$

Note that $\mathbf{F}$ is a unitary matrix (operator) that relates the measurements from subarray $z$ and those from $x$. Now define

$$
y(t)=\left(\begin{array}{c}
z(t) \\
x(t)
\end{array}\right)=\left(\begin{array}{c}
\mathbf{A} \\
\text { AF }
\end{array}\right) s(t) .
$$

Using SVD

$$
\mathbf{Y}=\mathbf{U} \mathbf{\Sigma V}^{H}=\left(\begin{array}{c}
\mathrm{U}_{x} \\
\mathbf{U}_{\varepsilon}
\end{array}\right) \mathbf{\Sigma V}^{H} .
$$

A matrix-pencil is formed as

$$
\mathbf{U}_{s x} \mathbf{F}=\mathbf{U}_{e x}
$$

where $U_{s z}\left(U_{s z}\right)$ are the principal components from $U_{z}\left(U_{z}\right)$. If we pre-multiply (2.38) by a full-rank $(P \times L) \mathrm{T}^{H}$, then the generalized eigenvalues of the matrixpencil in (2.38) are equal to the eigenvalues of

$$
\mathbf{F}=\left(\mathbf{T}^{H} \mathbf{U}_{\boldsymbol{\theta z}}\right)^{-\mathbf{1}} \mathbf{T}^{H} \mathbf{U}_{\boldsymbol{\theta z}} .
$$

Since we use a uniform line array here to simplify analysis, the best selection of $U_{s z}$ and $U_{s z}$ are the first $L-1$ and the last $L-1$ rows of $U_{s}$, respectively. Then $\Delta=d$. If we choose $\mathbf{T}=\mathbf{U}_{\theta s}$, the ESPRIT method is equivalent to State-Space Realization $[42,43]$. Here, we choose $\mathbf{T}=\mathbf{U}_{s z}$ as in [39] to yield

$$
\mathbf{F}=\left(\mathbf{U}_{s x}^{H} \mathbf{U}_{s x}\right)^{-1} \mathbf{U}_{s x}^{H} \mathbf{U}_{s x} .
$$

ESPRIT has certain advantages over MUSIC:

- It does not require knowledge of the array geometry and element characteristics. 
- It is computationally much less complex.

However, there are still some strong constraints of array geometry: the corresponding sensors in each subarray must have identical characteristics, and they must be equally displaced, in parallel and in the same plane.

\subsubsection{Matrix-Pencil Method}

The ideas that the Matrix-Pencil Method $[44,45,46]$ and ESPRIT method exploit are identical despite the fact that each of them has many different versions. ESPRIT was proposed from an array geometrical design point of view while the Matrix-Pencil method was developed in applying results from linear algebra to the problem of direction-of-arrival estimation. In this sense, we can say that the Matrix-Pencil Method is a generalized version of State-Space Realization and ESPRIT. The Matrix-Pencil Method forms two matrices with certain relationship in between, then solves the generalized eigenvalue problem of matrix-pencil by employing that relation. Three versions of the Matrix-Pencil Method summarized in [47] are as follows:

1. A matrix pencil is

$$
\mathbf{x}-\lambda \mathbf{z}
$$

where $\lambda$ can be solved from a generalized eigenvalue problem

$$
\left(\mathbf{z}^{\dagger} \mathbf{X}-\lambda_{k}\right) \mathbf{q}_{k}=0
$$

2. Form a data matrix

$$
\mathbf{Y}=\left(\begin{array}{c}
\mathbf{X} \\
\mathbf{z}
\end{array}\right)
$$

and take the principal components from the singular value decomposition to the same data (2.38) using ESPRIT notation. A matrix pencil is then formed as

$$
\mathbf{U}_{s \varepsilon}-\lambda \mathbf{U}_{\bullet \varepsilon}
$$


and $\lambda$ is solved as an eigenvalue of $F$ in (2.38). This solution is from signal subspace invariance as in ESPRIT (for this reason, we combine the analysis of the Matrix-Pencil method into the analysis ESPRIT).

3. A total least-squares approach [47] (which is also applicable to State-Space Realization and ESPRIT).

\subsubsection{Remark}

Many researchers have been making efforts to unify these subspace processing algorithms. In the linear equispaced array case, there are very clear connections among these methods. The null-spectrum of MUSIC is calculated from the intersection between the array manifold and all the orthogonal vectors, while the null-spectrum of Min-Norm is from the intersection between the array manifold and a single min-norm vector from the orthogonal subspace. The subarray selection of ESPRIT is equivalent to State-Space Realization. ESPRIT, Matrix-Pencil and State-Space Realization use essentially the same shift-invariance property of the signal subspace. The MUSIC and Min-Norm searching algorithms are valid for arbitrary array geometry, while ESPRIT, Matrix-Pencil and State-Space Realization require the certain geometric constraints to obtain the desired shift-invariance properties. The polynomial rooting approach is only applicable to the case of a linear equispaced sensor array. Comparative studies of some of above methods can be found in $[48,42,43,49,50]$.

\subsection{Classification of The Subspace Algorithms}

\subsubsection{Classification by Subspace}

The subpace-based algorithms can be classified into two categories by the subspace which an individual algorithm utilizes:

- Orthogonal-Subspace Algorithms: Pisarenko Method, MUSIC (including Root-MUSIC) and Min-Norm (including searching algorithm). 
- Signal-Subspace Algorithms: State-Space Realization (TAM and DDA), ESPRIT and Matrix-Pencil method.

In the light of the above classification, the analysis therefore starts with the perturbation of each subspace in Chapter 3.

\subsubsection{Classification by Numerical Procedure}

The subspace-based algorithms can also be classified in to three approaches by the numerical procedure which an individual algorithm exploits:

- Extrema-Searching Approach: MUSIC and Min-Norm searching algorithm.

- Polynomial-Rooting Approach: Pisarenko, Min-Norm and Root-MUSIC.

- Matrix-Shifting Approach: State-Space Realization, ESPRIT and MatrixPencil method.

We will establish a common model for each approach, analyze the common model and specialize the results to each algorithm in Chapters 4, 5, and 6. 


\section{Chapter 3}

\section{Perturbation of Subspaces}

\subsection{Introduction}

All the subspace processing algorithms utilize the properties of subspaces obtained by subspace decomposition. To analyze the different subspace processing algorithms, it is important to analyze the subspaces. It not only makes the analysis better founded, but also provides a common basis for performance comparison of these algorithms. The direction of arrival estimates can be expressed as functions of the estimated subspace. Thus to compute the perturbations in the estimated directions of arrival, we need to to compute the perturbations in the estimated subspaces. Most existing analyses focus on parameters of interest such as directions of arrival or intermediate parameters like prediction coefficients, and not on the underlying subspaces. As a result, they fail to give a unified analysis. Our analysis originated from examining the perturbation of the linear prediction error vector [17], and then examining the perturbations of the orthogonal subspace [51] and of the signal-subspace [52].

The effects of perturbations on an estimation algorithm can be classified as "above threshold" or "below threshold". An algorithm is said to be above threshold when the mean-squared error (MSE) of the parameters is close to the CramerRao Bound. On the other hand, when the MSE is much larger than the C-R bound, the algorithm is said to be below threshold. The threshold phenomenon is 
a characteristic of nonlinear estimation algorithms. A threshold analysis of MinNorm algorithm is given in [53]. In this work, we assume that the SNR is high enough so that the algorithm is not in threshold.

\subsection{Subspace Review}

Assume there are $P$ uncorrelated plane waves simultaneously incident on an $L$ sensor array. Take $M$ snapshot simultaneously at each sensor. The data matrix is formed as

$$
\begin{aligned}
\mathbf{Y} & =\left(\begin{array}{ccc}
\mathbf{y}(1) & \ldots & \mathbf{y}(M)
\end{array}\right) \\
& =\left(\begin{array}{ccc}
y_{1}(1) & \ldots & y_{1}(M) \\
\vdots & \ddots & \vdots \\
y_{L}(M) & \ldots & y_{L}(M)
\end{array}\right) \\
& =\mathbf{A}(\theta) \mathbf{S}
\end{aligned}
$$

where the subscript is spatial index of the sensors and the index in parenthesis is temporal for snapshots. The covariance estimate of the data matrix and its eigenvalue decomposition are

$$
\begin{aligned}
\mathbf{R} & =\frac{1}{M} \mathbf{Y} \mathbf{Y}^{H} \\
& =\left(\begin{array}{ll}
\mathbf{E}, & \mathbf{E}_{o}
\end{array}\right)\left(\begin{array}{cc}
\mathbf{\Lambda} & 0 \\
0 & 0
\end{array}\right)\left(\begin{array}{l}
\mathbf{E}_{。}^{H} \\
\mathbf{E}_{o}^{H}
\end{array}\right) \\
& =\mathbf{A}(\theta) \mathbf{R}, \mathbf{A}(\theta)^{H}
\end{aligned}
$$

where $\mathbf{E}_{\boldsymbol{g}}$ are the eigenvectors associated with $\boldsymbol{P}$ non-zero eigenvalues, which span the signal subspace, while $\mathbf{E}_{o}$ are the eigenvectors associated with the zero eigenvalues, which define the orthogonal subspace. The term "noise-subspace" has been previously used to describe what we call the orthogonal subspace. Since this subspace is the orthogonal complement of the signal-subspace, and since it is well 
defined whether or not any noise is present in the problem, we believe the term "orthogonal-subspace" is more appropriate.

There are two important properties of the subspaces which the subspace based algorithms exploit:

- The vectors in $\mathbf{E}$, span the signal subspace which is identical to the array manifold (column space of $\mathbf{A}(\theta)$ ).

- The vectors in $\mathbf{E}_{0}$ span the orthogonal subspace which is orthogonal to the array manifold. This orthogonality can be expressed as

$$
\mathrm{a}\left(\theta_{k}\right)^{H} \mathbf{E}_{0}=0, \quad k=1, \ldots, P \text {. }
$$

The above result can be seen from the fact that

$$
\mathbf{E}_{0}^{H} \mathbf{R}=0
$$

since

$$
\mathbf{E}_{0}^{H} \mathbf{E} s=0
$$

Even with presence of additive observation noise,

$$
\tilde{\mathbf{Y}}=\mathbf{Y}+\mathbf{N}
$$

and

$$
\tilde{\mathbf{R}}=\frac{1}{M} \tilde{\mathbf{Y}} \tilde{\mathbf{Y}}^{H}=\left(\begin{array}{ll}
\tilde{\mathbf{E}}_{s} & \tilde{\mathbf{E}}_{0}
\end{array}\right)\left(\begin{array}{cc}
\tilde{\mathbf{\Lambda}}_{s} & 0 \\
0 & \tilde{\boldsymbol{\Lambda}}_{0}
\end{array}\right)\left(\begin{array}{c}
\tilde{\mathbf{E}}_{s}^{H} \\
\tilde{\mathbf{E}}_{0}^{H}
\end{array}\right)
$$

under the ergodic assumption,

$$
\lim _{M \rightarrow \infty} \tilde{\mathbf{R}}=\mathbf{A}(\theta) \mathbf{R}, \mathbf{A}(\theta)^{H}+\sigma_{n}^{2} \mathbf{I}
$$

the orthogonal-subspace is still (asymptotically) orthogonal to the signal manifold. A simple proof can be stated as follows:

For $i>P, \lambda_{i}=\sigma_{n}^{2}$, and

$$
\begin{aligned}
\mathbf{A}(\theta) \mathbf{R}_{s} \mathbf{A}(\theta)^{H} \mathbf{e}_{i} & =\left(\mathbf{R}-\sigma_{n}^{2} \mathbf{I}\right) \mathbf{e}_{i} \\
& =\left(\lambda_{i}-\sigma_{n}^{2}\right) \mathbf{I} \mathbf{e}_{i} \\
& =0
\end{aligned}
$$


where $e_{i}$ is the eigenvector of $\mathbf{R}$ associated with eigenvalue $\lambda_{i}$. Since $\mathbf{A}(\theta) \mathbf{R},(L \times P)$ has rank of $P$, there is a $P \times L$ matrix $\mathrm{D}$ of rank $P$ which satisfies

$$
\mathbf{D A}(\theta) \mathbf{R},=\mathbf{I}_{d}
$$

Then

$$
\begin{aligned}
\mathbf{D A}(\theta) \mathbf{R}_{d} \mathbf{A}(\theta)^{H} \mathbf{e}_{i} & =\mathbf{I}_{d} \mathbf{A}(\theta)^{H} \mathbf{e}_{i} \\
& =\mathbf{A}(\theta)^{H} \mathbf{e}_{i} \\
& =0
\end{aligned}
$$

this is equivalent to (3.3).

The subspace decomposition can also be performed on the direct-data matrix $\mathbf{Y}$ shown in (3.1) by a singular value decomposition. Let the SVD of $\mathbf{Y}$ be denoted as

$$
\mathbf{Y}=\mathbf{U} \mathbf{\Sigma} \mathbf{V}^{H}=\left(\begin{array}{ll}
\mathrm{U}, & \mathrm{U}_{0}
\end{array}\right)\left(\begin{array}{cc}
\mathbf{\Sigma}, & 0 \\
0 & 0
\end{array}\right)\left(\begin{array}{c}
\mathbf{V}_{0}^{H} \\
\mathbf{V}_{0}^{H}
\end{array}\right) .
$$

Then we get the same results as before:

- The vectors in $U$, span the signal subspace which is identical to array manifold (column space of $\mathbf{A}(\theta)$ ).

- The vectors in $U_{o}$ span the orthogonal subspace which is orthogonal to the array manifold. This orthogonality can be expressed as $\mathbf{a}\left(\theta_{k}\right)^{H} \mathbf{U}_{o}=0$, for $k=$ $1, \ldots, P$.

In a noisy environment,

$$
\tilde{\mathbf{Y}}=\tilde{\mathbf{U}} \tilde{\mathbf{\Sigma}} \tilde{\mathbf{V}}^{H}=\left(\begin{array}{ll}
\tilde{\mathbf{U}}, & \tilde{\mathbf{U}}_{0}
\end{array}\right)\left(\begin{array}{cc}
\tilde{\mathbf{\Sigma}}_{t} & 0 \\
0 & \tilde{\mathbf{\Sigma}}_{0}
\end{array}\right)\left(\begin{array}{c}
\tilde{\mathbf{V}}_{t}^{H} \\
\tilde{\mathbf{V}}_{0}^{H}
\end{array}\right) .
$$

Obviously, we can see

$$
\boldsymbol{\Sigma}^{2}=\mathbf{\Lambda}, \quad \mathbf{U}=\mathbf{E}
$$

and

$$
\tilde{\boldsymbol{\Sigma}}^{2}=\tilde{\mathbf{\Lambda}} M, \quad \tilde{\mathbf{U}}=\tilde{\mathbf{E}} .
$$


We assume that the singular vectors are normalized so that $\mathbf{U}^{H} \mathbf{U}=\mathbf{I}$ and $\tilde{\mathbf{U}}^{H} \tilde{\mathbf{U}}=$ I.

As shown above the subspaces obtained from the eigenvalue decomposition of a covariance matrix and the subspaces from a singular value decomposition of the direct data matrix are the same. In this work, all the algorithms used will be based on the direct-data. They will have the same performance as the covariance-based algorithm described above, with perhaps slightly better numerical properties. One reason that the direct-data algorithm might not be often used in practice is that the dimensions of the matrices grow with the data length (number of observations), while the covariance matrix has fixed dimensions (number of sensors). However, for the purpose of analysis, the direct-data algorithm is much easier to deal with. Because we do not restrict our analysis on uniform line array geometry, only the forward data formulation will be considered in this chapter.

\subsection{Perturbation of Orthogonal-Subspace}

Recall that the columns of $U_{0}$ are an arbitrary orthonormal basis for the orthogonal-subspace of the noise-free signal matrix $\mathbf{Y}$, and the columns of $\tilde{\mathbf{U}}_{o}$ are the estimated orthogonal-subspace vectors associated with smallest singular values $\tilde{\boldsymbol{\Sigma}}$ of the noisy data matrix $\tilde{\mathbf{Y}}$. We can write

$$
\tilde{\mathbf{U}}_{0}=\mathbf{U}_{0}+\Delta \mathbf{U}_{0}
$$

where $\Delta \mathbf{U}_{o}$ is the perturbation in the estimated orthogonal-subspace vectors. The additive noise in the data matrix $\tilde{\mathbf{Y}}=\mathbf{Y}+\mathbf{N}$ is transformed in a highly nonlinear way by the SVD to produce the noisy singular vectors $\tilde{U}_{0}[54,55]$. It is noted that the perturbations in estimated subspaces which produce errors in DOA estimates are those orthogonal to the original noise-free subspace under consideration. For orthogonal subpace-based algorithms, only the signal-subspace component of the perturbation in estimated orthogonal subspace is relevant; for signal-subspace based algorithms, only the orthogonal-subspace component of the perturbation in the estimated signal-subspace is relevant. At high SNR, we seek an expression for 
$\Delta \mathbf{U}_{0}$ which is linear in the noise matrix $\mathbf{N}$ and which is the projection of $\tilde{\mathbf{U}}_{0}$ onto U. Such an expression can be found by a forward error analysis using a Taylor series expansion for the SVD. This approach has been used for a related problem and was found that the resulting expression was extremely cumbersome [56]. In our previous derivation, we started with a minimization problem for which $\tilde{U}_{0}$ is the solution and by approximating this problem, we obtained a simple expression for $\Delta \mathbf{U}_{o}$. This so-called backward error analysis was first used in [16] to develop a performance analysis for linear prediction algorithms suggested by Tufts. It has been found that forward and backward error analyses give about the same performance predictions, but the expressions resulting from the backward analysis are much simpler than those resulting from the forward analysis.

In this section, we give a simple derivation of $\Delta U_{0}$ based on perturbation expansion theory suggested by Dr. G. W. Stewart. This derivation relies on the assumption of high SNR (which all other perturbation analyses assume either implicitly or explicitly).

Let us seek $\Delta \mathbf{U}_{\text {o }}$ in the form of $\mathbf{U}, \mathbf{P}$, where $\mathbf{P}$ is of order of the noise $\mathbf{N}$ (similarly define $\Delta \mathrm{V}_{o}=\mathbf{V}, \overline{\mathbf{P}}$ ). Then pre-multiply $\overline{\mathbf{Y}}=\tilde{\mathbf{U}} \tilde{\boldsymbol{\Sigma}} \tilde{\mathbf{V}}^{H}$ by $\tilde{\mathrm{U}}_{0}^{H}$ to obtain

$$
\tilde{\mathbf{U}}_{0}^{H} \tilde{\mathbf{Y}}=\tilde{\mathbf{\Sigma}}_{0} \tilde{\mathbf{V}}_{o}^{H} .
$$

Using the fact that $\tilde{\Sigma}_{o}=\Delta \Sigma_{o},(3.9)$ can be written as

$$
\left(\mathbf{U}_{0}+\Delta \mathbf{U}_{0}\right)^{H}(\mathbf{Y}+\mathbf{N})=\Delta \Sigma_{0}\left(\mathbf{V}_{0}+\Delta \mathbf{V}_{0}\right)^{H}
$$

Substituting $\Delta \mathbf{U}_{0}=\mathbf{U}_{\mathbf{s}} \mathbf{P}$ and $\Delta \mathbf{V}_{0}=\mathbf{V}_{\mathbf{s}} \overline{\mathbf{P}}$ into (3.10), we get

$$
\left(\mathbf{U}_{0}+\mathbf{U}, \mathbf{P}\right)^{H}(\mathbf{Y}+\mathbf{N})=\Delta \Sigma_{0}\left(\mathbf{V}_{0}+\mathbf{V}, \mathbf{P}\right)^{H} .
$$

By neglecting second order terms and using the fact that $\mathbf{U}_{o}^{H} \mathbf{Y}=0$, we get

$$
\mathbf{U}_{0} \mathbf{N}+\mathbf{P}^{H} \mathbf{U}_{s}^{H} \mathbf{Y}=\Delta \boldsymbol{\Sigma}_{0} \mathbf{V}_{0}^{H} .
$$

Finally, we post-multiply (3.12) by $\mathbf{V}$, and simplify to obtain

$$
\mathbf{U}_{o}^{H} \mathbf{N V},+\mathbf{P}^{H} \mathbf{\Sigma},=0
$$


We can solve for $\mathbf{P}$ from (3.13) as follows

$$
\mathbf{P}=-\mathbf{\Sigma}_{。}^{-1} V_{,}^{H} \mathbf{N}^{H} \mathbf{U}_{0}
$$

and then we have

$$
\Delta \mathbf{U}_{0}=-\mathbf{U}_{0} \mathbf{\Sigma}_{0}^{-1} V_{0}^{H} \mathbf{N}^{H} U_{0} .
$$

Note that (3.15) expresses $\Delta U_{o}$ as a linear function of the noise matrix.

We emphasize the fact that (3.15) is a general expression for the perturbation of the orthogonal-subspace due to additive noise in the data matrix. This expression can be used to analyze the performance of any algorithm which estimates the orthogonal-subspace from data.

\subsection{Perturbation of Signal-Subspace}

We start the investigation of the signal-subspace perturbation by assuming $\tilde{U}_{0}=$

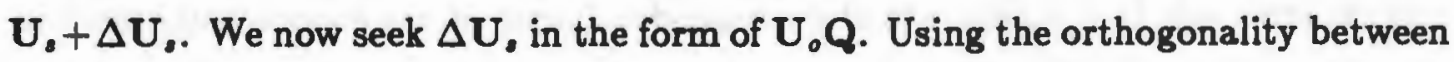
the the orthogonal- and signal- subspaces, we have

$$
\tilde{\mathbf{U}}_{0}^{H} \tilde{\mathbf{U}}_{,}=0 \text {. }
$$

Equivalently, we have

$$
\left(\mathbf{U}_{0}+\Delta \mathbf{U}_{0}\right)^{H}\left(\mathbf{U}_{0}+\Delta \mathbf{U}_{0}\right)=0
$$

Substitute $\Delta \mathbf{U}_{0}=\mathbf{U}, \mathbf{P}$ and $\Delta \mathbf{U}_{0}=\mathbf{U}_{0} \mathbf{Q}$ into (3.17),

$$
\left(\mathbf{U}_{0}+\mathbf{U}, \mathbf{P}\right)^{B}\left(\mathbf{U}_{\diamond}+\mathbf{U}_{0} \mathbf{Q}\right)=0
$$

or

$$
\mathbf{U}_{0}^{H} \mathbf{U}_{0}+\mathbf{U}_{0}^{H} \mathbf{U}_{0} \mathbf{Q}+\mathbf{P}^{H} \mathbf{U}_{0}^{H} \mathbf{U}_{0}+\mathbf{P}^{H} \mathbf{U}_{0}^{H} \mathbf{U}_{0} \mathbf{Q}=0 .
$$

Notice the noise-free signal- and orthogonal- subspaces are orthogonal, so the first term and the last term of above equation are zero. Since $\mathbf{U}_{0}^{H} \mathbf{U}_{o}=\mathbf{I}$ and $\mathrm{U}_{9}^{H} \mathrm{U}_{s}=\mathrm{I}$, we then have

$$
\mathbf{Q}=-\mathbf{P}^{H}=\mathbf{U}_{0}^{H} \mathbf{N V}, \boldsymbol{\Sigma}_{o}^{-1}
$$


and this gives us

$$
\Delta \mathbf{U}_{0}=\mathbf{U}_{0} \mathbf{U}_{0}^{H} \mathbf{N}, \mathbf{\Sigma}_{s}^{-1}
$$

Notice that in deriving the perturbation of signal-subspace, no further approximation was made, so the perturbation expansion for the signal-subspace is as good as the perturbation expansion for the orthogonal-subspace.

\subsection{Summary}

This chapter presents the perturbation analysis of subspaces obtained from a singular value decomposition of a data matrix formed using noisy data. The expressions for the perturbed subspaces are derived using perturbation theory. We first derived the orthogonal-subspace perturbation through a first-order perturbation expansion, then derived the signal-subspace perturbation using the orthogonality between the two subspaces. The columns of the perturbation matrix for the orthogonal-subspace are in signal-subspace while the columns of the perturbation matrix for signal-subspace are in the orthogonal-subspace. To first-order, the perturbation of subspaces is linear in the observation noise. The analysis of the subspace perturbation will provided a common ground for the comparison of various subspace processing algorithms. 


\section{Chapter 4}

\section{Performance of Extrema}

\section{Searching Algorithms}

\subsection{Introduction}

People had used the classical Fourier analysis and conventional beamforming to process sensor array signals and to estimate the directions of arrival until the late 1960's when Burg's Maximum-Entropy Methods [20] and Capon's MinimumVariance Method [21] were proposed in order to increase the resolution in array signal processing. In the 1970 's, a high-resolution approach - subspace processing emerged, pioneered by Pisarenko [22]. In the late 1970's, Schmidt $[23,24]$ and, independently, Bienvenu and Kopp [25] suggested the MUSIC algorithm which became a landmark of subspace processing. In the early 80's, another important method Minimum-Norm Linear Prediction was developed by Tufts and Kumaresan [27, 28]. Pisarenko's method, MUSIC and Min-Norm were the first three subspace processing methods. They all utilize the orthogonality between the signal manifold and orthogonal-subspace to find the directions of arrival. MUSIC and Min-Norm give much better performance than Pisarenko's method. MUSIC and Min-Norm have gained popularity because of their excellent performance and have been widely 
used and extensively cited. Also they have attracted most of performance analyses, $[2,57,4,58,14,15,11,12,13,59,51]$ for MUSIC and $[17,60,8]$ for Min-Norm searching algorithm.

Originally, Pisarenko's method and the Min-Norm method used the polynomial rooting procedure based on uniform line array geometry; while MUSIC used the extrema searching procedure based on arbitrary (calibrated) array geometry. Later, a new version of MUSIC - Root-MUSIC was developed by Barabell [1] and MinNorm Linear Prediction has been applied to arbitrary (calibrated) array geometry by Li, Vaccaro and Tufts [17]. A similar application of Pisarenko's method to arbitrary array geometry is easy to show [see Appendix B].

The major advantage of the searching algorithm is that array geometry is arbitrary as long as the sensor locations are calibrated, i.e. the sensor locations $\left(x_{i}, y_{i}\right)$ for $i=1, \ldots, L$ are known. The response of the $i$-th sensor to the signal arriving at the $k$-th angle $a_{i}\left(\theta_{k}\right)$ is

$$
a_{i}\left(\theta_{k}\right)=e^{j \frac{2 \pi}{\lambda_{a}}\left(x_{i} \sin \theta_{k}+y_{i} \cos \theta_{k}\right)} \text { for } i=1, \ldots, L ; k=1, \ldots, P .
$$

In this chapter, we analyze the searching algorithms of MUSIC and Min-Norm. Two versions of them - a MUSIC algorithm based on direct-data and a MinNorm algorithm for arbitrary array geometry will be used. A description of these algorithms can be found, respectively, in [17],[51] and [61]. Pisarenko's Method, because of using different data formulation, is analyzed in Appendix B.

\subsection{Common Model}

Now consider a common problem, which has been referred as Weighted MUSIC [26]. The algorithm consists of searching for the $P$ zeros over $\theta$ of the null-spectrum

$$
P(\theta)=\mathbf{a}(\theta)^{H} \mathbf{U}_{0} \mathbf{W} \mathbf{U}_{0}^{H} \mathbf{a}(\theta)
$$

where $\mathbf{W}$ is the weighting matrix and $\theta$ is a scalar variable which represents a possible direction of arrival. Then by (3.3)

$$
P\left(\theta_{k}, \mathbf{U}_{o}\right)=0 .
$$


Both MUSIC and Min-Norm can be considered as special cases of this algorithm by choosing the weights appropriately.

MUSIC: the weighting matrix is the identity matrix

$$
\mathbf{W}=\mathbf{I} \text {. }
$$

Min-Norm: weighting matrix is rank 1 and has the form of

$$
\mathbf{W}=\mathbf{c} \mathbf{c}^{H} \text {. }
$$

Equation (4.1) provides a common basis for our analysis of MUSIC and MinNorm searching algorithms.

\subsection{Perturbation of the Angle Estimates}

In a noisy environment, the estimated angles of arrival are denoted as perturbations from the true directions of arrival as

$$
\tilde{\theta}_{k}=\theta_{k}+\Delta \theta_{k}, \quad k=1, \ldots, P
$$

where the $\Delta \theta_{k}$ is the perturbation of the $k$-th direction of arrival. For the purpose of analysis, we adopt the following two steps in analyzing $\Delta \theta_{k}$ :

1. Derive the perturbation of the DOA's from $P\left(\theta, \tilde{U}_{0}\right)$ by one Newton step initialized at true arrival angle $\theta_{k}$.

2. Approximate the Newton Step by a linear function of the orthogonal-subspace perturbation $\Delta \mathrm{U}_{0}$.

Step 1. Perturbation of the DOA's from one Newton step of $P\left(\theta_{k}\right)$.

We assume at high SNR that the estimated angles can be found by one step of the Newton algorithm for finding the minima (no longer zeros) of the estimated nullspectrum function $P\left(\theta, \tilde{\mathbf{U}}_{o}\right)$ when the algorithm is initialized at a true direction, $\theta_{k}$. The Newton method attempts to find a direction $\tilde{\theta}_{k}$ which causes the first 
derivative of the null-spectrum function $P\left(\theta, \tilde{U}_{o}\right)$ to be zero. The resulting $\tilde{\theta}_{k}$ will then minimize $P\left(\theta, \tilde{\mathrm{U}}_{0}\right)$. This gives the following formula for the estimated angles

$$
\tilde{\theta}_{k}=\theta_{k}-\frac{\frac{\partial P\left(\theta_{k}, \check{\mathbf{U}}_{o}\right)}{\partial \theta}}{\frac{\partial^{2} P\left(\theta_{k}, \check{\mathrm{U}}_{o}\right)}{\partial \theta^{2}}}, \text { for } k=1, \cdots, P
$$

The first- and second- order partial derivatives of $P\left(\theta, \mathrm{U}_{0}\right)$ with respect to $\theta$ are computed as follows,

$$
\begin{aligned}
-\frac{\partial P\left(\theta, \mathbf{U}_{0}\right)}{\partial \theta} & \stackrel{\text { def }}{=} N\left(\theta, \mathbf{U}_{0}\right) \\
& =-\mathbf{a}^{(1)}(\theta)^{H} \mathbf{U}_{0} \mathbf{W} \mathbf{U}_{0}^{H} \mathbf{a}(\theta)-\mathbf{a}(\theta)^{H} \mathbf{U}_{0} \mathbf{W} \mathbf{U}_{0}^{H} \mathbf{a}^{(1)}(\theta)
\end{aligned}
$$

and

$$
\begin{aligned}
& \frac{\partial^{2} P\left(\theta, \mathbf{U}_{0}\right)}{\partial \theta^{2}} \stackrel{\text { def }}{=} D\left(\theta, \mathbf{U}_{0}\right) \\
= & \mathbf{a}^{(2)}(\theta)^{H} \mathbf{U}_{0} \mathbf{W} \mathbf{U}_{0}^{H} \mathbf{a}(\theta)+2 \mathbf{a}^{(1)}(\theta)^{H} \mathbf{U}_{0} \mathbf{W U}_{0}^{H} \mathbf{a}^{(1)}(\theta)+\mathbf{a}(\theta)^{H} \mathbf{U}_{0} \mathbf{W} \mathbf{U}_{0}^{H} \mathbf{a}^{(2)}(\theta)
\end{aligned}
$$

where the superscripts (1) and (2) stand for first- and second-order derivatives, respectively, with respect to the scalar variable $\theta$. Note that $\mathbf{W}$ is not a function of $\theta$, but it could be a function of $U_{o}$, as it is in the case of Min-Norm.

From (4.2)-(4.5), the perturbation in the estimated directions are given as

$$
\Delta \theta_{k}=\frac{N\left(\theta_{k}, \tilde{\mathrm{U}}_{0}\right)}{D\left(\theta_{k}, \tilde{\mathrm{U}}_{0}\right)}, \quad \text { for } \quad k=1, \cdots, P .
$$

Step 2. Linear approximation of the Newton Step with respect to orthogonalsubspace perturbation $\Delta \mathbf{U}_{0}$.

We now approximate the numerator and denominator of $\Delta \theta_{k}$ by first-order perturbation expressions in $\Delta \mathbf{U}_{0}$ in which the nominal term corresponds to the noise-free singular vectors $\mathrm{U}_{o}$,

$$
\begin{aligned}
& N\left(\theta_{k}, \tilde{\mathrm{U}}_{0}\right) \approx N\left(\theta_{k}, \mathrm{U}_{0}\right)+\Delta N \stackrel{\text { def }}{=} N+\Delta N \\
& D\left(\theta_{k}, \tilde{\mathrm{U}}_{0}\right) \approx D\left(\theta_{k}, \mathrm{U}_{0}\right)+\Delta D \stackrel{\text { def }}{=} D+\Delta D .
\end{aligned}
$$


In the above equations, $\Delta N$ and $\Delta D$ are linear functions of $\Delta \mathrm{U}_{0}$. Using the fact that $\mathbf{a}\left(\theta_{k}\right)^{H} \mathbf{U}_{o}=0$, we have that

$$
N\left(\theta_{k}, \mathbf{U}_{0}\right)=0
$$

and

$$
D\left(\theta_{k}, \mathbf{U}_{o}\right)=2 \mathbf{a}^{(1)}\left(\theta_{k}\right)^{H} \mathbf{U}_{o} \mathbf{W} \mathbf{U}_{o}^{H} \mathbf{a}^{(1)}\left(\theta_{k}\right) .
$$

Using (4.6) and (4.7) and the fact the $N=0$, we can write $\Delta \theta_{k}$ as

$$
\begin{aligned}
\Delta \theta_{k} & =\frac{\Delta N}{D+\Delta D} \\
& =\frac{\Delta N}{D(1+\Delta D / D)} \\
& =\frac{\Delta N}{D}\left[1-\frac{\Delta D}{D}+\left(\frac{\Delta D}{D}\right)^{2}-\cdots\right],
\end{aligned}
$$

and keeping only the first-order perturbation yields

$$
\Delta \theta_{k}=\frac{\Delta N}{D}
$$

$D$ has been calculated in (4.8) and it only remains now to calculate $\Delta N$ as a linear function of $\Delta U_{0}$. We first note that $\mathbf{W}$ could be a function of $U_{0}$ (as in MinNorm), and so perturbations in $U_{o}$ induce perturbations in $\mathbf{W}$. We approximate the perturbed $\tilde{\mathbf{w}}$ by a first-order perturbation series as

$$
\tilde{\mathbf{w}} \approx \mathbf{w}+\Delta \mathbf{w}
$$

where $W$ is evaluated at $U_{o}$, and $\Delta W$ is a linear function of $\Delta \mathbf{U}_{o}$. Now we calculate $N\left(\theta_{k}, \tilde{\mathbf{U}}_{o}\right)$ using (4.4)

$$
\begin{aligned}
N\left(\theta_{k}, \mathbf{U}_{0}+\Delta \mathbf{U}_{0}\right)= & -\mathbf{a}^{(1)}\left(\theta_{k}\right)^{H}\left(\mathbf{U}_{0}+\Delta \mathbf{U}_{0}\right)(\mathbf{W}+\Delta \mathbf{W})\left(\mathbf{U}_{0}+\Delta \mathbf{U}_{0}\right)^{H} \mathbf{a}\left(\theta_{k}\right) \\
& -\mathbf{a}\left(\theta_{k}\right)^{H}\left(\mathbf{U}_{0}+\Delta \mathbf{U}_{0}\right)(\mathbf{W}+\Delta \mathbf{W})\left(\mathbf{U}_{0}+\Delta \mathbf{U}_{0}\right)^{H} \mathbf{a}^{(1)}\left(\theta_{k}\right) \\
= & T_{0}+T_{1}+T_{2}+T_{3}
\end{aligned}
$$

where the nominal term $T_{0}=N\left(\theta_{k}, \mathrm{U}_{o}\right)=0, T_{1}$ represents terms in which $\Delta \mathrm{U}_{o}$ appears once, $T_{2}$ represents terms in which $\Delta U_{0}$ appears twice, and $T_{3}$ represents 
terms in which $\Delta \mathbf{U}_{0}$ appears three times. For a first-order perturbation analysis, we ignore the terms in $T_{2}$ and $T_{3}$ and only consider the terms in $T_{1}$. Most of these terms are zero when calculated at $\theta=\theta_{k}$ since they contain the factor $a\left(\theta_{k}\right)^{H} U_{0}$. In particular, all terms in $T_{1}$ containing $\Delta W$ are zero at $\theta=\theta_{k}$. Since the nominal term in (4.11) is zero, terms in $T_{1}$ are the first-order perturbation $\Delta N$ we are looking for. There are only two non-zero terms in $T_{1}$ when $\theta=\theta_{k}$, and the result is

$$
\begin{aligned}
\Delta N & =-\mathbf{a}^{(1)}\left(\theta_{k}\right)^{H} \mathrm{U}_{0} \mathbf{W} \Delta \mathbf{U}_{o}^{H} \mathbf{a}\left(\theta_{k}\right)-\mathbf{a}\left(\theta_{k}\right)^{H} \Delta \mathbf{U}_{0} \mathbf{W} \mathbf{U}_{o}^{H} \mathbf{a}^{(1)}\left(\theta_{k}\right) \\
& =-2 \Re\left[\mathbf{a}\left(\theta_{k}\right)^{H} \Delta \mathbf{U}_{0} \mathbf{W} \mathbf{U}_{o}^{H} \mathbf{a}^{(1)}\left(\theta_{k}\right)\right] .
\end{aligned}
$$

Finally, using (4.8) (4.10) and (4.12) we have the perturbation $\Delta \theta_{k}$ which is linear in the subspace perturbation $\mathrm{U}_{0}$. The result is

$$
\Delta \theta_{k}=\frac{\Re\left[-\mathbf{a}\left(\theta_{k}\right)^{H} \Delta \mathbf{U}_{o} \mathbf{W U}_{o}^{H} \mathrm{a}^{(1)}\left(\theta_{k}\right)\right]}{\mathbf{a}^{(1)}\left(\theta_{k}\right)^{H} \mathbf{U}_{0} \mathbf{W} U_{o}^{H} \mathbf{a}^{(1)}\left(\theta_{k}\right)} .
$$

We now substitute (3.15) into the above equation to get an expression

$$
\Delta \theta_{k}=\frac{\Re\left[a\left(\theta_{k}\right)^{H} U_{,} \Sigma_{o}^{-1} \mathbf{V}_{e}^{H} N_{0} \mathbf{W U}_{o}^{H} \mathbf{a}^{(1)}\left(\theta_{k}\right)\right]}{\mathbf{a}^{(1)}\left(\theta_{k}\right)^{H} \mathrm{U}_{0} \mathbf{W U}_{o}^{H} \mathbf{a}^{(1)}\left(\theta_{k}\right)} .
$$

To simplify notation, we define the vectors

$$
\begin{aligned}
& \alpha_{k}=\mathrm{V}_{0} \mathbf{\Sigma}^{-1} \mathrm{U}_{a}^{H} \mathrm{a}\left(\theta_{k}\right) \\
& \beta_{k}=\mathrm{U}_{0} \mathbf{W} \mathbf{U}_{0}^{H} \mathrm{a}^{(1)}\left(\theta_{k}\right)
\end{aligned}
$$

and the scalar

$$
\gamma_{k}=\mathbf{a}^{(1)}\left(\theta_{k}\right)^{H} U_{0} \mathbf{W} U_{0}^{H} a^{(1)}\left(\theta_{k}\right)
$$

Then we have

$$
\Delta \theta_{k}=\frac{\Re\left[\alpha_{k} N \beta_{k}\right]}{\gamma_{k}}
$$




\subsection{Statistical Performance}

Since we have used a first-order perturbation analysis, the predicted bias of an estimated direction of arrival is

$$
E\left(\Delta \theta_{k}\right)=\frac{\Re\left[\alpha_{k} E(N) \beta_{k}\right]}{\gamma_{k}}=0 .
$$

The mean-squared error of an estimated direction of arrival by

$$
E\left(\Delta \theta_{k}\right)^{2}=\frac{E\left[\Re\left(\alpha_{k}^{H} \mathrm{~N} \beta_{k}\right)\right]^{2}}{\gamma_{k}^{2}} .
$$

It is shown in Appendix $A$ that if the real and imaginary parts of the noise matrix $\mathrm{N}$ are independent Gaussian random variables with zero mean and variance $\sigma_{n}^{2} / 2$, then

$$
E\left[\Re\left(\alpha_{k}^{H} \mathrm{~N} \beta_{k}\right)\right]^{2}=\frac{1}{2}\left\|\alpha_{k}\right\|^{2}\left\|\beta_{k}\right\|^{2} \sigma_{n}^{2} .
$$

Thus we have the result that

$$
E\left(\Delta \theta_{k}\right)^{2}=\frac{\left\|\alpha_{k}\right\|^{2}\left\|\beta_{k}\right\|^{2} \sigma_{n}^{2}}{2 \gamma_{k}^{2}} .
$$

MUSIC (SMS): the weighting matrix $\mathbf{W}=\mathbf{I}$, so that

$$
\left\|\beta_{k}\right\|^{2}=\mathbf{a}^{(1)}\left(\theta_{k}\right)^{H} \mathbf{U}_{0} \mathbf{U}_{o}^{H} a^{(1)}\left(\theta_{k}\right) .
$$

Then the mean-squared error expression becomes

$$
\begin{aligned}
E\left(\Delta \theta_{k}\right)_{S M S}^{2} & =\frac{\left\|\alpha_{k}\right\|^{2} \sigma_{n}^{2}}{2\left[\mathbf{a}^{(1)}\left(\theta_{k}\right)^{H} \mathrm{U}_{0} \mathbf{U}_{o}^{H} \mathbf{a}^{(1)}\left(\theta_{k}\right)\right]} \\
& =\frac{\left\|\alpha_{k}\right\|^{2} \sigma_{n}^{2}}{2\left\|\mathbf{a}^{(1)}\left(\theta_{k}\right)^{H} \mathbf{U}_{o}\right\|^{2}} .
\end{aligned}
$$

Min-Norm (SMN): the weighting matrix $\mathbf{W}=\boldsymbol{c} \boldsymbol{c}^{H}$, so that

$$
\left\|\beta_{k}\right\|^{2}=\bar{c}^{H} \bar{c}\left[\mathrm{a}^{(1)}\left(\theta_{k}\right)^{H} \mathbf{U}_{0} \overline{c c}^{H} \mathbf{U}_{o}^{H} \mathbf{a}^{(1)}\left(\theta_{k}\right)\right] .
$$

Then the mean-squared error expression becomes

$$
\begin{aligned}
E\left(\Delta \theta_{k}\right)_{S M N}^{2} & =\frac{\left\|\alpha_{k}\right\|^{2}\|\mathbf{c}\|^{2} \sigma_{n}^{2}}{2\left[\mathbf{a}^{(1)}\left(\theta_{k}\right)^{H} U_{o} \overline{c k}^{H} U_{o}^{H} \mathbf{a}^{(1)}\left(\theta_{k}\right)\right]} \\
& =\frac{\left\|\alpha_{k}\right\|^{2}\|\mathbf{c}\|^{2} \sigma_{n}^{2}}{2\left|\mathbf{a}^{(1)}\left(\theta_{k}\right)^{H} U_{o} \bar{c}\right|^{2}}
\end{aligned}
$$


or equivalently

$$
E\left(\Delta \theta_{k}\right)_{S M N}^{2}=\frac{\left\|\alpha_{k}\right\|^{2}\|\mathrm{~d}\|^{2} \sigma_{n}^{2}}{2\left|\mathbf{a}^{(1)}\left(\theta_{k}\right)^{H} U_{0} \mathbf{c}\right|^{2}}
$$

In above analysis, only the first-order partial derivative of $a(\theta)$ with respect to $\theta$ is needed. When this derivative is evaluated at a true direction $\theta_{k}$, the result is

$$
a_{i}^{(1)}\left(\theta_{k}\right)=e^{j \frac{2 \pi}{\lambda}\left(x_{i} \sin \theta_{k}+y_{i} \cos \theta_{k}\right)} j \frac{2 \pi}{\lambda}\left(x_{i} \cos \theta_{k}-y_{i} \sin \theta_{k}\right) .
$$

In the special case of a uniform line array, $a_{i}\left(\theta_{k}\right)$ and $a_{i}^{(1)}\left(\theta_{k}\right)$ reduce to

$$
\begin{gathered}
a_{i}\left(\theta_{k}\right)=e^{j \frac{j z}{\lambda z}(i-1) \sin \theta_{k}} \\
a_{i}^{(1)}\left(\theta_{k}\right)=e^{j \frac{25}{\lambda z}(i-1) \sin \theta_{k}} j \frac{2 \pi d}{\lambda}(i-1) \cos \theta_{k} .
\end{gathered}
$$

where $d$ is the spacing between adjacent sensors.

\subsection{Analytical Comparison of MUSIC and Min- Norm}

If we divide (4.19) by (4.18) and use the Schwarz inequality

$$
\left|\mathbf{x}^{H} \mathbf{y}\right|^{2} \leq\|\mathbf{x}\|^{2}\|\mathbf{y}\|^{2}
$$

it can be seen that

$$
\begin{aligned}
\frac{E\left(\Delta \theta_{k}\right)_{S M N}^{2}}{E\left(\Delta \theta_{k}\right)_{S M S}^{2}} & =\frac{\left\|\mathbf{a}^{(1)}\left(\theta_{k}\right)^{H} \mathrm{U}_{0}\right\|^{2}\|\mathbf{c}\|^{2}}{\left|\mathbf{a}^{(1)}\left(\theta_{k}\right)^{H} \mathrm{U}_{0} \bar{c}\right|^{2}} \\
& \geq \frac{\left\|\mathbf{a}^{(1)}\left(\theta_{k}\right)^{H} U_{o}\right\|^{2}\|\bar{c}\|^{2}}{\left\|\mathbf{a}^{(1)}\left(\theta_{k}\right)^{H} U_{0}\right\|^{2}\|\mathbf{c}\|^{2}} \\
& =1 .
\end{aligned}
$$

In general, the mean-squared error of the estimated directions-of-arrivals from the Min-Norm algorithm are lower-bounded by the corresponding mean-squared error from MUSIC. In the above equations we are making the correspondence

$$
\mathbf{x}^{H}=\mathbf{a}^{(1)}\left(\theta_{k}\right)^{H} \mathrm{U}_{0}
$$




\begin{tabular}{|c|c|c|}
\hline & SMS & SMN \\
\hline$\left\|\alpha_{k}\right\|^{2}$ & $\left\|\mathbf{a}\left(\theta_{k}\right)^{H} \mathbf{U}_{s} \Sigma_{j}^{-1} \mathbf{V}_{s}\right\|^{2}$ & $\left\|\mathbf{a}\left(\theta_{k}\right)^{H} \mathbf{U}_{s} \Sigma_{s}^{-1} \mathbf{V}_{s}\right\|^{2}$ \\
\hline$\left\|\beta_{k}\right\|^{2}$ & $\left\|\mathbf{a}^{(1)}\left(\theta_{k}\right)^{H} \mathbf{U}_{o}\right\|^{2}$ & $\left\|\mathbf{a}^{(1)}\left(\theta_{k}\right)^{H} \mathbf{U}_{o}\right\|^{2}\|\mathbf{c}\|^{2}$ \\
\hline$\gamma_{k}$ & $\left\|\mathbf{a}^{(1)}\left(\theta_{k}\right)^{H} \mathbf{U}_{o}\right\|^{2}$ & $\left\|\mathbf{a}^{(1)}\left(\theta_{k}\right)^{H} \mathbf{U}_{o} \mathbf{c}\right\|^{2}$ \\
\hline
\end{tabular}

Table 4.1: Parameters of mean-squared error for Extrema Search Algorithms

$$
\mathbf{y}=\mathbf{c} .
$$

The condition for equality in (4.22) is that the vector $y$ be proportional to $x$. Since $\mathrm{c}^{H}$ is proportional to the first row of $\mathrm{U}_{0}$, the condition for equality in equation (4.23) is that

$$
\mathrm{a}^{(1)}\left(\theta_{k}\right)=\left(\begin{array}{llll}
\delta & 0 & \ldots & 0
\end{array}\right)^{T},
$$

where $\delta$ is any non-zero constant. However, by examining (4.21), it can be seen that the zero elements in (4.24) cannot be achieved for arbitrary source locations.

\subsection{Numerical Examples}

In this section, we give a simulation example to demonstrate the algorithms as well as to verify the performance analysis. A twenty-element uniform circular array (shown in Figure 4.1) is used where the distance between adjacent sensors is one half the signal wavelength. The first sensor of the array is located at the origin of the $X-Y$ plane, and angles are measured with respect to the $Y$ axis. Two sources are considered at 0.2 and 0.5 radians. Twenty snapshots of array data were used to estimate the directions of arrival for a given trial, data matrices of dimension $20 \times 20$ were formed, and 100 trials were run at each value of SNR. Here SNR is defined as $10 \log \left(\frac{1}{\sigma_{n}^{2}}\right)$ where the $\sigma_{n}^{2}$ is the variance of the complex, additive noise. The data is generated according to the following formula

$$
y_{i}(n)=\sum_{k=1}^{2} e^{j \frac{2 \pi}{\lambda_{0}}\left(z_{i} \sin \theta_{k}+y_{i} \cos \theta_{k}\right)+\phi_{k n}}+n_{i}(n)
$$


where $\left(x_{i}, y_{i}\right)$ are the sensor locations, $\lambda_{c}$ is the center wavelength of the narrowband signal, and $\phi_{k n}$ are independent random phase angles uniformly distributed in the interval $(-\pi, \pi)$. Figure 4.2 shows a null-spectrum function used in the extrema search approach of subspace based algorithms.

The theoretical mean-squared error for each estimated direction was computed using (4.18) and (4.19) for each trial. The theoretical mean-squared error is a random variable because the signal is random. The mean and standard deviation of the theoretical mean-squared error were computed for each set of 500 trials, and it was found that the standard deviation was less the $5 \%$ of the mean. Thus in the figures, we only plot the mean of theoretical mean-squared error.

Figures 4.3 and 4.4 show the root-mean-squared error of the estimated directions versus SNR. The simulation results (shown with an $\star$ ) agree closely with theoretical predictions (shown by the solid line).

\subsection{Summary}

In this chapter, a non-asymptotic statistical performance analysis using matrix approximation on a common model for orthogonal-subspace based extrema searching methods (MUSIC and Min-Norm searching algorithm) with arbitrary array geometry has been developed analytically and verified by simulations. The analysis used one Newton-Raphson step and a first-order Taylor expansion. The formula for the perturbation of the orthogonal-subspace derived in chapter 3 was utilized to unify the analyses. The tractable formulas for the mean-squared error of DOA estimates of various orthogonal-subspace based methods were derived. These formulas show that the mean-squared error of estimated directions of arrival from the Min-Norm method is not smaller than that from MUSIC. 


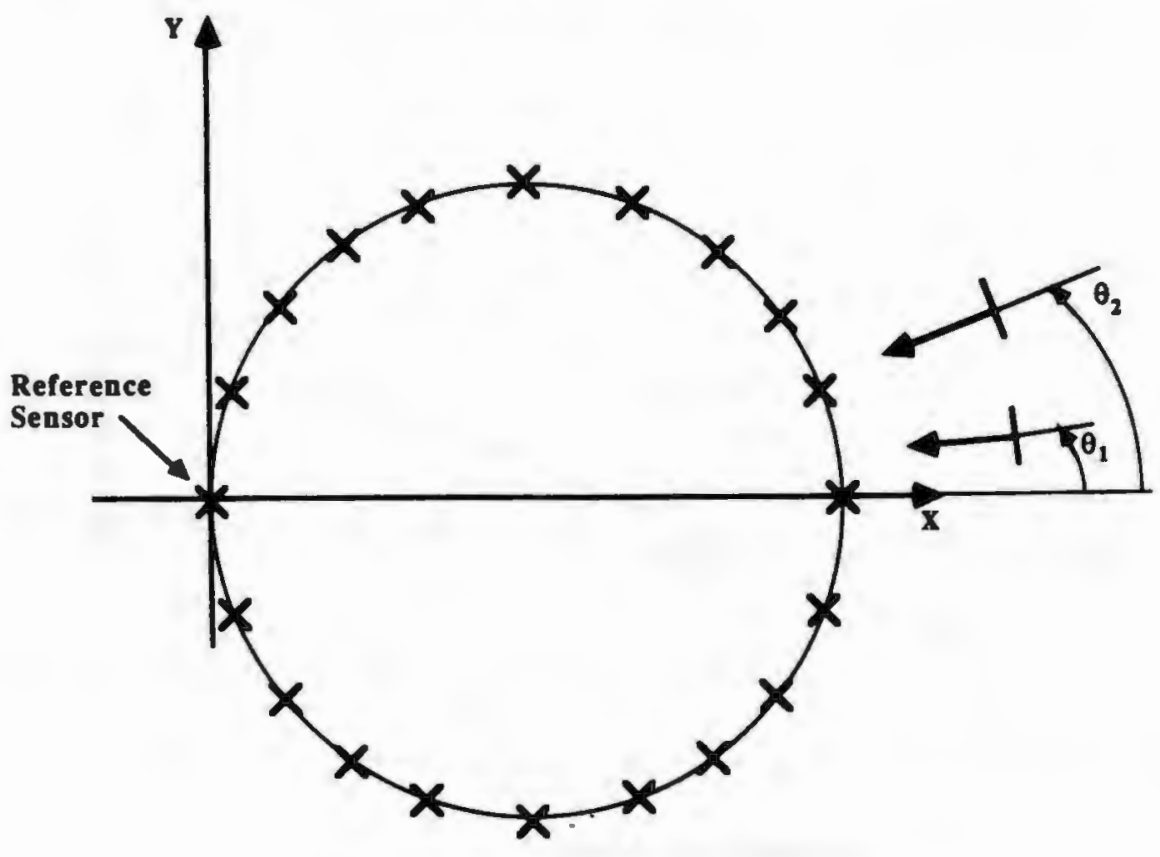

Figure 4.1: Uniform circular array

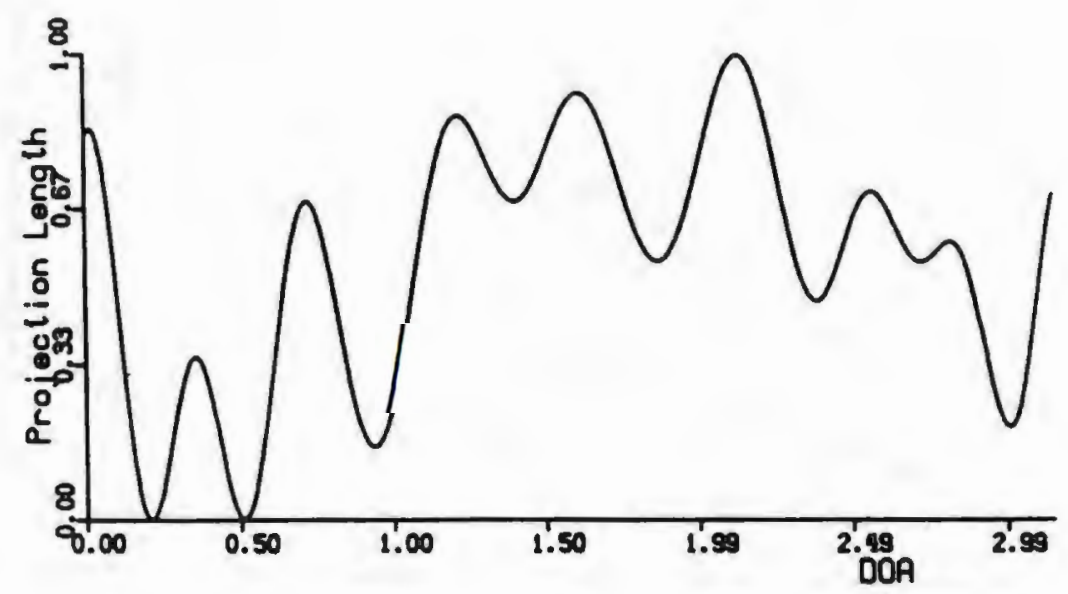

Figure 4.2: Null-spectrum function of Extrema Search Approach 


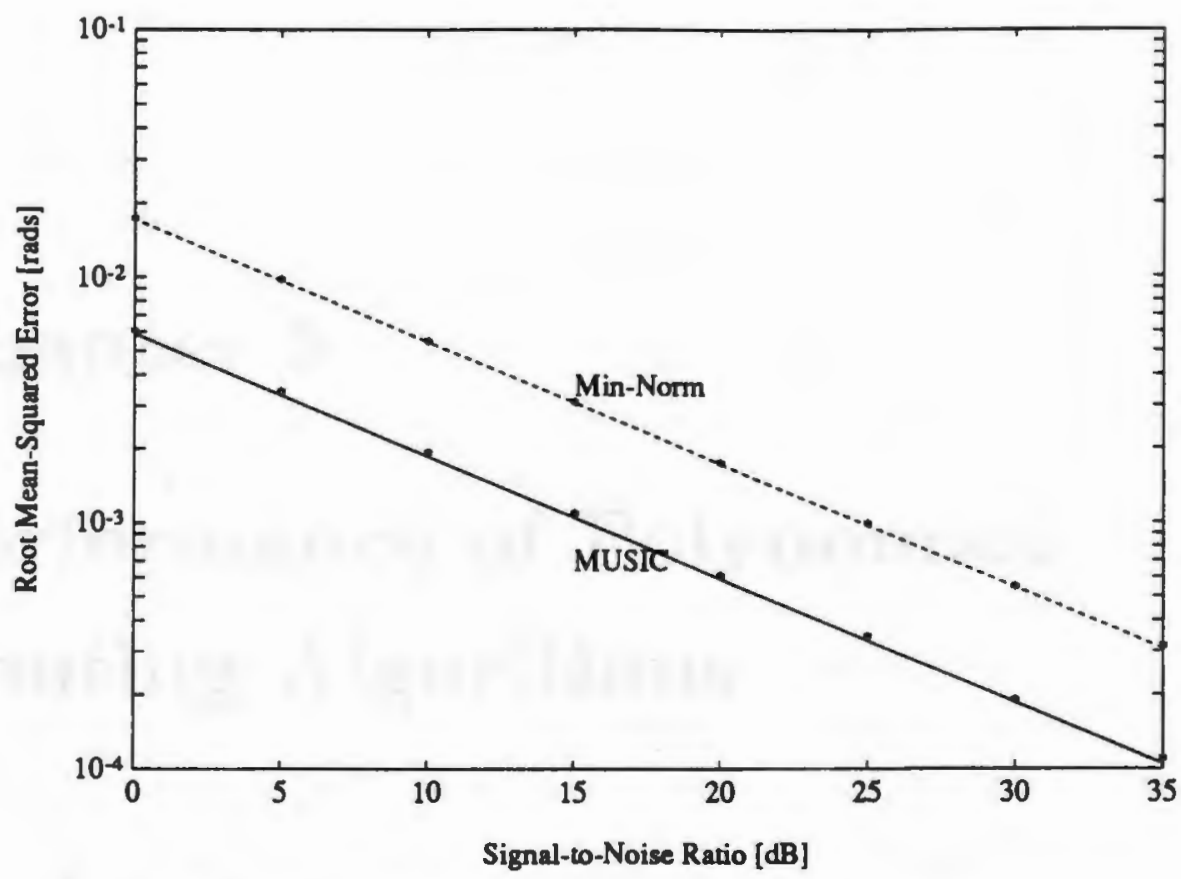

Figure 4.3: RMSE vs. SNR for MUSIC and Min-Norm at source 1 (.2 rads)

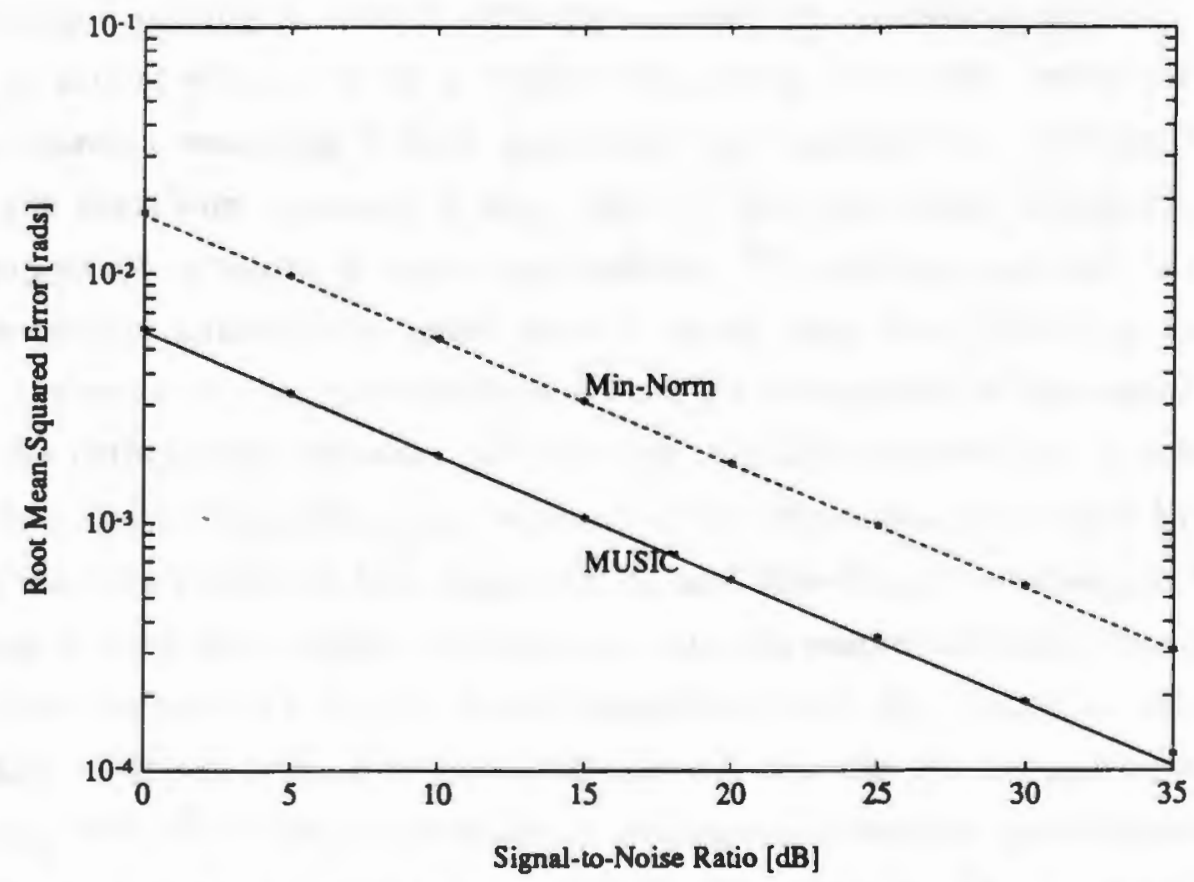

Figure 4.4: RMSE vs. SNR for MUSIC and Min-Norm at source 2 (.5 rads) 


\section{Chapter 5}

\section{Performance of Polynomial}

\section{Rooting Algorithms}

\subsection{Introduction}

Polynomial rooting is another orthogonal-subspace processing approach to direction of arrival estimation for a uniform line array, which has better performance than extrema searching if both approaches are applied to a uniform line array. But the searching approach is also valid for arbitrary array geometry, which is an important property in many applications. The rooting approach is similar to the searching approach in many aspects except that the DOA's are determined from the roots of a polynomial formed from the intersection of the signal manifold and the orthogonal-subspace. MUSIC was originally proposed as a searching algorithm; the uniform line array version - Root-Music was developed by Barabell [1]. Pisarenko's Method (see Appendix A) and Min-Norm were proposed as polynomial rooting approaches, and later an extrema search approach was developed for these methods $[17,60,8]$. Search algorithms look for minima or maxima of a function in one-dimension so that finite sample bias can be induced; however, this is easily seen not to be a limitation of orthogonal-subspace approach per se, but rather a consequence of using a computationally attractive one-dimensional search. 
The rooting algorithms are better suited to the geometric nature of the problem in the sense that they actually look for a global minimum or maximum over a multi-dimension space. In other words, all the roots of the polynomial are simultaneously sought. This is important because distinct roots do not imply distinct extrema in the spectrum function. If there are two closely spaced roots, the spectrum may have only one extremum resulting in an apparent loss in resolution using a search procedure. However, the rooting approach can obtain (and resolve) true roots. Therefore, locating the roots, and using their angular location to obtain the DOA's is preferable in the case where the estimation is performed near threshold. In general, for a polynomial with the order greater than 3 , iterative root finding techniques are required, which means the improvement of resolution is gained at a high expense of costly computation. Besides, as long as the roots are distinct, i.e., above threshold, the extrema-search algorithm has the same performance as polynomial-rooting algorithm, as we shall show in this chapter.

There have been many analyses of the polynomial-rooting approach of MinNorm method $[2,62,6,16,60,8,53,5]$, while few analyses on Root-MUSIC [50]. We have developed a unified analysis of the polynomial-rooting approach [63] which we will elaborate on in this chapter.

In this chapter, we will first develop a common form of these algorithms for analysis (Section 5.2); then we provide a first-order relation between subspace perturbations and signal-root perturbations (Section 5.3). This is done with some care because the signal roots of the spectral polynomial always have multiplicity two. The statistical performance of the algorithms and the relation to the extremasearch approach, will be derived in section 5.4 and 5.5, respectively. Finally, in Section 5.6, the perturbation of signal roots of the spectral polynomial wil be discussed.

\subsection{Common Model}

Min-Norm and Root-MUSIC methods estimate the directions of arrival using polynomial rooting when the sensor array has a uniform line geometry. Under the 
constraint of uniform line structure, the array manifold is

$$
a\left(z^{-1}\right)^{T}=\left(\begin{array}{llll}
1 & z^{-1} & \ldots & z^{-L+1}
\end{array}\right)
$$

where $z=e^{j \frac{2 \pi d}{\lambda_{e}} \sin \theta}$ and $d$ is the spacing between adjacent sensors. The product of the array manifold and orthogonal-subspace vectors $a\left(z^{-1}\right)^{T} U_{0}$ can be viewed as a Z-transform of the columns of $\mathbf{U}_{0}$. Using the orthogonality relationship (3.3), these Z-transforms equal zero at $z_{k}=e^{j \frac{3 x_{e}}{\lambda_{e}} \sin \theta_{k}}$,

$$
\mathbf{a}\left(z_{k}\right)^{H} \mathrm{U}_{o}=0
$$

where the $\theta_{k}$ 's are directions of arrivals.

A common model for MUSIC and Min-Norm (also Pisarenko's method) can be written in terms of spectral polynomial

$$
\begin{aligned}
P(z) & =\mathrm{a}\left(z^{-1}\right)^{T} \mathrm{U}_{0} \mathrm{WU}_{o}^{H} \mathrm{a}(z) \\
& =A \prod_{i=1}^{L-1}\left(1-r_{i} z^{-1}\right)\left(1-r_{i}^{*} z\right)
\end{aligned}
$$

where $\mathbf{W}$ is a weighting matrix and $A$ is a scalar factor. For future reference we define the signal roots of $P(z)$ to be $\left\{r_{i}\right\}, i=1, \cdots, P$. These roots correspond to the $P$ different sources, and are on the unit circle. The other roots $\left\{r_{i}\right\}, i=$ $P+1, \cdots, L$ are not on the unit circle. Min-Norm and Root-MUSIC can be obtained as special cases of (5.1) and (5.2) by choosing $W$ and $A$ as follows.

Min-Norm

$$
\mathbf{W}=\boldsymbol{c c}^{B} \text { and } A=1 .
$$

Note that the choice of $\mathbf{W}$ and $A$ yields the spectral polynomial $P(z)$ corresponding to Min-Norm. Half of the roots of $P(z)$ are identical to the roots of Min-Norm polynomial in (2.25), and the other half are at locations reflected through the unit circle.

$\underline{\text { Root-MUSIC }}$

$$
\mathbf{W}=\mathbf{I} \text { and } A=\frac{(L-P)}{\|\mathbf{h}\|^{2}}
$$


where $\mathbf{b}$ is the coefficient vector (with first element of unity) for the polynomial $H(z) . H(z)$ is the causal spectral factor of $P(z)$, namely, $P(z)=H(z) H\left(z^{-1}\right)^{*}$. The vector $h$ can be easily obtained from the roots $\left\{r_{i}\right\}$.

\subsection{Perturbation of the Angle Estimates}

In Chapter 3, a formula was given which showed, to first-order, the perturbation in the orthogonal subspace. Here we show how the subspace perturbation induces perturbations in the estimated directions of arrival. The noise-free spectral polynomial $P(z)$ has roots at $z_{k}=e^{j \frac{2 \pi t}{\lambda_{e}} \sin \theta_{k}}$ where $\theta_{k}$ are the DOA's. The root locations of $P(z)$ are local minima since $P(z)$ is nonnegative. When the spectral polynomial is perturbed, the locations of local minima change slightly, and in general, the new local minima are not equal to zero (i.e. the perturbed spectral polynomial does not have roots on the unit circle).

The locations of the local minima of either the noise-free or perturbed spectral polynomials are obtained by setting the first derivative equal to zero. In other words, the roots of the derivative of $P(z)$ give the locations of the relative minima (and maxima) of $P(z)$. Thus to find the perturbations of the DOA's (which correspond to minima of $P(z)$ ), we must calculate the perturbations of the roots of the derivative of $P(z)$. We are only interested in the roots at $z_{k}=e^{j \frac{2 \pi d}{\lambda_{c}} \sin \theta_{k}}$ corresponding to noise-free DOA's.

These roots will be on the unit-circle if the null-spectrum has distinct minima, and off the unit circle otherwise. Since it is only the angle of the roots which contains DOA information, it does not matter if the roots are on the unit circle or not, and so the analysis given in the this section involving the derivative of the spectral polynomial is appropriate whether or not the null spectrum has distinct minima.

The discussion above considers the case when the perturbed null-spectrum has distinct minima for each DOA. However, we However, we have pointed out previous that this is not necessary for polynomial rooting to work. In the case when the perturbed null-spectrum polynomial does not possess distinct minima for each 
DOA, it is still appropriate to look at the roots of the derivative of the spectral polynomial.

In what follows, we calculate the perturbations in the roots of the derivative of the spectral polynomial as a function of the perturbation in the orthogonal subspace. The following steps are used in the calculation:

1. Derive a first-order expression of $\frac{\partial P(z)}{\partial z}$ as a function of the perturbation of the orthogonal subspace.

2. Derive a first-order expression for the perturbation of $\frac{\partial P(z)}{\partial z}$ as a function of the perturbation in the signal-roots.

3. Equate the expressions derived in 1 and 2 above to get the desired relationship between subspace and signal-root perturbations.

Step 1. Perturbation of the derivative of the spectral polynomial as a function of the orthogonal-subspace perturbations.

We start with

$$
P\left(z, \tilde{\mathrm{U}}_{0}\right)=\mathbf{a}\left(z^{-1}\right)^{T} \tilde{\mathbf{U}}_{0} \tilde{\mathbf{W}}_{0}^{H} \mathbf{a}(z) .
$$

The first derivative with respect to $z$ is

$$
\frac{\partial P\left(z, \tilde{\mathbf{U}}_{o}\right)}{\partial z}=-z^{-2} \mathrm{a}^{(1)}\left(z^{-1}\right)^{T} \tilde{\mathrm{U}}_{0} \tilde{\mathbf{W}} \tilde{\mathrm{U}}_{o}^{H} \mathrm{a}(z)+\mathbf{a}\left(z^{-1}\right)^{T} \tilde{\mathrm{U}}_{0} \tilde{\mathbf{W}} \tilde{\mathrm{U}}_{o}^{H} \mathrm{a}^{(1)}(z)
$$

where

$$
\mathrm{a}^{(1)}(z)=\frac{d \mathrm{a}(z)}{d z}=\left(\begin{array}{lllll}
0 & 1 & 2 z & \cdots & (L-1) z^{L-2}
\end{array}\right)^{T} .
$$

Substitute $\tilde{\mathbf{U}}_{o}=\mathbf{U}_{o}+\Delta \mathbf{U}_{0}$ and $\tilde{\mathbf{W}}=\mathbf{W}+\Delta \mathbf{W}$ into (5.4) to obtain

$$
\begin{aligned}
\frac{\partial P\left(z, \tilde{U}_{0}\right)}{\partial z}= & -z^{-2} \mathbf{a}^{(1)}\left(z^{-1}\right)^{T}\left(\mathbf{U}_{0}+\Delta \mathbf{U}_{0}\right)(\mathbf{W}+\Delta \mathbf{W})\left(\mathbf{U}_{0}+\Delta \mathbf{U}_{0}\right)^{H} \mathbf{a}(z) \\
& +\mathbf{a}\left(z^{-1}\right)^{T}\left(\mathbf{U}_{0}+\Delta \mathbf{U}_{0}\right)(\mathbf{W}+\Delta \mathbf{W})\left(\mathrm{U}_{0}+\Delta \mathbf{U}_{0}\right)^{H} \mathbf{a}^{(1)}(z) \\
= & T_{0}+T_{1}+T_{2}+T_{3}
\end{aligned}
$$

where $T_{0}$ represents terms in which $\Delta \mathrm{U}_{0}$ does not appear, $T_{1}$ represents terms in which $\Delta \mathbf{U}_{0}$ appears once, $T_{2}$ represents terms in which $\Delta \mathbf{U}_{0}$ appears twice, and $T_{3}$ 
represents terms in which $\Delta \mathbf{U}_{0}$ appears three times. For a first-order perturbation analysis, we ignore the terms in $T_{2}$ and $T_{3} . T_{0}$ is the nominal term which equals zero, and so we only need to consider the terms in $T_{1}$. Most of these terms are zero when calculated at $z=r_{k}$ since they contain the factor $\mathrm{a}\left(z^{-1}\right)^{T} \mathrm{U}_{0}$. In particular, all terms in $T_{1}$ containing $\Delta W$ are zero at $z=r_{k}$. There are only two non-zero terms in $T_{1}$ when $z=r_{k}$, and the result is that to the first order in $\Delta \mathrm{U}_{o}$

$$
\begin{aligned}
& \left.\frac{\partial P\left(z, \tilde{U}_{o}\right)}{\partial z}\right|_{z=r_{k}} \\
& =\left.\left[-z^{-2} \mathbf{a}^{(1)}\left(z^{-1}\right)^{T} \mathbf{U}_{0} \mathbf{W} \Delta \mathbf{U}_{0}^{H} \mathbf{a}(z)+\mathbf{a}\left(z^{-1}\right)^{T} \Delta \mathbf{U}_{0} \mathbf{W} \mathbf{U}_{0}^{H} \mathbf{a}^{(1)}(z)\right]\right|_{z=r_{k}} \\
& =-r_{k}^{*}\left[r_{k}^{*} \mathrm{a}^{(1)}\left(r_{k}^{*}\right)^{T} \mathrm{U}_{0} \mathrm{~W} \Delta \mathrm{U}_{0}^{H} \mathrm{a}\left(r_{k}\right)-r_{k} \mathrm{a}\left(r_{k}^{*}\right)^{T} \Delta \mathrm{U}_{0} \mathrm{WU}_{o}^{H} \mathrm{a}^{(1)}\left(r_{k}\right)\right] \\
& =-2 j r_{k}^{*} \Im\left[r_{k}^{*} \mathrm{a}\left(r_{k}^{-1}\right)^{T} \Delta \mathrm{U}_{0} \mathrm{WU}_{o}^{H} \mathrm{a}^{(1)}\left(r_{k}\right)\right] \text {. }
\end{aligned}
$$

Step 2. Perturbation of the derivative of the spectral polynomial as a function of root perturbations.

We now investigate how the perturbation of the orthogonal subspace induces a perturbation in $\frac{\partial P(z)}{\partial z}$. We write the perturbed polynomial as

$$
P(z, \tilde{r})=A \prod_{i=1}^{L-1}\left(1-\tilde{r}_{i} z^{-1}\right)\left(1-\tilde{r}_{i}^{*} z\right)
$$

and take the derivative of $P(z, \tilde{r})$ over $z$ to get

$$
\begin{aligned}
\frac{\partial P(z, \tilde{r})}{\partial z} & =A \sum_{i=1}^{L-1}\left[\tilde{r}_{i} z^{-2}\left(1-\tilde{r}_{i}^{*} z\right)-\tilde{r}_{i}^{*}\left(1-\tilde{r}_{i} z^{-1}\right)\right] G\left(z, \tilde{r}_{i}\right) \\
& =A z^{-1} \sum_{i=1}^{L-1}\left[\tilde{r}_{i} z^{-1}\left(1-\tilde{r}_{i}^{*} z\right)-\tilde{r}_{i}^{*} z\left(1-\tilde{r}_{i} z^{-1}\right)\right] G\left(z, \tilde{r}_{i}\right)
\end{aligned}
$$

where

$$
G\left(z, \tilde{r}_{i}\right) \stackrel{\text { def }}{=} \prod_{\substack{j \neq i \\ j=1}}^{L-1}\left(1-\tilde{r}_{j} z^{-1}\right)\left(1-\tilde{r}_{j}^{*} z\right) .
$$

Substitute $\tilde{r}=r+\Delta r$ into above equation

$$
\frac{\partial P(z, \tilde{r})}{\partial z}=A z^{-1} \sum_{i=1}^{L-1}\left\{\left(r_{i}+\Delta r_{i}\right) z^{-1}\left[1-\left(r_{i}+\Delta r_{i}\right)^{*} z\right]\right.
$$




$$
\begin{aligned}
& \left.-\left(r_{i}+\Delta r_{i}\right)^{*} z\left[1-\left(r_{i}+\Delta r_{i}\right) z^{-1}\right]\right\} G\left(z, \tilde{r}_{i}\right) \\
& =A z^{-1} \sum_{i=1}^{L-1} S\left(z, \tilde{r}_{i}\right) G\left(z, \tilde{r}_{i}\right)
\end{aligned}
$$

where

$$
S\left(z, \tilde{r}_{i}\right)=\tilde{r}_{i} z^{-1}\left[1-\tilde{r}_{i}^{*} z\right]-\tilde{r}_{i}^{*} z\left[1-\tilde{r}_{i} z^{-1}\right] .
$$

We want to obtain an expression for (5.10) which contains only first-order terms in $\Delta r_{i}$. We first expand $G\left(z, \tilde{r}_{i}\right)$ and $S\left(z, \tilde{r}_{i}\right)$ in perturbation series in $\Delta r_{i}$ as follows

$$
\begin{aligned}
& G\left(z, \tilde{r}_{i}\right)=G\left(z, r_{i}\right)+\Delta G\left(z, r_{i}\right)+o\left(\Delta r_{i}^{2}\right) \\
& S\left(z, \tilde{r}_{i}\right)=S\left(z, r_{i}\right)+\Delta S\left(z, r_{i}\right)+o\left(\Delta r_{i}^{2}\right)
\end{aligned}
$$

where $\Delta G\left(z, r_{i}\right)$ and $\Delta S\left(z, r_{i}\right)$ are linear functions of $\Delta r_{i}$. Substituting (5.12) into (5.10) yields 


$$
\begin{aligned}
& \frac{\partial P(z, \tilde{r})}{\partial z} \\
= & A z^{-1} \sum_{i=1}^{L-1}\left[S\left(z, r_{i}\right)+\Delta S\left(z, r_{i}\right)+o\left(\Delta r_{i}^{2}\right)\right]\left[G\left(z, r_{i}\right)+\Delta G\left(z, r_{i}\right)+o\left(\Delta r_{i}^{2}\right)\right] \\
= & A z^{-1} \sum_{i=1}^{L-1}\left[S\left(z, r_{i}\right) G\left(z, r_{i}\right)+\Delta S\left(z, r_{i}\right) G\left(z, r_{i}\right)+S\left(z, r_{i}\right) \Delta G\left(z, r_{i}\right)+o\left(\Delta r_{i}^{2}\right)\right] .
\end{aligned}
$$

In order to evaluate (5.13), we need expressions for $\Delta G\left(z, r_{i}\right)$ and $\Delta S\left(z, r_{i}\right)$. From (5.11)

$$
\begin{aligned}
S\left(z, \tilde{r}_{i}\right)= & \left(r_{i}+\Delta r_{i}\right) z^{-1}\left[1-\left(r_{i}+\Delta r_{i}\right)^{*} z\right]-\left(r_{i}+\Delta r_{i}\right)^{*} z\left[1-\left(r_{i}+\Delta r_{i}\right) z^{-1}\right] \\
= & \left.S\left(z, r_{i}\right)+\Delta r_{i} z^{-1}\left(1-r_{i}^{*} z\right)-r_{i} z^{-1} \Delta r_{i}^{*} z\right]-\Delta r_{i}^{*} z^{-1}\left(1-r_{i} z^{-1}\right) \\
& +r_{i}^{*} z \Delta r_{i} z^{-1}+o\left(\Delta r_{i}^{2}\right) .
\end{aligned}
$$

Collecting the terms in the above equation which are linear in $\Delta r_{i}$ and simplifying gives the following expression for $\Delta S\left(z, r_{i}\right)$ :

$$
\Delta S\left(z, r_{i}\right)=\Delta r_{i} z^{-1}+\Delta r_{i}^{*} z .
$$

A similar calculation for $\Delta G\left(z, r_{i}\right)$ yields

$$
\Delta G\left(z, r_{i}\right)=\prod_{\substack{j \neq i \\ j=1}}^{L-1}\left(1-r_{j} z^{-1}\right) \Delta r_{i}^{*} z-\Delta r_{i} z^{-1}\left(1-r_{j}^{*} z\right) .
$$

If we now evaluate (5.9) (5.11) and (5.12) at the signal roots of $P(z)$, namely $r_{k}, k=1, \cdots, P$ and note that $z^{-1}=r_{k}^{*}$ (since $r_{k}$ is on unit circle), then it can be shown that

$$
\begin{aligned}
& \left.S\left(z, r_{i}\right)\right|_{z=r_{k}}=0, \quad i=k \\
& \left.G\left(z, r_{i}\right)\right|_{z=r_{k}}=0, \quad i \neq k \\
& \left.\Delta G\left(z, r_{i}\right)\right|_{z=r_{k}}=0, \quad i \neq k .
\end{aligned}
$$


Substituting the above formulas into (5.13) and keeping only the first-order terms yields

$$
\begin{aligned}
\left.\frac{\partial P(z, \tilde{r})}{\partial z}\right|_{z=r_{k}} & =A r_{k}^{*}\left[\Delta S\left(z, r_{k}\right) G\left(z, r_{k}\right)\right] \\
& =A r_{k}^{*}\left[\Delta r_{k} r_{k}^{*}-\Delta r_{k}^{*} r_{k}\right] G\left(r_{k}, r_{k}\right) \\
& =2 j A r_{k}^{*} \Im\left(\Delta r_{k} r_{k}^{*}\right) G\left(r_{k}, r_{k}\right) .
\end{aligned}
$$

Step 3. Equating the perturbation of the derivative of the spectral polynomial as a function of signal-roots and orthogonal-subspace perturbations.

From (5.18) and (5.7), we obtain

$$
\begin{gathered}
2 j A r_{k}^{*} \Im\left(\frac{\Delta r_{k}}{r_{k}}\right) G\left(r_{k}\right) \\
=-2 j r_{k}^{*} \Im\left[r_{k}^{*} a\left(r_{k}^{-1}\right)^{T} \Delta U_{0} W U_{0}^{H} a^{(1)}\left(r_{k}\right)\right]
\end{gathered}
$$

or

$$
\Im\left(\frac{\Delta r_{k}}{r_{k}}\right)=-\frac{\Im\left[\mathrm{a}\left(r_{k}^{-1}\right)^{T} \Delta \mathrm{U}_{0} \mathrm{WU}_{0}^{H} \mathrm{a}^{(1)}\left(r_{k}\right)\right]}{A r_{k} G\left(r_{k}\right)}
$$

where we use $r_{k}^{*}=r_{k}^{-1}$. The angle-root relation is given in [16]

$$
\Delta \theta_{k}=C_{k} \Im\left[\frac{\Delta r_{k}}{r_{k}}\right]
$$

where $C_{k}$ is in general (see Appendix C)

$$
C_{k}=\frac{\lambda_{e}}{2 \pi d \cos \theta_{k}}
$$

Then

$$
\Delta \theta_{k}=C_{k} \frac{-\Im\left[\mathbf{a}\left(r_{k}^{-1}\right)^{T} \Delta \mathbf{U}_{0} \mathbf{W} \mathbf{U}_{0}^{H} \mathbf{a}^{(1)}\left(r_{k}\right)\right]}{A r_{k} G\left(r_{k}\right)} .
$$

Substitute (3.15) into (5.23) to obtain

$$
\Delta \theta_{k}=C_{k} \frac{\Im\left[\mathbf{a}\left(r_{k}^{-1}\right)^{T} \mathrm{U}_{\imath} \Sigma_{s}^{-1} \mathbf{V}_{t}^{H} \mathbf{N}^{H} \mathrm{U}_{0} \mathbf{W U}_{0}^{H} \mathbf{a}^{(1)}\left(r_{k}\right)\right]}{A r_{k} G\left(r_{k}\right)}
$$

If we define

$$
\alpha_{k}=\mathbf{V}_{,} \boldsymbol{\Sigma}_{s}^{-1} \mathbf{U}_{s}^{H} \mathrm{a}\left(r_{k}\right)
$$


and

$$
\beta_{k}=\mathbf{U}_{o} \mathbf{W} \mathbf{U}_{o}^{H} \mathbf{a}^{(1)}\left(r_{k}\right)
$$

then (5.24) can be simplified as

$$
\Delta \theta_{k}=C_{k} \frac{\Im\left[\alpha_{k}^{H} \mathrm{~N}^{H} \beta_{k}\right]}{A r_{k} G\left(r_{k}\right)}
$$

\subsection{Statistical Performance}

Since we have used a first-order perturbation analysis, the predicted bias of an estimated direction of arrival is

$$
\begin{aligned}
E\left(\Delta \theta_{k}\right) & =E\left[C_{k} \frac{\Im\left(\alpha_{k}^{H} \mathbf{N}^{H} \beta_{k}\right)}{A r_{k} G\left(r_{k}\right)}\right] \\
& =C_{k} \frac{\Im\left[\alpha_{k}^{H} E\left(\mathbf{N}^{H}\right) \beta_{k}\right]}{A r_{k} G\left(r_{k}\right)} \\
& =0 .
\end{aligned}
$$

The mean-squared error of an estimated direction of arrival by (5.25) is

$$
\begin{aligned}
E\left(\Delta \theta_{k}\right)^{2} & =C_{k}^{2} E\left[\Im\left(\frac{\Delta r_{k}}{r_{k}}\right)\right]^{2} \\
& =C_{k}^{2} E\left[\frac{\Im\left(\alpha_{k}^{H} N^{H} \beta_{k}\right)}{A r_{k} G\left(r_{k}\right)}\right]^{2} \\
& =\frac{C_{k}^{2}}{2} \frac{E\left|\alpha_{k}^{H} N^{H} \beta_{k}\right|^{2}}{A^{2}\left|r_{k}\right|^{2}\left|G\left(r_{k}\right)\right|^{2}} \\
& =\frac{C_{k}^{2}}{2} \frac{\left\|\alpha_{k}||^{2}\right\| \beta_{k} \|^{2} \sigma_{n}^{2}}{A^{2}\left|G\left(r_{k}\right)\right|^{2}} .
\end{aligned}
$$

Notice that $\left|r_{k}\right|^{2}=1$ since the noise-free signal roots are on unit-circle.

Min-Norm (RMN): the weighting matrix $\mathbf{W}=\overline{c c}^{H}$ and $A=1$, so

$$
\left\|\beta_{k}\right\|^{2}=\|\bar{c}\|^{2} \| \bar{c}\left[\mathbf{a}^{(1)}\left(r_{k}^{-1}\right)^{T} \mathbf{U}_{o} \bar{c}\left\|^{2}=\right\| \bar{c} \|^{2} A\left|G\left(r_{k}\right)\right|\right.
$$

where the last equality is obtained by using L'Hospital's rule. Then

$$
E\left(\Delta \theta_{k}\right)_{R M N}^{2}=\frac{C_{k}^{2}}{2} \frac{\|\varepsilon\|^{2}\left\|\alpha_{k}\right\|^{2} \sigma_{n}^{2}}{\left|G\left(r_{k}\right)\right|}
$$




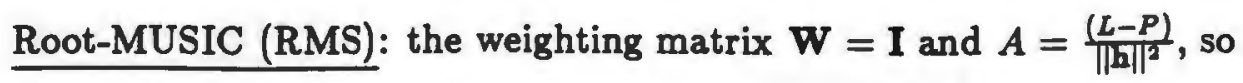

$$
\left\|\beta_{k}\right\|^{2}=\left\|a^{(1)}\left(r_{k}^{-1}\right)^{T} U_{o}\right\|^{2}=A\left|G\left(r_{k}\right)\right|
$$

then

$$
E\left(\Delta \theta_{k}\right)_{R M S}^{2}=\frac{C_{k}^{2}}{2} \frac{\|\mathbf{h}\|^{2}\left\|\alpha_{k}\right\|^{2} \sigma_{n}^{2}}{(L-P)\left|G\left(r_{k}\right)\right|}
$$

\subsection{Relation to Extrema-Searching Algorithms}

As shown above, using L'Hospital's rule, we have (5.28) and (5.30). Then the meansquared error expressions of MUSIC and Min-Norm (5.29) (5.31) can be further written as

$$
E\left(\Delta \theta_{k}\right)_{R M N}^{2}=\frac{C_{k}^{2}}{2} \frac{\|\mathbf{c}\|^{2}\left\|\alpha_{k}\right\|^{2} \sigma_{n}^{2}}{\left|\mathbf{a}^{(1)}\left(r_{k}\right) \mathbf{U}_{o} \mathbf{c}\right|^{2}}
$$

and

$$
E\left(\Delta \theta_{k}\right)_{R M S}^{2}=\frac{C_{k}^{2}}{2} \frac{\left\|\alpha_{k}\right\|^{2} \sigma_{n}^{2}}{\left\|\mathbf{a}^{(1)}\left(r_{k}\right) \mathbf{U}_{0}\right\|^{2}}
$$

These expressions are identical to the mean-squared error expressions derived for extrema-searching algorithms when they are applied to the uniform line array. Therefore the mean-squared error ratio holds for polynomial-rooting approach

$$
\frac{E\left(\Delta \theta_{k}^{2}\right)_{R M N}}{E\left(\Delta \theta_{k}^{2}\right)_{R M S}} \geq 1
$$

\subsection{Mean-Squared Error of Root Perturbation}

From the previous two sections, we can get

$$
E\left(\Delta r_{k}\right)^{2}=\frac{\left\|\alpha_{k}\right\|^{2}\left\|\beta_{k}\right\| \sigma_{n}^{2}}{A^{2}\left|G\left(r_{k}\right)\right|^{2}}
$$

This is from a derivation where $\Delta r_{k}$ is linear in the perturbation of orthogonalsubspace, and thus linear in the zero-mean observation noise. Under such an assumption, (5.34) gives us the mean-squared error of estimated roots $E\left(\Delta r_{k}\right)^{2}$.

$$
E\left(\Delta r_{k}\right)^{2}=\frac{\left\|\alpha_{k}\right\|^{2}\left\|\beta_{k}\right\| \sigma_{n}^{2}}{A^{2}\left|G\left(r_{k}\right)\right|^{2}}
$$


$\underline{\text { Min-Norm (RMN): }}$

$$
E\left(\Delta r_{k}\right)_{R M N}^{2}=\frac{\|\varepsilon\|^{2}\left\|\alpha_{k}\right\|^{2} \sigma_{n}^{2}}{G\left(r_{k}\right)}
$$

Root-MUSIC (RMS):

$$
E\left(\Delta r_{k}\right)_{R M S}^{2}=\frac{\|\mathbf{h}\|^{2}\left\|\alpha_{k}\right\|^{2} \sigma_{n}^{2}}{(L-P) G\left(r_{k}\right)}
$$

However, when the mean-squared error of root estimates is of interest, we need to consider bias. The zero-mean assumption of root estimation error should be avoided. We now present an analysis of the mean-squared error of root estimation error (including the implied contribution of bias squared) of the spectral polynomial (not its derivative). We use similar steps to those used in analyzing the meansquared error of the root estimation. The major difference is we are now dealing with the spectral polynomial itself, instead of dealing with the derivative of the spectral polynomial as we did in the mean-squared error analysis of the angle of the roots.

Step 1. Perturbation of coefficients of spectral polynomial

$$
\begin{aligned}
P\left(z, \tilde{\mathbf{U}}_{0}\right) & =\mathbf{a}\left(z^{-1}\right)^{T} \tilde{\mathbf{U}}_{0} \tilde{\mathbf{W}}_{o}^{H} \mathbf{a}(z) \\
& =\mathbf{a}\left(z^{-1}\right)^{T}\left(\mathbf{U}_{0}+\Delta \mathbf{U}_{0}\right)(\mathbf{W}+\Delta \mathbf{W})\left(\mathbf{U}_{0}+\Delta \mathbf{U}_{0}\right)^{H} \mathbf{a}(z) .
\end{aligned}
$$

Expand (5.38), and notice that all the terms having the factor $a\left(z^{-1}\right)^{T} U_{0}$ or its hermitian conjugate become zero when evaluated at signal roots $r_{k}$. The result is

$$
\left.P\left(z, \tilde{\mathbf{U}}_{0}\right)\right|_{z=r_{k}}=\mathbf{a}\left(r_{k}^{-1}\right)^{T}\left(\Delta \mathbf{U}_{0} \mathbf{W} \Delta \mathbf{U}_{0}^{H}\right) \mathbf{a}\left(r_{k}\right)+\mathbf{a}\left(r_{k}^{-1}\right)^{T}\left(\Delta \mathbf{U}_{0} \Delta \mathbf{W} \Delta \mathbf{U}_{o}^{H}\right) \mathbf{a}\left(r_{k}\right) .
$$

Here we keep the second-order perturbation terms since we are dealing with the "spectral" function, and we neglect the higher-order perturbation term (for RootMUSIC, this term is zero anyway). The result is

$$
\left.P\left(z, \tilde{\mathbf{U}}_{o}\right)\right|_{s=r_{k}}=\mathbf{a}\left(r_{k}^{-1}\right)^{\mathbf{T}}\left(\Delta \mathbf{U}_{0} \mathbf{W} \Delta \mathbf{U}_{o}^{H}\right) \mathbf{a}\left(r_{k}\right) .
$$


Step 2. Perturbation of signal-roots of spectral polynomial

$$
\begin{aligned}
P(z, \tilde{r}) & =A \prod_{i=1}^{L-1}\left(1-\tilde{r}_{i} z^{-1}\right)\left(1-\tilde{r}_{i}^{*} z\right) \\
& =A \prod_{i=1}^{L-1}\left[1-\left(r_{i}+\Delta r_{i}\right) z^{-1}\right]\left[1-\left(r_{i}+\Delta r_{i}\right)^{*} z\right] .
\end{aligned}
$$

Evaluate this function at the signal-roots $r_{k}$ and neglect the higher order terms. We then have

$$
\begin{aligned}
\left.P(z, \tilde{r})\right|_{s=r_{k}} & =A \prod_{j \neq k}^{L-1}\left(1-r_{k} r_{j}^{-1}\right)\left(1-r_{k}^{*} r_{j}\right)\left|\Delta r_{k}\right|^{2} \\
& =A G\left(r_{k}\right)\left|\Delta r_{k}\right|^{2} \text { for } i=1, \ldots, P .
\end{aligned}
$$

Step 3. Equating $\left.P\left(z, \tilde{\mathbf{U}}_{o}\right)\right|_{s=r_{k}}$ and $\left.P(z, \tilde{r})\right|_{z=r_{k}}$, then we obtain

$$
\left|\Delta r_{k}\right|^{2}=\frac{\mathrm{a}\left(r_{k}^{-1}\right)^{T}\left(\Delta \mathrm{U}_{0} \mathbf{W} \Delta \mathrm{U}_{o}^{\nexists}\right) \mathrm{a}\left(r_{k}\right)}{A G\left(r_{k}\right)}
$$

Substitute (3.15) in (5.43) and take the expectation

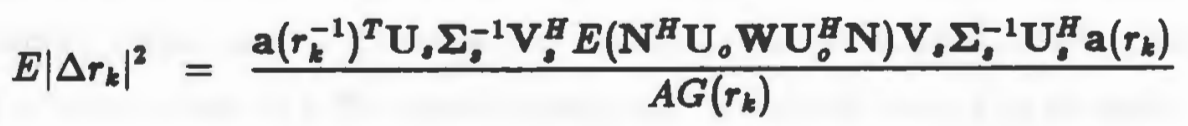

$$
\begin{aligned}
& =\frac{\alpha_{k}^{H} E\left(\mathbf{N}^{H} \mathrm{U}_{0} \mathbf{W} \mathbf{U}_{0}^{H} \mathbf{N}\right) \alpha_{k}}{A G\left(r_{k}\right)}
\end{aligned}
$$

where

$$
\alpha_{k}=\mathbf{V}, \Sigma_{\bullet}^{-1} \mathbf{U}_{\bullet}^{H} \mathbf{a}\left(r_{k}\right) \text {. }
$$

MUSIC(RMN): the weighting matrix $\mathbf{W}=I$ and $A=\frac{(L-P)}{\|\mathrm{h}\|^{2}}$, so

$$
\begin{aligned}
& E\left(\mathbf{N}^{H} \mathbf{U}_{o} \mathbf{W} \mathbf{U}_{o}^{H} \mathbf{N}\right)=(L-P) \sigma_{n}^{2} \\
& E\left|\Delta r_{k}\right|_{R M N}^{2}=\frac{(L-P)\left\|\alpha_{k}\right\|^{2}|| \mathbf{h} \|^{2}}{(L-P) G\left(r_{k}\right)} \sigma_{n}^{2} \\
&=\frac{\|\mathbf{h}\|^{2}\left\|\alpha_{k}\right\|^{2} \sigma_{n}^{2}}{G\left(r_{k}\right)} .
\end{aligned}
$$


Min-Norm(RMS): the weighting matrix $\mathbf{W}=c \bar{c}^{H}$ and $A=1$, so

$$
\begin{aligned}
& E\left(\mathrm{~N}^{H} \mathrm{U}_{0} \mathrm{WU}_{0}^{H} \mathrm{~N}\right)=\|\mathrm{c}\|^{2} \sigma_{n}^{2} \\
& E\left|\Delta \mathrm{r}_{k}\right|_{R M S}^{2}=\frac{\|\mathrm{c}\|^{2}\left\|\alpha_{k}\right\|^{2} \sigma_{n}^{2}}{G\left(r_{k}\right)}
\end{aligned}
$$

Comparing the (5.37) and (5.45), we find that $E\left(\left|\Delta r_{k}\right|\right)^{2} / C_{k}^{2}$ is $L-P$ time larger than $E\left(\Delta \theta_{k}\right)^{2}$ for MUSIC which indicates a large bias exists in the radial direction as verified in simulations. The radial nature of the error makes the extrema in the spatial spectrum less distinct. This renders procedures that examine extrema using a search procedure less attractive. Notice that (5.36) and (5.46) are identical, which indicates the Min-Norm root estimates are unbiased.

\subsection{Numerical Examples}

A twenty-element uniform line array (with $d=\lambda_{c} / 2$ ) is used as shown in Figure 5.1. Angles of arrival signals are measured with respect to the normal of the array. Two sources are considered at 0.2 and 0.5 radians. Twenty snapshots of array data were used to estimate the directions of arrival for a given trial. Data matrices as in (3.1) of dimension $20 \times 20$ were formed, and 500 trials were run at each value of SNR.

Figures 5.2 and 5.3 show the standard deviation of the estimated directions versus SNR of Min-Norm (forward-only), MUSIC, and C-R bound (from top to bottom, respectively). The the measured mean-squared error (shown with an $\star$ ) agrees well with predicted mean-squared error (shown by the lines). Again, our analysis starts from a high-SNR assumption, but the simulation results show that the analytical expressions give accurate results over a wide range of SNR. We also tried the Min-Norm algorithm using a forward-backward data matrix (instead of (3.1)) and then its performance was improved.

Figure 5.4 and 5.5 show the scattergram of the signal roots of Min-Norm and Root-MUSIC algorithms. 


\begin{tabular}{|c|c|c|}
\hline & RMN & RMS \\
\hline $\mid \alpha_{k} \|^{2}$ & $\mid \mathbf{a}\left(r_{k}^{-1}\right)^{T} \mathbf{U}_{i} \Sigma_{a}^{-1} \mathbf{V}_{a} \|^{2}$ & $\left|\mathbf{a}^{(1)}\left(r_{k}^{-1}\right)^{T} U_{o}\left\|^{2}|| c \mid\right\|^{2}\right.$ \\
\hline$\left\|\beta_{k}\right\|^{2}$ & $\left\|\mathbf{a}\left(r_{k}^{-1}\right)^{T} \mathbf{U}, \Sigma_{0}^{-1} \mathbf{V},\right\|^{2}$ & $\left\|\mathbf{a}^{(1)}\left(r_{k}^{-1}\right)^{T} \mathrm{U}_{o}\right\|^{2}$ \\
\hline$\overline{A G\left(r_{k}\right)}$ & $\prod_{\substack{j \neq k \\
j=1}}^{L-1}\left|\left(1-r_{j} r_{k}^{-1}\right)\right|^{2}$ & $\frac{\|h\|^{2}}{L-P} \prod_{\substack{j \neq k \\
j=1}}^{L-1}\left|\left(1-r_{j} r_{k}^{-1}\right)\right|^{2}$ \\
\hline
\end{tabular}

Table 5.1: Parameters of mean-squared error for Polynomial Rooting Algorithms

$s_{\mathbf{k}}$

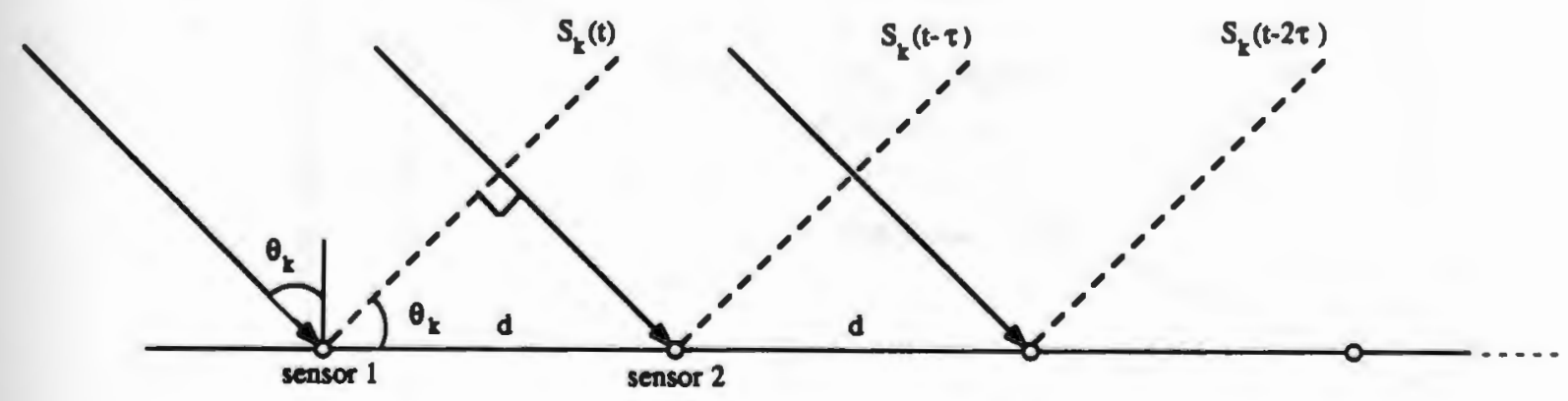

Figure 5.1: Uniform line array 


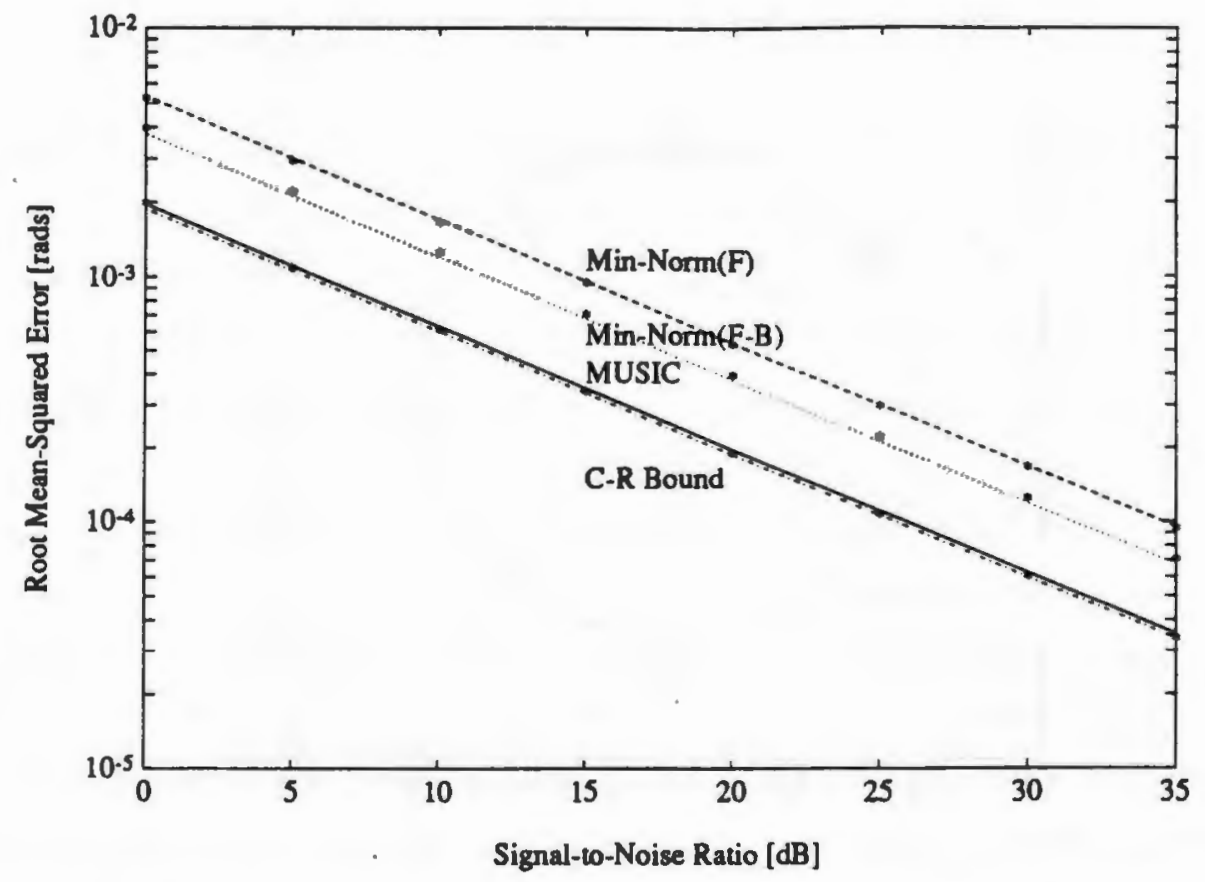

Figure 5.2: RMSE vs. SNR for Min-Norm and Root-MUSIC at source 1 (.2 rads)

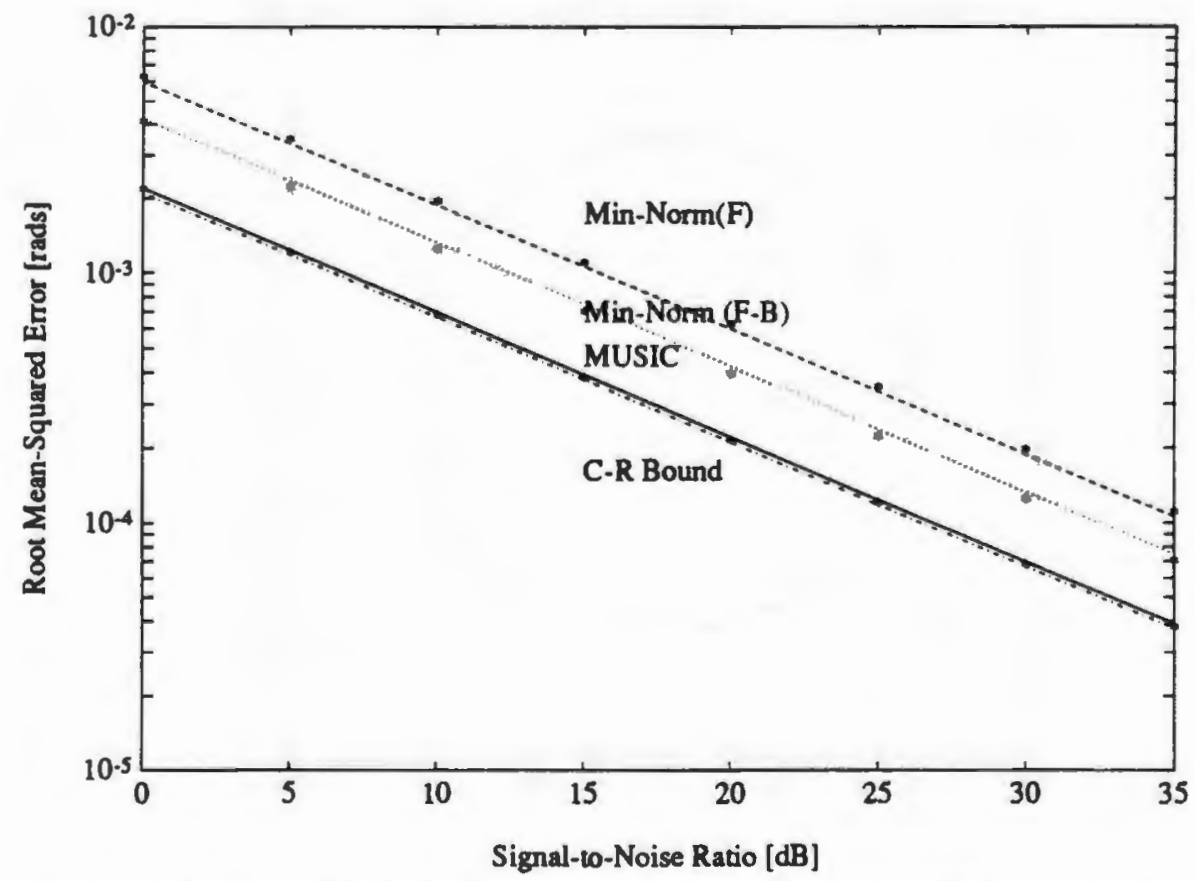

Figure 5.3: RMSE vs. SNR for Min-Norm and Root-MUSIC at source 2 (.5 rads) 


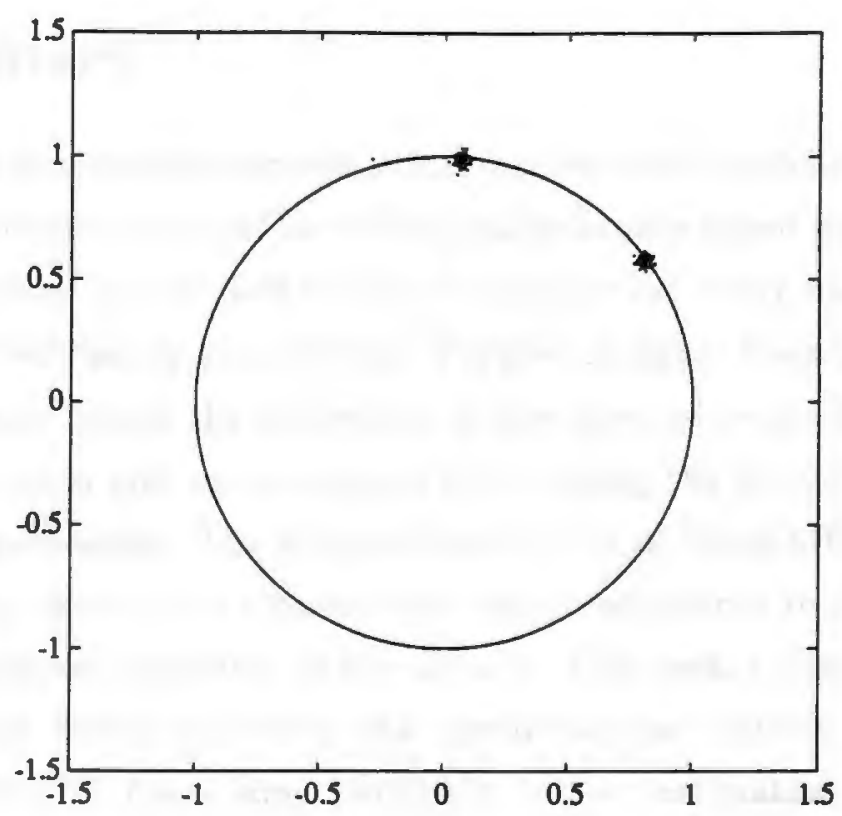

Figure 5.4: Scattergram of Estimated Roots for Min-Norm

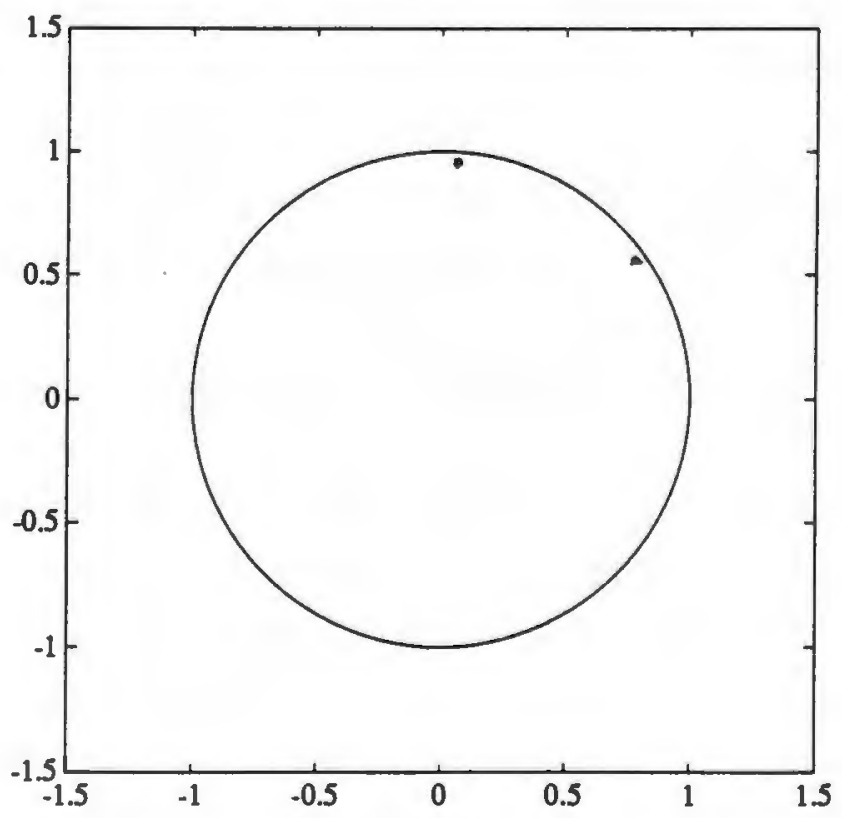

Figure 5.5: Scattergram of Estimated Roots for Root-MUSIC 


\subsection{Summary}

In this chapter, a non-asymptotic statistical performance analysis using matrix approximation to a common model for orthogonal-subspace based polynomial-rooting methods (Min-Norm and Root-MUSIC) of uniform line array has been developed analytically and verified by simulations. Parallel analyses have been made of the mean-squared error (using the derivative of the spectral polynomial) of the estimated signal direction and mean-squared error (using the spectral polynomial) of the estimated signal-roots. The mean-squared error of Root-MUSIC estimates of the DOA's is very close to the Cramer-Rao Bound compared to the mean-squared error of the Min-Norm estimates of the DOA's. This means that Root-MUSIC is preferable in DOA estimation when the signal-to-noise ratio is above threshold. But since Root-MUSIC has a large radial bias in root estimation while Min-Norm is unbiased in root estimation, so Min-Norm is preferable in root estimation or damped sinusoid estimation of time series. 


\section{Chapter 6}

\section{Performance of Matrix Shifting Algorithms}

\subsection{Introduction}

After the extensive research and application of orthogonal subspace methods (Pisarenko, MUSIC and Min-Norm), several signal-subspace based algorithms have been developed more recently.

State-Space Realization (sometimes called Toeplitz Approximation Method and Direct Data Approximation) was first suggested by Kung, Arun and Rao in 1983 $[29,30]$, and further studied by Rao $[64,65,10]$, separately by Foka $[31,32]$ and by Le Cadre [66]. This approach applies the stochastic realization technique to direction of arrival estimation. The property of minimum roundoff noise and coefficient sensitivity of state-space structures was summarized by Jackson for filter design in [67]. Arun proved that a newly developed state-variable balancing technique has the minimum sensitivity with respect to parameter quantization [68].

Estimation of Signal Parameters via Rotational Invariant Techniques (ESPRIT) was proposed by Paulraj, Roy and Kailath $[33,34,35,36,37,38,39,40,41]$, and also studied by Zoltowski [49]. This approach suggests a shift-invariant array which can be considered as two subarrays. The corresponding sensors in each subarray 
are identical, spaced equally in the same direction so that the second subarray can viewed as a shifted version of the first one. The shift-invariance property is therefore utilized.

The Matrix-Pencil Method was proposed by Ouibrahim, Weiner and Sarkar $[44,45,46]$, and summarized by Hua and Sarkar [47]. It forms two data matrices with a certain relationship between them, and solves the generalized eigenvalue problem for the matrix-pencil by exploiting that relationship. Different matrix pencil methods are obtained by using the delay relation (which results in the same algorithm of ESPRIT), the moving-window relation, or the summation relation [46].

The statistical performance of individual signal subspace algorithms have been analyzed: State-Space Realization $[65,69,70,10]$ and Matrix-Pencil [47]. Bhaskar Rao and Hari $[50,71]$ recently presented an interesting analysis of ESPRIT and TAM but their result is based on an asymptotic argument valid only for large data records.

In general, signal-subspace based algorithms utilize a shift-invariant structure of the signal-subspace. So our analysis of performance is based on the signal-subspace perturbation we derived in Chapter 3. Recall that the derivation assumes finite measurements. We begin our analysis of signal subspace methods by establishing a common model of shift invariance.

\subsection{Common Model}

Define a transition equation

$$
\mathbf{A F}=\mathbf{B} \text {. }
$$

The transition matrix is solved as

$$
\mathbf{F}=\mathbf{A}^{\dagger} \mathbf{B} \text {. }
$$

State-Space Realization

$$
\mathbf{A}=\mathbf{O}^{\dagger} \text { and } \mathbf{B}=\mathbf{O}^{\downarrow}
$$




\section{$\underline{\text { ESPRIT }}$}

$$
\mathbf{A}=\mathbf{U}_{s x}^{H} \mathbf{U}_{s x} \text { and } \mathbf{B}=\mathbf{U}_{s x}^{H} \mathbf{U}_{s x} .
$$

If the transition matrix is defined as

$$
\mathbf{F A}=\mathbf{B}
$$

the solution is

$$
\mathbf{F}=\mathbf{B A}^{\prime}
$$

where the superscript $\sharp$ stands for right pseudo-inverse.

\subsection{Perturbation of the Angle Estimates}

In analyzing the perturbation of estimated DOA, we adopt the following three steps:

1. Perturbation of the transition Matrix F

2. Perturbation of the eigenvalues of transition matrix

3. Perturbation of the angles of the eigenvalues

Step 1. Perturbation of the transition Matrix F.

With the presence of observation noise, the transition equation is

$$
\tilde{\mathbf{A}} \tilde{\mathbf{F}}=\tilde{\mathbf{B}} \text {. }
$$

If we define

$$
\begin{aligned}
\tilde{\mathbf{A}} & =\mathbf{A}+\Delta \mathbf{A} \\
\tilde{\mathbf{B}} & =\mathbf{B}+\Delta \mathbf{B} \\
\tilde{\mathbf{F}} & =\mathbf{F}+\Delta \mathbf{F}
\end{aligned}
$$

then

$$
(\mathbf{A}+\Delta \mathbf{A})(\mathbf{F}+\Delta \mathbf{F})=(\mathbf{B}+\Delta \mathbf{B}),
$$


which can be expanded and re-arranged as

$$
\mathbf{A F}+\mathbf{A} \Delta \mathbf{F}+\Delta \mathbf{A F}+\Delta \mathbf{A} \Delta \mathbf{F}=\mathbf{B}+\Delta \mathbf{B}
$$

We can cancel $\mathbf{A F}$ and $\mathbf{B}$, and neglect the second-order perturbation term $\Delta \mathbf{A} \Delta \mathbf{F}$ to obtain

$$
\mathbf{A} \Delta \mathbf{F}+\Delta \mathbf{A F}=\Delta \mathbf{B} .
$$

Finally, we can solve for $\Delta F$ as

$$
\Delta \mathbf{F}=\mathbf{A}^{\dagger}(\Delta \mathbf{B}-\Delta \mathbf{A F})
$$

where the superscript $\dagger$ denotes left pseudo-inverse.

If the transition equation is

$$
\mathbf{F A}=\mathbf{B}
$$

a similar result can be derived:

$$
\Delta \mathbf{F}=(\Delta \mathbf{B}-\mathbf{F} \Delta \mathbf{A}) \mathbf{A}^{\prime}
$$

where the superscript $\sharp$ denotes right pseudo-inverse.

State-Space Realization

The transition equation in the noise-free case for State-Space Realization is

$$
\mathbf{O}^{\dagger} \mathbf{F}=\mathbf{O}^{\dagger}
$$

and the transition matrix is

$$
\mathbf{F}=\mathbf{O}^{\dagger} \mathbf{O}^{\dagger}
$$

Therefore the perturbation of the transition matrix is

$$
\Delta \mathbf{F}=\mathbf{O}^{\dagger}\left(\Delta \mathbf{O}^{\dagger}-\Delta \mathbf{O}^{\dagger}\right)
$$

where from (2.35),

$$
\mathbf{O}=\mathbf{U}, \mathbf{\Sigma}^{\frac{1}{2}} .
$$


Notice that since the column vectors of $O$ are linear combinations of the columnvectors of the left-most matrix $U_{0}$, the up- and down- shift of $O$ are obtained by an up- and down- shift of $U$.

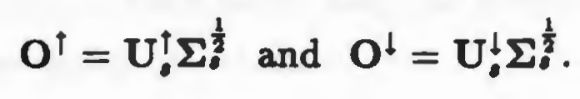

Also

$$
\Delta \mathbf{O}=\mathbf{U}, \mathbf{\Sigma}^{\frac{1}{2}}
$$

and so

$$
\Delta \mathbf{O}^{\dagger}=\Delta \mathbf{U}_{0}^{\dagger} \Sigma_{i}^{\frac{1}{2}} \text { and } \Delta \mathbf{O}^{\downarrow}=\Delta \mathbf{U}_{\bullet}^{\dagger} \Sigma_{?}^{\frac{1}{2}}
$$

In addition

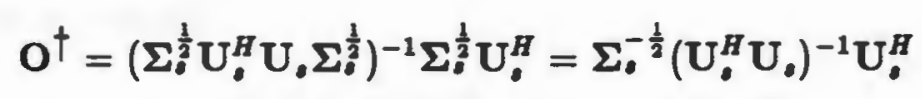

with

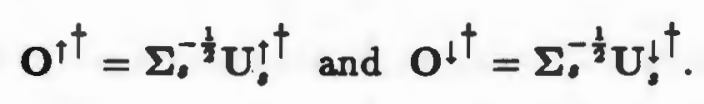

Substituting into (6.9) yields

$$
\Delta \mathbf{F}=\boldsymbol{\Sigma}^{-\frac{1}{2}} \mathbf{U}^{\dagger}\left(\Delta \mathbf{U}^{\dagger} \mathbf{\Sigma}^{\frac{1}{2}}-\Delta \mathbf{U}_{\bullet}^{!} \boldsymbol{\Sigma}_{?}^{\frac{1}{2}} \mathbf{F}\right) .
$$

ESPRIT

The transition matrix of ESPRIT in the noise-free case is obtained from

$$
\mathbf{U}_{s z}^{H} \mathbf{U}_{s x} \mathbf{F}=\mathbf{U}_{s x}^{H} \mathbf{U}_{s x}
$$

and in noisy case, from

$$
\tilde{\mathbf{U}}_{s z}^{H} \tilde{\mathbf{U}}_{s z} \tilde{\mathbf{F}}=\tilde{\mathbf{U}}_{s z}^{H} \tilde{\mathbf{U}}_{\theta x} .
$$

We can expand the above equation to obtain

$$
\left(\mathbf{U}_{s z}+\Delta \mathbf{U}_{\theta z}\right)^{H}\left(\mathbf{U}_{\theta z}+\Delta \mathbf{U}_{\theta z}\right)(\mathbf{F}+\Delta \mathbf{F})=\left(\mathbf{U}_{\theta x}+\Delta \mathbf{U}_{\theta x}\right)^{H}\left(\mathbf{U}_{\theta z}+\Delta \mathbf{U}_{\theta z}\right) .
$$

Expand both sides of the above equation yields

$$
\mathbf{U}_{s x}^{H} \mathbf{U}_{s x} \mathbf{F}+\mathbf{U}_{s x}^{H} \mathbf{U}_{s x} \Delta \mathbf{F}+\Delta \mathbf{U}_{s x}^{H} \mathbf{U}_{s z} \mathbf{F}+\mathbf{U}_{s x}^{H} \Delta \mathbf{U}_{s x} F
$$




$$
\begin{aligned}
& +\Delta \mathbf{U}_{s x}^{H} \Delta \mathbf{U}_{e x} \mathbf{F}+\Delta \mathbf{U}_{e x}^{H} \mathbf{U}_{e x} \Delta \mathbf{F}+\mathbf{U}_{s x}^{H} \mathbf{U}_{e z} \Delta \mathbf{F}+\Delta \mathbf{U}_{s z}^{H} \Delta \mathbf{U}_{s z} \Delta \mathbf{F} \\
& =\mathrm{U}_{s x}^{H} \mathrm{U}_{s x}+\mathrm{U}_{s x}^{H} \Delta \mathrm{U}_{\theta x}+\Delta \mathrm{U}_{s x}^{H} \mathrm{U}_{\theta x}+\mathrm{U}_{s x}^{H} \mathrm{U}_{s x} .
\end{aligned}
$$

We can cancel the first terms on both sides, keep the first-order perturbation terms, and drop the higher-order terms to obtain

$$
\mathbf{U}_{s z}^{H} \mathrm{U}_{s x} \Delta \mathbf{F}+\Delta \mathbf{U}_{s z}^{H} \mathbf{U}_{s z} \mathbf{F}+\mathbf{U}_{s x}^{H} \Delta \mathbf{U}_{s z} \mathbf{F}=\mathbf{U}_{s x}^{H} \Delta \mathbf{U}_{s x}+\Delta \mathbf{U}_{s x}^{H} \mathbf{U}_{s x}
$$

which can be re-arranged as

$$
\left(\mathbf{U}_{s x}^{H} \mathbf{U}_{s x}\right) \Delta \mathbf{F}=\mathbf{U}_{s x}^{H}\left(\Delta \mathbf{U}_{s x}-\Delta \mathbf{U}_{s z} \mathbf{F}\right)+\Delta \mathbf{U}_{s x}^{H}\left(\mathbf{U}_{s x}-\mathbf{U}_{s z} \mathbf{F}\right) .
$$

The last term on right hand-side is zero because it satisfies the noise free transition equation, and so we obtain

$$
\Delta \mathbf{F}=\left(\mathbf{U}_{s z}^{H} \mathbf{U}_{s x}\right)^{-1} \mathbf{U}_{s z}^{H}\left(\Delta \mathbf{U}_{s z}-\Delta \mathbf{U}_{s z} \mathbf{F}\right) .
$$

Step 2. Perturbation of the eigenvalues of the transition matrix.

The perturbations of an eigenvalue and corresponding eigenvector are given in [72]. The first-order perturbation of an eigenvalue is

$$
\begin{aligned}
\Delta \lambda_{k} & =\mathbf{v}_{k} \Delta \mathbf{F u} \mathbf{u}_{k} \\
& =\mathbf{v}_{k} \mathbf{A}^{\dagger}(\Delta \mathbf{B}-\Delta \mathbf{A F}) \mathbf{u}_{k} \\
& =\mathbf{v}_{k} \mathbf{A}^{\dagger}\left(\Delta \mathbf{B}-\lambda_{k} \Delta \mathbf{A}\right) \mathbf{u}_{k}
\end{aligned}
$$

where the $v_{k}$ and $u_{k}$ are the left- and right- eigenvectors of $F$ associated with $\bar{\lambda}_{k}$. State-Space Realization

The eigenvalue perturbation of (6.9) is

$$
\begin{aligned}
& \Delta \lambda_{k}=\frac{\mathbf{v}_{k}\left(\Sigma_{e}^{-\frac{1}{2}} U_{!}^{\dagger}\left(\Delta U_{!}^{\dagger} \Sigma_{e}^{\frac{1}{2}}-\Delta U_{e}^{!} \Sigma_{s}^{\frac{1}{2}} F\right) \mathbf{u}_{k}\right.}{\bar{\lambda}_{k}}
\end{aligned}
$$

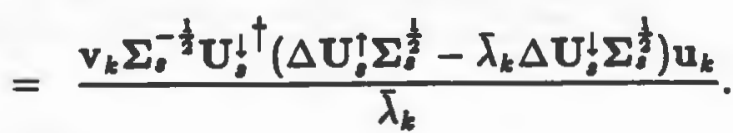

\section{ESPRIT}


The eigenvalue perturbation of $(6.11)$ is

$$
\begin{aligned}
& \Delta \bar{\lambda}_{k}=\frac{\mathbf{v}_{k}\left(\mathbf{U}_{g z}^{H} \mathbf{U}_{g s}\right)^{-1} \mathbf{U}_{g z}^{H}\left(\Delta \mathbf{U}_{g z}-\mathbf{F} \Delta \mathbf{U}_{e s}\right) \mathbf{u}_{k}}{\lambda_{k}} \\
& =\frac{\mathbf{v}_{k}\left(\mathbf{U}_{g x}^{H} \mathbf{U}_{g s}\right)^{-1} \mathbf{U}_{s g}^{H}\left(\Delta \mathbf{U}_{g z}-\bar{\lambda}_{k} \Delta \mathbf{U}_{\theta g}\right) \mathbf{u}_{k}}{\lambda_{k}} .
\end{aligned}
$$

Step 3. Perturbation of the angles of the eigenvalues.

The eigenvalues of the transition matrix are the same as the roots of the parameter polynomial, so the angle-root relationship in Appendix C holds

$$
\begin{aligned}
\Delta \theta_{k} & =C_{k} \Im\left(\frac{\Delta \lambda_{k}}{\bar{\lambda}_{k}}\right) \\
& =C_{k} \Im\left(\frac{\mathbf{v}_{k} \Delta \mathbf{F} \mathbf{u}_{k}}{\bar{\lambda}_{k}}\right) \\
& =C_{k} \Im\left[\frac{\mathbf{v}_{k} \mathbf{A}^{\dagger}(\Delta \mathbf{B}-\Delta \mathbf{A F}) \mathbf{u}_{k}}{\bar{\lambda}_{k}}\right] \\
& =C_{k} \Im\left[\frac{\mathbf{v}_{k} \mathbf{A}^{\dagger}\left(\Delta \mathbf{B}-\bar{\lambda}_{k} \Delta \mathbf{A}\right) \mathbf{u}_{k}}{\bar{\lambda}_{k}}\right]
\end{aligned}
$$

where $C_{k} \stackrel{\text { def }}{=} \frac{\lambda_{c}}{2 \pi d \cos \theta_{k}}$, and $\Im[\cdot]$ is the imaginary part of $[\cdot]$.

State-Space Realization

Substitute

$$
\Delta \mathbf{U}_{\bullet}^{!}=\mathbf{U}_{0}^{\dagger} \mathbf{U}_{0}^{H} \mathbf{N V}, \mathbf{\Sigma}_{0}^{-1} \text { and } \Delta \mathbf{U}^{\dagger}=\mathbf{U}_{0}^{\dagger} \mathbf{U}_{0}^{H} \mathbf{N} \mathbf{V}, \mathbf{\Sigma}_{0}^{-1}
$$

in (6.13) to obtain

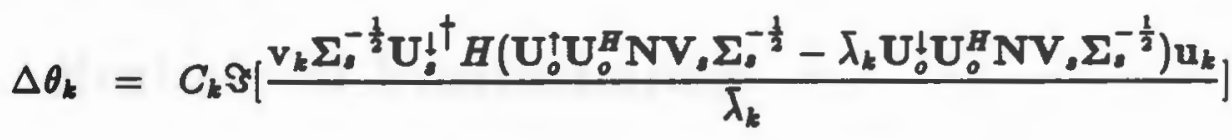

$$
\begin{aligned}
& =C_{k} \Im\left[\frac{\mathbf{v}_{k} \Sigma_{s}^{-\frac{l}{2}} \mathbf{U}_{j}^{\dagger}\left(\mathbf{U}_{o}^{\dagger}-\lambda_{k} \mathbf{U}_{0}^{\dagger}\right) \mathbf{U}_{o}^{H} \mathbf{N V}, \Sigma_{s}^{-\frac{1}{2}} \mathbf{u}_{k}}{\lambda_{k}}\right] \\
& =C_{k} \Im\left[\frac{\alpha_{k}^{H} \mathbf{N} \beta_{k}}{\bar{\lambda}_{k}}\right]
\end{aligned}
$$


where

$$
\alpha_{k}^{H}=\mathbf{v}_{k} \boldsymbol{\Sigma}_{\bullet}^{-\frac{1}{2}} \mathbf{U}_{o}^{\dagger}\left(\mathbf{U}_{o}^{\dagger}-\bar{\lambda}_{k} \mathbf{U}_{o}^{\dagger}\right) \mathbf{U}_{o}^{H}
$$

and

$$
\boldsymbol{\beta}_{\boldsymbol{k}}=\mathrm{V}, \boldsymbol{\Sigma}^{-\frac{1}{3}} \mathbf{u}_{\boldsymbol{k}}
$$

where the $\mathbf{U}_{0}^{\dagger}$ and $\mathbf{U}_{0}^{!}$are defined as the up- and down- shift of $\mathbf{U}_{0}$.

ESPRIT

Substitute

$$
\Delta \mathrm{U}_{s z}=\mathrm{U}_{o z} \mathrm{U}_{0}^{H} \mathrm{NV}, \Sigma_{s}^{-1} \text { and } \Delta \mathrm{U}_{s z}=\mathrm{U}_{o z} \mathrm{~V}_{0}^{H} \mathrm{NV}_{s} \boldsymbol{\Sigma}_{s}^{-1}
$$

in (6.14) to obtain

$$
\begin{aligned}
& \Delta \theta_{k}=C_{k} \Im\left[\frac{\mathbf{v}_{k}\left(\mathrm{U}_{o z}^{H} \mathrm{U}_{o s}\right)^{-1} \mathrm{U}_{g z}^{H}\left(\mathrm{U}_{o z} \mathrm{U}_{o}^{H} \mathrm{NV}, \Sigma_{a}^{-1}-\bar{\lambda}_{k} \mathrm{U}_{o z} \mathrm{U}_{o}^{H} \mathrm{NV}, \Sigma_{e}^{-1}\right) \mathbf{u}_{k}}{\bar{\lambda}_{k}}\right] \\
& =C_{k} \Im\left[\frac{\mathbf{v}_{k}\left(\mathbf{U}_{s z}^{H} \mathrm{U}_{s z}\right)^{-1} \mathbf{U}_{s z}^{H}\left(\mathrm{U}_{o z}-\bar{\lambda}_{k} \mathrm{U}_{o z}\right) \mathbf{U}_{o}^{H} \mathbf{N V}, \Sigma_{s}^{-1} \mathbf{u}_{k}}{\bar{\lambda}_{k}}\right] \\
& =C_{k} \Im\left[\frac{\alpha_{k}^{H} \mathbf{N} \beta_{k}}{\bar{\lambda}_{k}}\right]
\end{aligned}
$$

where

$$
\alpha_{k}^{H}=\mathbf{v}_{k}\left(\mathrm{U}_{e z}^{H} \mathrm{U}_{s z}\right)^{-1} \mathrm{U}_{\theta z}^{H}\left(\mathrm{U}_{o z}-\bar{\lambda}_{k} \mathrm{U}_{o z}\right) \mathrm{U}_{o}^{H}
$$

and

$$
\beta_{k}=\mathrm{V}, \Sigma_{\mathfrak{\jmath}}^{-1} \mathbf{u}_{k}
$$

where the $U_{o x}$ and $U_{o z}$ are defined from the rows in $U_{o}$ corresponding to subarray $\mathrm{X}$ and $\mathrm{Z}$.

\subsection{Statistical Performance}

Under the first-order approximation, the bias of an estimated direction of arrival is

$$
E\left(\Delta \theta_{k}\right)=C_{k} E\left\{\Im\left[\frac{\mathbf{v}_{k} \mathbf{A}^{\dagger}\left(\Delta \mathbf{B}-\bar{\lambda}_{k} \Delta \mathbf{A}\right) \mathbf{u}_{k}}{\lambda_{k}}\right]\right\}
$$




$$
\begin{aligned}
& =C_{k} \frac{\alpha_{k}^{B} E(\mathbf{N}) \beta_{k}}{\lambda_{k}} \\
& =0 .
\end{aligned}
$$

The mean-squared error of an estimated direction of arrival is

$$
\begin{aligned}
E\left(\Delta \theta_{k}\right)^{2} & =C_{k}^{2} E\left\{\Im\left[\frac{\Delta \bar{\lambda}_{k}}{\bar{\lambda}_{k}}\right]\right\}^{2} \\
& =\frac{C_{k}^{2}}{2} E\left|\frac{\Delta \bar{\lambda}_{k}}{\bar{\lambda}_{k}}\right|^{2} \\
& =\frac{C_{k}^{2}}{2} E\left|\frac{\mathbf{v}_{k} \mathbf{A}^{\dagger}\left(\Delta \mathrm{B}-\lambda_{k} \Delta \mathbf{A}\right) \mathbf{u}_{k}}{\lambda_{k}}\right|^{2} \\
& =\frac{C_{k}^{2}}{2} \frac{E\left|\alpha_{k}^{H} \mathbf{N} \beta_{k}\right|^{2}}{\left|\lambda_{k}\right|^{2}} \\
& =\frac{C_{k}^{2}}{2}\left\|\alpha_{k}\right\|^{2}\left\|\beta_{k}\right\|^{2} \sigma_{n}^{2} .
\end{aligned}
$$

Notice that $\left|\lambda_{k}\right|^{2}=1$ because the noise-free signal roots are on the unit circle.

State-Space Realization (SSR)

With $\alpha_{k}$ and $\beta_{k}$ of State-Space Realization

$$
\alpha_{k}^{H}=\mathbf{v}_{k} \Sigma_{s^{-\frac{1}{2}}}^{\mathrm{U}_{0}^{\dagger}}{ }^{\dagger}\left(\mathrm{U}_{0}^{\dagger}-\bar{\lambda}_{k} \mathrm{U}_{0}^{\dagger}\right) \mathrm{U}_{0}^{H}
$$

and

$$
\beta_{k}=V_{s} \Sigma^{-\frac{1}{2}} \mathbf{u}_{k},
$$

the mean-squared error of an estimated direction of arrival is

$$
\begin{aligned}
E\left(\Delta \theta_{k}\right)_{S S R}^{2} & =\frac{C_{k}^{2}}{2}\left\|\alpha_{k}\right\|^{2}\left\|\beta_{k}\right\|^{2} \sigma_{n}^{2} \\
& =\frac{C_{k}^{2} \sigma_{n}^{2}}{2}\|\| \mathbf{v}_{k} \Sigma_{\bullet}^{-\frac{1}{2}} \mathrm{U}_{\bullet}^{\dagger}\left(\mathrm{U}_{0}^{\dagger}-\bar{\lambda}_{k} \mathrm{U}_{0}^{\frac{1}{}}\left\|^{2}\right\| \Sigma_{\bullet}^{-\frac{1}{2}} \mathbf{u}_{k} \|^{2} .\right.
\end{aligned}
$$

ESPRIT (ESP)

With $\alpha_{k}$ and $\beta_{k}$ of ESPRIT

$$
\alpha_{k}^{H}=\mathbf{v}_{k}\left(\mathrm{U}_{s z}^{H} \mathrm{U}_{s z}\right)^{-1} \mathrm{U}_{s z}^{H}\left(\mathrm{U}_{o z}-\bar{\lambda}_{k} \mathrm{U}_{o z}\right) \mathrm{U}_{o}^{H}
$$


and

$$
\beta_{k}=\mathbf{V}, \Sigma_{*}^{-1} \mathbf{u}_{k},
$$

the mean-squared error of an estimated direction of arrival is

$$
\begin{aligned}
E\left(\Delta \theta_{k}\right)_{E P R}^{2} & =\frac{C_{k}^{2}}{2}\left\|\alpha_{k}\right\|^{2}\left\|\beta_{k}\right\|^{2} \sigma_{n}^{2} \\
& =\frac{C_{k}^{2} \sigma_{n}^{2}}{2}\left\|\mathbf{v}_{k}\left(\mathrm{U}_{s z}^{H} \mathrm{U}_{o s}\right)^{-1} \mathrm{U}_{s x}^{H}\left(\mathrm{U}_{o x}-\bar{\lambda}_{k} \mathrm{U}_{o x}\right)\right\|^{2}\left\|\Sigma_{s}^{-1} \mathbf{u}_{k}\right\|^{2} .
\end{aligned}
$$

\begin{tabular}{|c|c|c|}
\hline & SSR & ESP \\
\hline$\left\|\alpha_{k}\right\|^{2}$ & $\left\|\mathbf{v}_{k} \Sigma_{o}^{-\frac{1}{2}} \mathbf{U}_{s}^{\dagger}\left(\mathbf{U}_{o}^{\dagger}-\lambda_{k} \mathbf{U}_{o}^{l}\right) \mathbf{U}_{o}^{H}\right\|^{2}$ & $\left\|\mathbf{v}_{k}\left(\mathbf{U}_{s z}^{H} \mathbf{U}_{o z}\right)^{-1} \mathbf{U}_{s z}^{H}\left(\mathbf{U}_{o x}-\lambda_{k} \mathbf{U}_{o z}\right) \mathbf{U}_{o}^{H}\right\|^{2}$ \\
\hline$\left\|\boldsymbol{\beta}_{k}\right\|^{2}$ & $\left\|\mathbf{V}_{s} \Sigma_{o}^{-\frac{1}{2}} \mathbf{u}_{k}\right\|^{2}$ & $\left\|\mathbf{V}, \Sigma_{s}^{-1} \mathbf{u}_{k}\right\|^{2}$ \\
\hline
\end{tabular}

Table 6.1: Parameters of mean-squared error for Matrix Shifting Algorithms

\subsection{Numerical Examples}

In general, the array geometry of the Matrix Shifting approach requires some type of shift-invariance. For instance, the ESPRIT array shown in Figure 6.1 has a displacement invariance (sensors can be grouped in pairs with identical displacements). In the example in this section, we use the uniform line array with same configuration as in Chapter 5 for the purpose of comparison.

Figure 6.2 shows the scattergram of the eigenvalues of the matrix shifting approaches (no difference between State-Space Realization and ESPRIT).

Figures 6.3 and 6.4 show the standard deviation of the estimated directions versus of SSR, ESPRIT, and C-R bound (from top to bottom, respectively). The simulation results (shown with an $\star$ ) agree closely with theoretical predictions (shown by the lines). Our analysis starts from a high-SNR assumption, but the simulation results show that the analytical expressions give accurate results over a wide range of SNR extending down near the threshold SNR of the algorithms. SSR and 
ESPRIT had virtually identical performance (the middle lines on the graphs). We also tried the Min-Norm algorithm using a forward-backward data matrix (instead of (3.1)) and then its performance was the same as SSR and ESPRIT.

\subsection{Summary}

In this chapter, a non-asymptotic statistical performance using matrix approximation to a common model for signal-subspace based matrix-shifting algorithms (applicable to State-Space Realization, ESPRIT and Matrix-Pencil Method) of an array with shift-invariant geometry has been developed analytically and verified by simulations. The analysis was made in steps of analyzing the perturbations of the transition matrix, its eigenvalues, and finally perturbations of the signal arrival angles. The formula for the perturbation of the signal-subspace derived in chapter 3 was used as the basis for our unified analysis. Tractable mean-squared error formulas of various signal-subspace based methods for DOA estimation have been derived. 


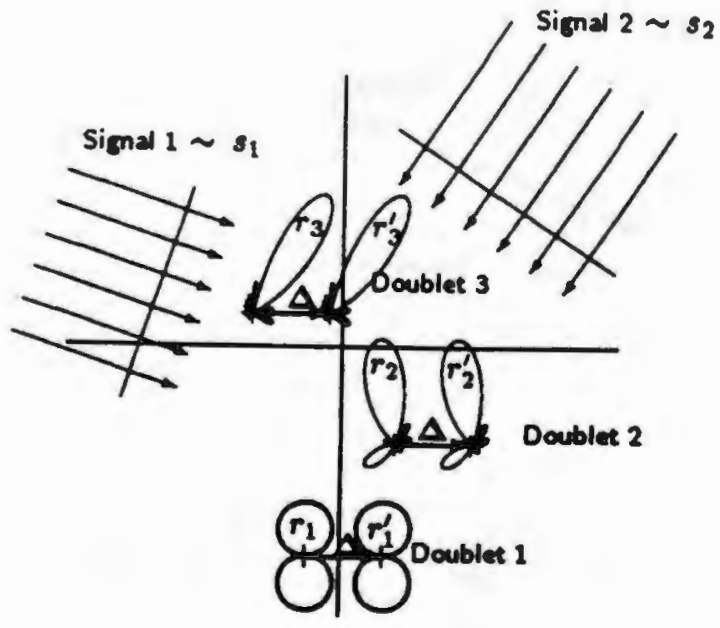

Figure 6.1: ESPRIT Array geometry from [35]

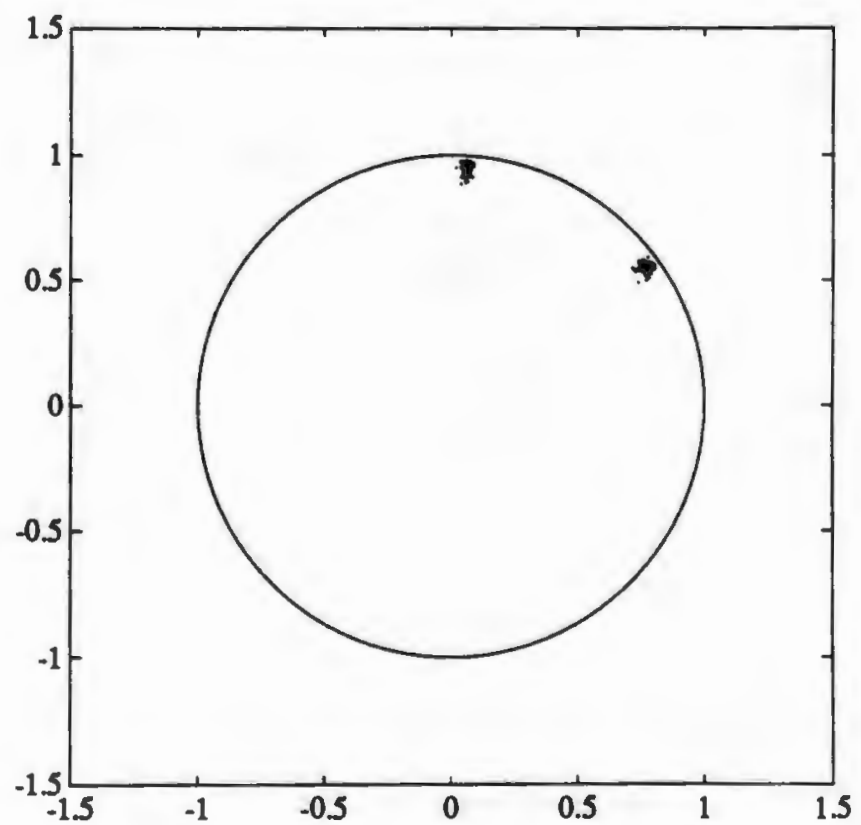

Figure 6.2: Scattergram of Estimated eigenvalues 


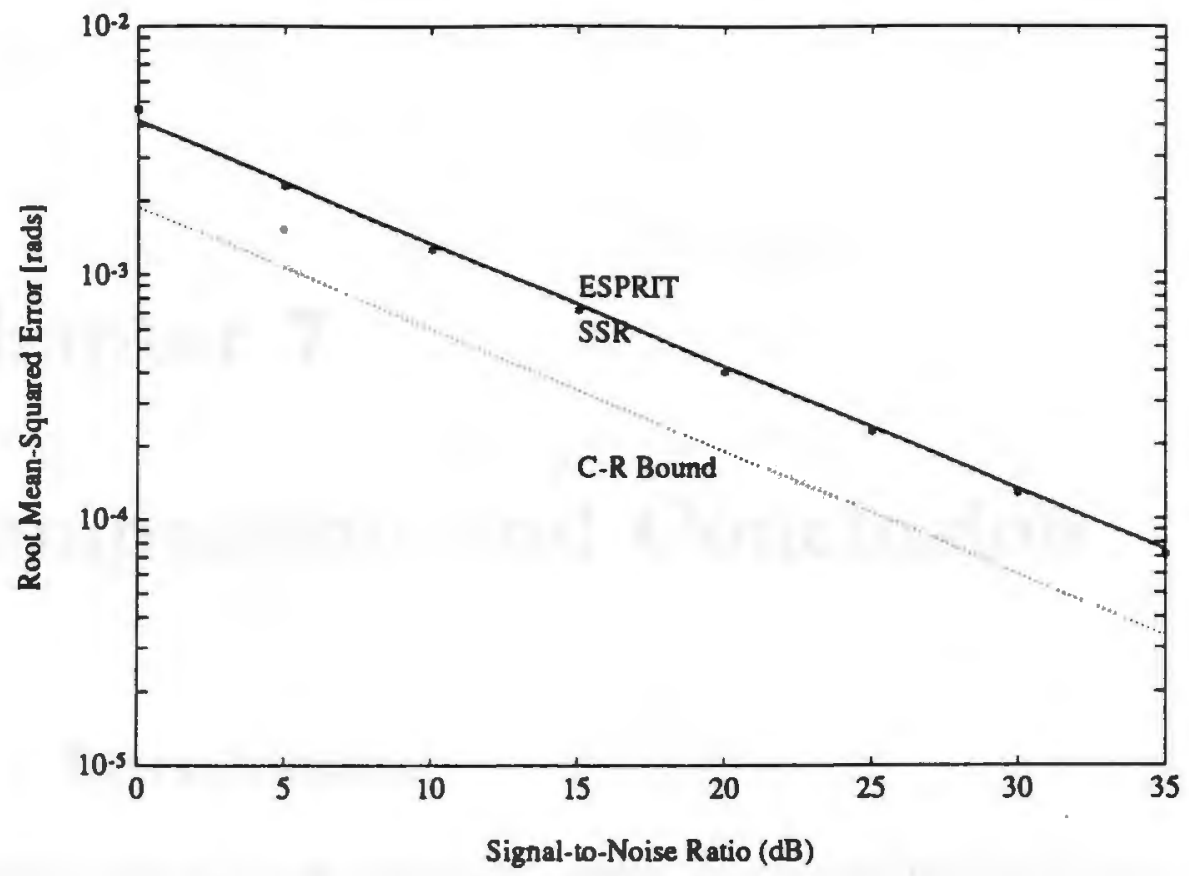

Figure 6.3: RMSE vs. SNR for SSR and ESPRIT at source 1 (.2 rads)

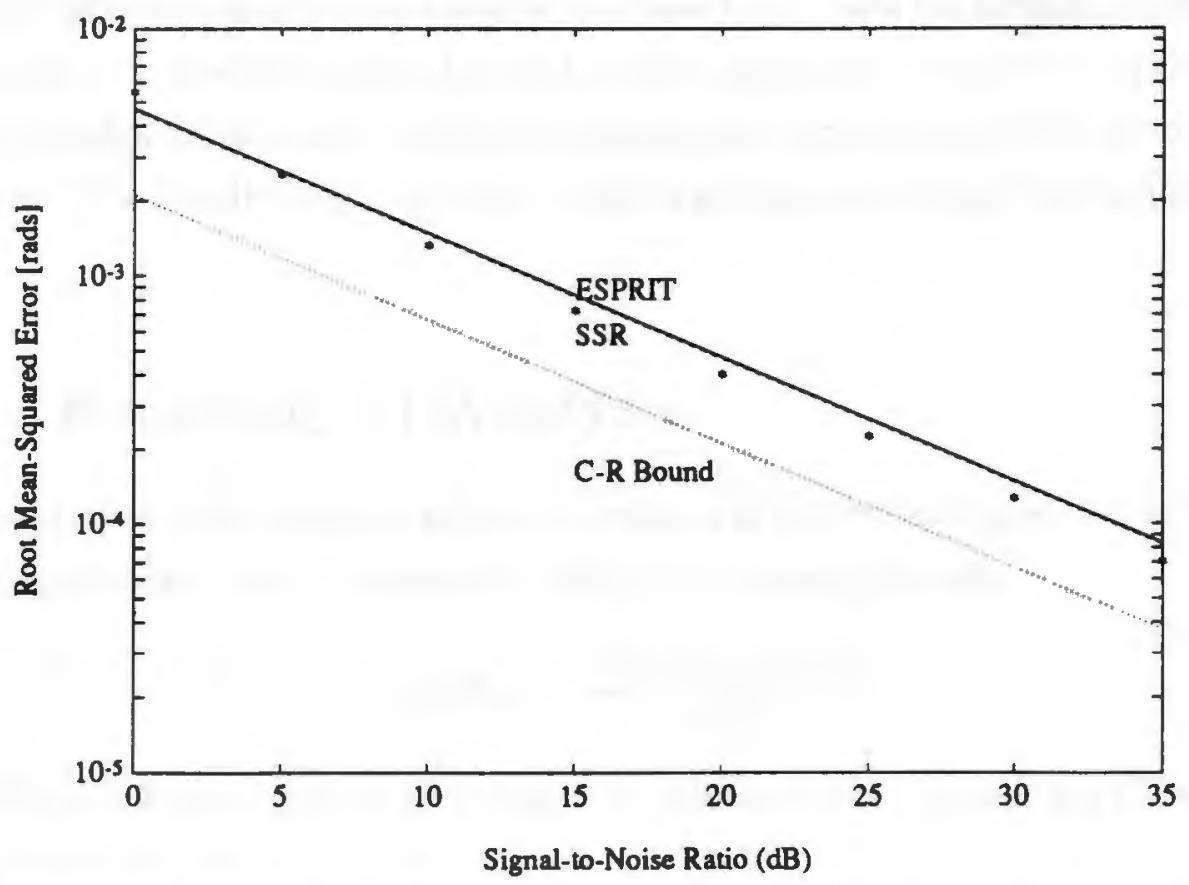

Figure 6.4: RMSE vs. SNR for SSR and ESPRIT at source 2 (.5 rads) 


\section{Chapter 7}

\section{Comparison and Conclusion}

\subsection{Introduction}

We have analyzed three different numerical approaches for direction of arrival estimation based on either the signal or the orthogonal subspaces. We shall, in this chapter, first summarize the analysis [Section 7.1]. Then we present numerical examples of the predicted and observed performance with respect to signal-to-noise ratio, number of sensors, number of snapshots, separation of the source angles [Section 7.2]. Finally, we make the concluding remarks of this dissertation [Section 7.3].

\subsection{Summary of Analysis}

The predicted mean-squared error of direction of arrival estimates for all subspace based algorithms can be summarized by the following formula

$$
E\left(\Delta \theta_{k}\right)^{2}=\frac{C\left\|\alpha_{k}\right\|^{2}\left\|\beta_{k}\right\|^{2} \sigma_{n}^{2}}{2 \gamma_{k}^{2}}
$$

For different algorithms, one just needs to substitute the appropriate $C, \alpha_{k}, \beta_{k}$ and $\gamma_{k}$ as shown below 
- $\underline{\text { MUSIC }}$ (SMS)

$$
\begin{aligned}
C & =1 \\
\alpha_{k} & =\left\|\mathbf{a}\left(\theta_{k}\right)^{H} \mathbf{U}_{0} \Sigma_{a}^{-1} \mathbf{V}_{0}\right\|^{2} \\
\beta_{k} & =\left\|\mathbf{a}^{(1)}\left(\theta_{k}\right)^{H} \mathbf{U}_{o}\right\|^{2} \\
\gamma_{k} & =\left\|a^{(1)}\left(\theta_{k}\right)^{H} \mathbf{U}_{o}\right\|^{2} .
\end{aligned}
$$

- $\underline{\text { Min-Norm (searching) (SMN) }}$

$$
\begin{aligned}
C & =1 \\
\alpha_{k} & =\left\|\mathbf{a}\left(\theta_{k}\right)^{H} \mathbf{U}_{\iota} \Sigma_{\bullet}^{-1} \mathbf{V}_{\iota}\right\|^{2} \\
\beta_{k} & =\left\|\mathbf{a}^{(1)}\left(\theta_{k}\right)^{H} \mathbf{U}_{0}\right\|^{2}\|\mathbf{c}\|^{2} \\
\gamma_{k} & =\left\|\mathbf{a}^{(1)}\left(\theta_{k}\right)^{H} \mathbf{U}_{0} \mathbf{c}\right\|^{2} .
\end{aligned}
$$

- Min-Norm (RMN)

$$
\begin{aligned}
C & =\left(\frac{\lambda_{c}}{2 \pi d \cos \theta_{k}}\right)^{2} \\
\alpha_{k} & =\left\|\mathbf{a}\left(r_{k}\right)^{H} \mathbf{U}_{,} \Sigma_{s}^{-1} \mathbf{V}_{\jmath}\right\|^{2} \\
\beta_{k} & =\left\|\mathbf{a}^{(1)}\left(r_{k}\right)^{H} \mathbf{U}_{o}\right\|^{2}\|\mathbf{c}\|^{2} \\
\gamma_{k} & =\prod_{\substack{j \neq k \\
j=1}}^{L-1}\left|\left(1-r_{j} r_{k}^{-1}\right)\right|^{2} .
\end{aligned}
$$

- $\underline{\text { Root-MUSIC (RMS) }}$

$$
\begin{aligned}
C & =\left(\frac{\lambda_{c}}{2 \pi d \cos \theta_{k}}\right)^{2} \\
\alpha_{k} & =\left\|\mathbf{a}\left(r_{k}\right)^{H} \mathbf{U}_{\bullet} \Sigma_{\bullet}^{-1} \mathbf{V}_{\bullet}\right\|^{2} \\
\beta_{k} & =\left\|\mathbf{a}^{(1)}\left(r_{k}\right)^{H} U_{0}\right\|^{2}
\end{aligned}
$$




$$
\gamma_{k}=\frac{\|\mathbf{h}\|^{2}}{L-P} \prod_{\substack{j \neq k \\ j=1}}^{L-1}\left|\left(1-r_{j} r_{k}^{-1}\right)\right|^{2} .
$$

- State-Space Realization (SSR)

$$
\begin{aligned}
C & =\left(\frac{\lambda_{c}}{2 \pi d \cos \theta_{k}}\right)^{2} \\
\alpha_{k} & =\left\|\mathbf{v}_{k} \Sigma_{o}^{-\frac{1}{2}} \mathbf{U}_{\bullet}^{\dagger}\left(\mathbf{U}_{0}^{\dagger}-\lambda_{k} \mathbf{U}_{o}^{\dagger}\right) \mathbf{U}_{o}^{H}\right\|^{2} \\
\beta_{k} & =\left\|\mathbf{V}, \Sigma^{-\frac{1}{2}} \mathbf{u}_{k}\right\|^{2} \\
\gamma_{k} & =1 .
\end{aligned}
$$

- $\underline{\text { ESPRIT }}(\mathrm{EPR})$

$$
\begin{aligned}
C & =\left(\frac{\lambda_{e}}{2 \pi d \cos \theta_{k}}\right)^{2} \\
\alpha_{k} & =\left\|\mathbf{v}_{k}\left(\mathbf{U}_{o z}^{H} \mathrm{U}_{o z}\right)^{-1} \mathbf{U}_{o x}^{H}\left(\mathrm{U}_{o z}-\lambda_{k} \mathrm{U}_{o z}\right) \mathbf{U}_{o}^{H}\right\|^{2} \\
\beta_{k} & =\left\|\mathbf{V}_{\bullet} \Sigma_{\bullet}^{-1} \mathbf{u}_{k}\right\|^{2} \\
\gamma_{k} & =1 .
\end{aligned}
$$

The above results are listed in table 7.1.

\subsection{Comparative Examples}

The configuration of the experiment is: a twenty-element uniform line array (with $d=\lambda_{c} / 2$ ) with two sources at 0.2 and 0.5 radians (angles are measured with respect to the normal of the array). Twenty snapshots of array data were taken under $20 \mathrm{~dB}$ $\operatorname{SNR}\left[=10 \log \left(\frac{1}{\sigma_{n}^{2}}\right)\right]$ where the $\sigma_{n}^{2}$ is the variance of the complex, additive noise] for 500 hundred trials. The data matrices as in (2.2) were formed (for the purpose of comparison, we use forward-only formulation (F). Our other results show that the forward-back formulation decreases the mean-squared error of the Min-Norm 


$$
E\left(\Delta \theta_{k}^{2}\right)=\frac{C\left\|\alpha_{k}\right\|^{2}\left\|\beta_{k}\right\|^{2} \sigma_{n}^{2}}{2 \gamma_{k}^{2}}
$$

\begin{tabular}{|c|c|c|c|c|}
\hline & $C$ & $\left\|\alpha_{k}\right\|^{2}$ & $\left\|\beta_{k}\right\|^{2}$ & $\boldsymbol{\gamma}_{k}$ \\
\hline SMS & 1 & $\left\|\mathbf{a}\left(\theta_{k}\right)^{H} \mathrm{U}_{s} \mathbf{\Sigma}_{s}^{-1} \mathrm{~V}_{s}\right\|^{2}$ & $\left\|\mathbf{a}^{(1)}\left(\theta_{k}\right)^{H} U_{0}\right\|^{2}$ & $\left\|\mathbf{a}^{(1)}\left(\theta_{k}\right)^{H} \mathbf{U}_{o}\right\|^{2}$ \\
\hline SMN & 1 & $\left\|\mathrm{a}\left(\theta_{k}\right)^{H} U_{s} \Sigma_{e}^{-1} V_{s}\right\|^{2}$ & $\left\|\mathrm{a}^{(1)}\left(\theta_{k}\right)^{H} \mathrm{U}_{o}\right\|^{2}\|\mathrm{c}\|^{2}$ & $\left\|\mathrm{a}^{(1)}\left(\theta_{k}\right)^{H} \mathrm{U}_{o} \mathrm{c}\right\|^{2}$ \\
\hline RMN & $\left(\frac{\lambda_{c}}{2 \pi d \cos \sigma_{k}}\right)^{2}$ & $\left\|\mathrm{a}\left(r_{k}^{-1}\right)^{T} U_{s} \Sigma_{a}^{-1} V_{\bullet}\right\|^{2}$ & $\left\|a^{(1)}\left(r_{k}^{-1}\right)^{T} U_{0}\right\|^{2}\|c\|^{2}$ & $\prod_{\substack{j \neq k \\
j=1}}^{L-1}\left|\left(1-r_{j} r_{k}^{-1}\right)\right|^{2}$ \\
\hline RMS & $\left(\frac{\lambda_{c}}{2 \pi d \cos \sigma_{k}}\right)^{2}$ & $\left\|\mathrm{a}\left(r_{k}^{-1}\right)^{T} U_{,} \Sigma_{0}^{-1} V_{\bullet}\right\|^{2}$ & $\left\|\mathbf{a}^{(1)}\left(r_{k}^{-1}\right)^{T} U_{0}\right\|^{2}$ & 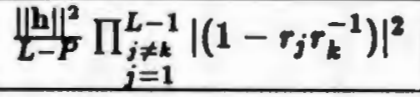 \\
\hline SSR & $\left(\frac{\lambda_{c}}{2 \pi d \cos \theta_{k}}\right)^{2}$ & $\left\|\mathbf{v}_{k} \Sigma_{s}^{-\frac{1}{2}} U_{s}^{!^{\dagger}}\left(\mathbf{U}_{o}^{\dagger}-\lambda_{k} \mathbf{U}_{o}^{!}\right) \mathbf{U}_{o}^{H}\right\|^{2}$ & $\left\|\mathbf{V}, \boldsymbol{\Sigma}^{-\frac{1}{2}} \mathbf{u}_{k}\right\|^{2}$ & 1 \\
\hline ESP & $\left(\frac{\lambda_{c}}{2 \pi d \cos \theta_{k}}\right)^{2}$ & $\left\|\mathrm{v}_{k}\left(\mathrm{U}_{s z}^{H} \mathrm{U}_{a z}\right)^{-1} \mathrm{U}_{g z}^{H}\left(\mathrm{U}_{o z}-\lambda_{k} \mathrm{U}_{o z}\right) \mathrm{U}_{o}^{H}\right\|^{2}$ & $\left\|\mathbf{V}, \boldsymbol{\Sigma}_{e}^{-1} \mathbf{u}_{k}\right\|^{2}$ & 1 \\
\hline
\end{tabular}

Table 7.1: Parameters of mean-squared estimation error 
estimation by a factor of 2$)$. The signals are $s_{k}(n)=e^{j\left(\frac{3 x_{0}}{x_{0}} n+\phi_{k n}\right)}$, where $\phi_{k n}$ are independent random phase angles uniformly distributed in the interval $(-\pi, \pi)$.

In the figures displayed here, the lines are theoretical prediction, and discrete symbols are simulation measurements. Solid and dashed lines (discrete symbols * and +) of MUSIC and Min-Norm are respectively for extrema searching and polynomial rooting approaches. We also use solid and dashed lines (also ${ }^{*}$ and + ) for State-Space Realization and ESPRIT. Statistical performances for all algorithms versus signal-to-noise ratio, source separation, number of sensors and number of snapshots are shown in Figures 7.1-4.

Under high SNR, all the algorithms appear to be unbiased in DOA estimation, so the important statistical characteristic is the mean-squared error of the estimation. With mean-squared error as the performance measure the subspace based algorithms get ranked as MUSIC, State-Space Realization and ESPRIT, Min-Norm (for the purpose of comparison, we used forward-only data). However, as indicated in a preliminary study of threshold (low SNR) performance, that at low SNR, the estimates' bias, rather than mean-squared error, assumes more importance in determining resolution. In this this case, with the bias as performance measure the algorithms can be ranked in the order of Min-Norm, ESPRIT and State-Space Realization, MUSIC. 


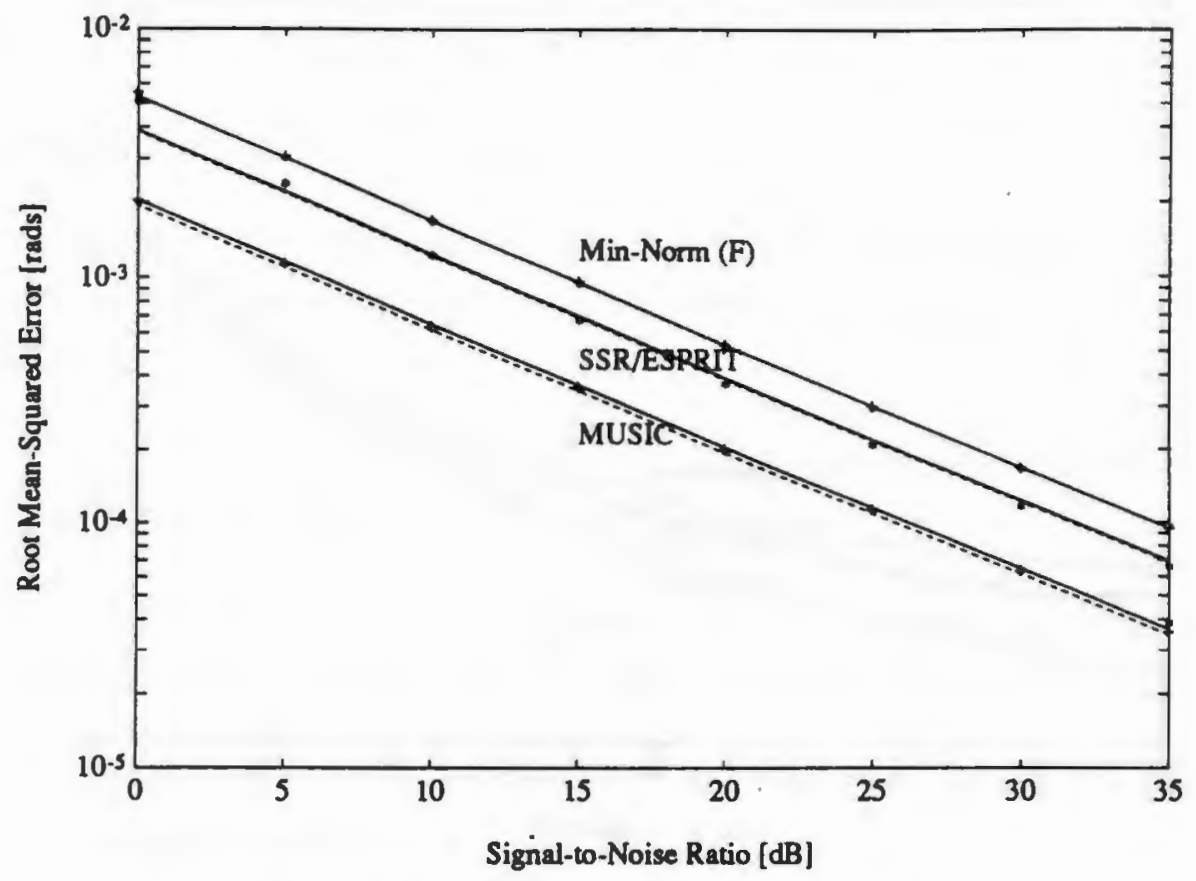

Figure 7.1: RMSE vs. Signal-to-Noise Ratio

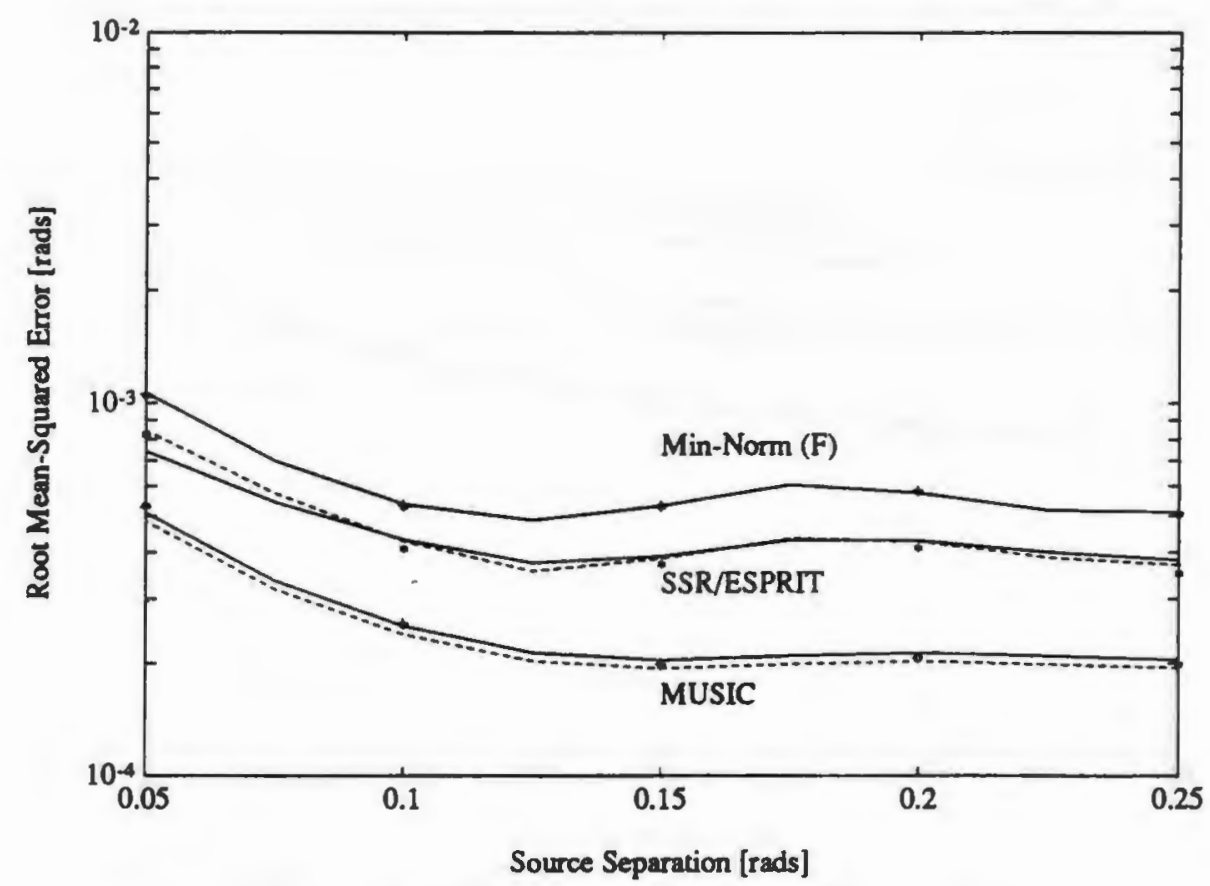

Figure 7.2: RMSE vs. Source Separation 


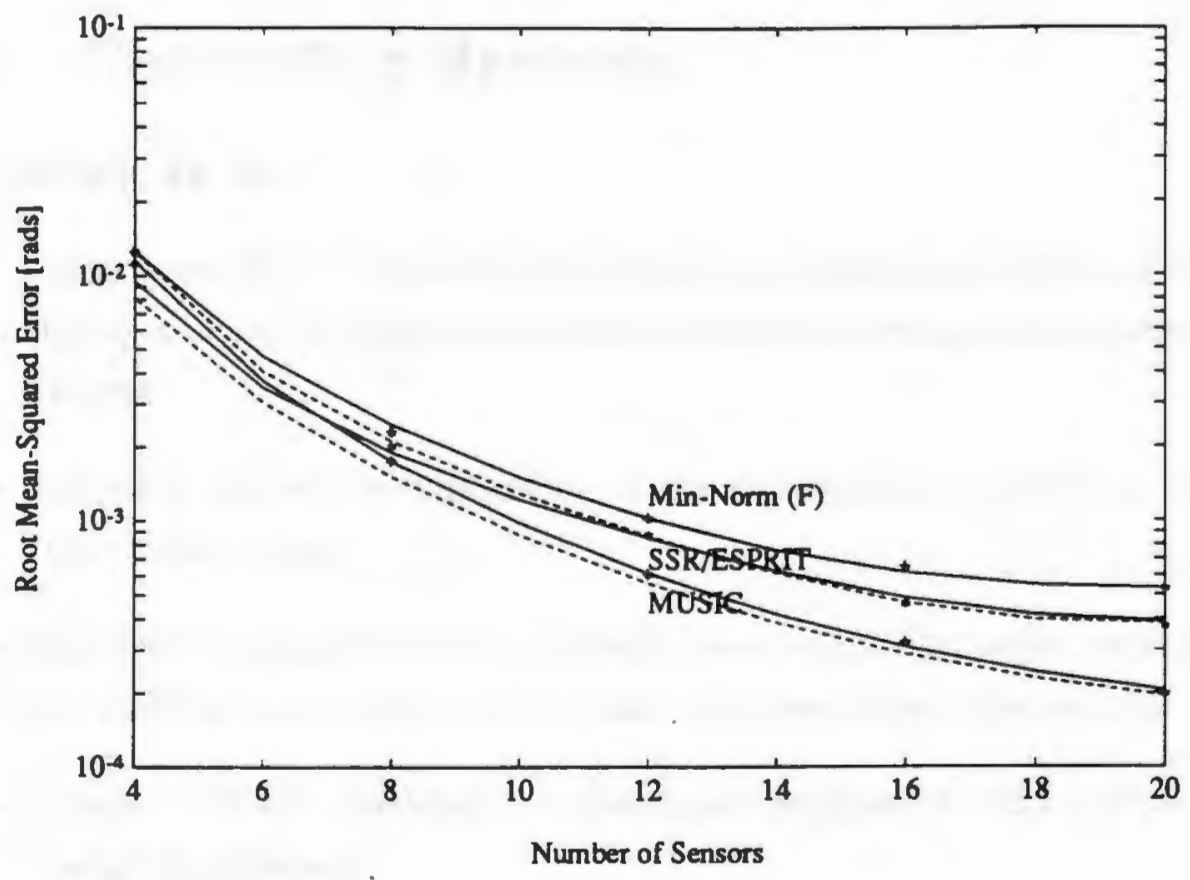

Figure 7.3: RMSE vs. Number of Sensors

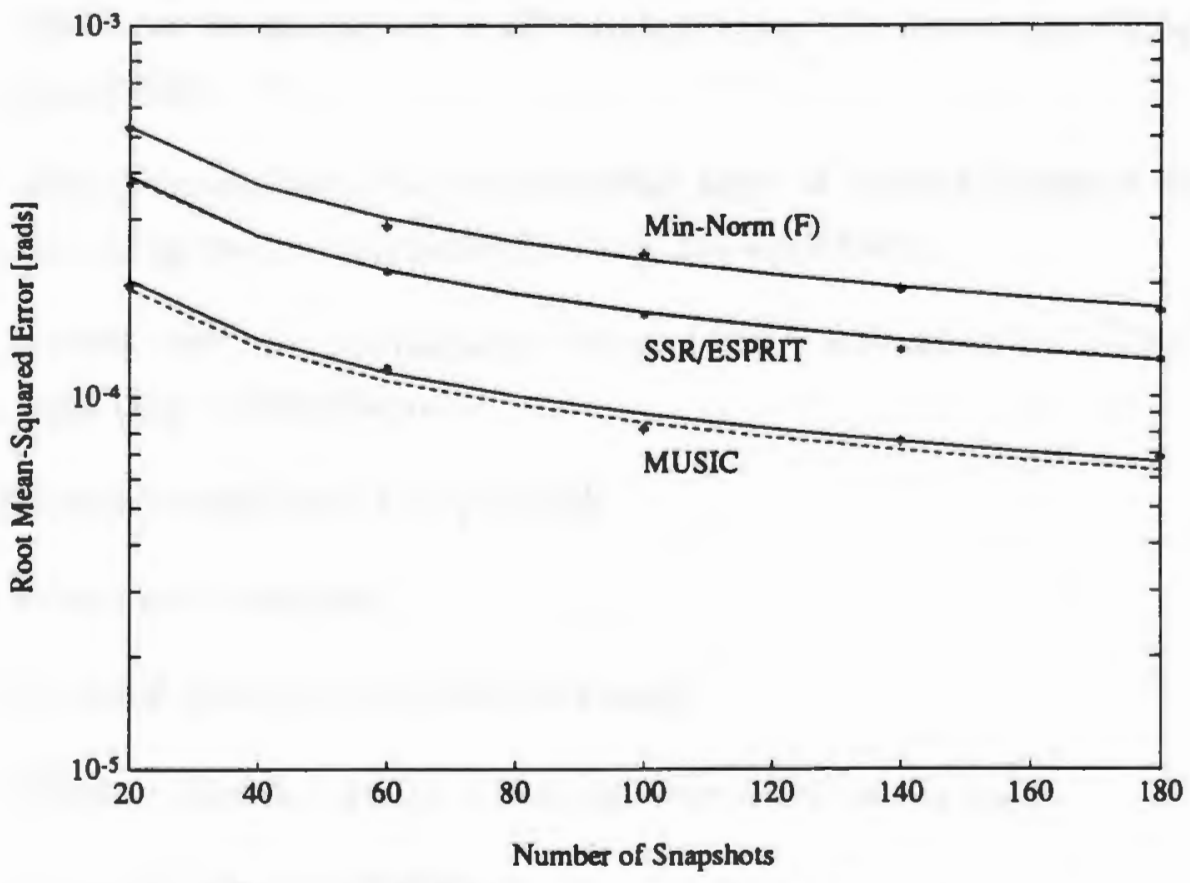

Figure 7.4: RMSE vs. Number of Snapshots 


\subsection{Concluding Remarks}

In summary, we have

- categorized DOA estimation algorithms into orthogonal subspace based algorithms and signal subspace based algorithms according to the subspace being utilized.

- derived a first-order expression of the subspace perturbations induced by observation noise.

- classified the algorithms into extrema searching, polynomial rooting and matrix shifting approaches by the numerical procedures they exploit.

- obtained a linear relationship between perturbation of DOA and perturbation of a given subspace.

- established a general expression of mean-squared error of DOA estimation which can be specialized to all the algorithms by substituting the appropriate parameters.

- demonstrated that the mean-squared error of DOA estimation by extrema searching and by polynomial rooting are equivalent.

- proved that the mean-squared error of DOA estimation by MUSIC is lower than that by Min-Norm.

The following areas should be pursued:

- sensor error analysis;

- low SNR analysis (threshold analysis);

- coherent interference (or multipath time-delay) analysis;

- wideband signal performance. 


\section{Appendix A}

\section{Mean-Squared Error Calculation}

Here we derive equation (4.16). We start by writing

$$
\begin{aligned}
& E\left[\Re\left(\alpha^{H} \mathrm{~N} \beta\right)\right]^{2} \\
= & E\left(\frac{\alpha^{H} \mathrm{~N} \beta+\beta^{H} \mathrm{~N}^{H} \alpha}{2}\right)^{2} \\
= & \frac{1}{4} E\left[\left(\alpha^{H} \mathrm{~N} \beta+\beta^{H} \mathrm{~N}^{H} \alpha\right)\left(\beta^{H} \mathrm{~N}^{H} \alpha+\alpha^{H} \mathrm{~N} \beta\right)\right] \\
= & \frac{1}{2} \alpha^{H} E\left(\mathrm{~N} \beta \beta^{H} \mathrm{~N}^{H}\right) \alpha+\frac{1}{4} \alpha^{H} E\left(\mathrm{~N} \beta \alpha^{H} \mathrm{~N}\right) \beta+\frac{1}{4} \beta^{H} E\left(\mathrm{~N}^{H} \alpha \beta^{H} \mathrm{~N}^{H}\right) \alpha \\
= & \frac{1}{2} E\left(\alpha^{H} \mathrm{~N} \beta\right)^{2}+\frac{1}{4} \alpha^{H} E\left(\mathrm{~N} \beta \alpha^{H} \mathrm{~N}\right) \beta+\frac{1}{4} \beta^{H} E\left(\mathbf{N}^{H} \alpha \beta^{H} \mathrm{~N}^{H}\right) \alpha .
\end{aligned}
$$

If the real and imaginary parts of the noise matrix $\mathbf{N}$ are independent Gaussian random variables with zero mean and variance of $\frac{1}{\sigma^{2}}$, then for each element $n$ of the noise matrix $\mathbf{N}$, we have

$$
\begin{aligned}
E(n n) & =E\left(n_{\circledast}+j n_{\Im}\right)\left(n_{\circledast}+j n_{\Im}\right) \\
& =\frac{1}{2} \sigma^{2}-\frac{1}{2} \sigma^{2} \\
& =0 .
\end{aligned}
$$

Now consider the second term of (A.1). We expand $\mathbf{N} \beta$ as a weighted sum of the columns of $N$, and expand $\alpha^{H} N$ as a weighted sum of the rows of $N$ and use (A.2) 
to obtain

$$
\begin{aligned}
E\left(\mathbf{N} \beta \alpha^{H} \mathbf{N}\right) & =E\left[\sum_{i} \beta(i) \underline{\mathbf{n}}_{i} \sum_{j} \alpha(j)^{*} \mathbf{n}_{j}\right] \\
& =\sum_{i} \sum_{j}\left[\beta(i) \alpha(j)^{*}\right] E\left(\underline{\mathbf{n}}_{i} \overline{\mathbf{n}}_{j}\right) \\
& =0
\end{aligned}
$$

where $\underline{\underline{n}}_{i}$ and $\tilde{\mathbf{n}}_{j}$ denote the $i$-th column and $j$-th row of $\mathbf{N}$, and the $\beta(i)$ and $\alpha(j)^{*}$ are the $i$-th and $j$-th elements of the $\beta$ and $\alpha^{B}$ vectors, respectively. In a similar way, it can be shown that the third term of (A.1) is zero. This proves that

$$
\begin{aligned}
E\left[\Re\left(\alpha^{H} \mathrm{~N} \beta\right)\right]^{2} & =\frac{1}{2} \alpha^{H} E\left(\mathrm{~N} \beta \beta^{H} \mathrm{~N}^{H}\right) \alpha \\
& =\frac{1}{2} \dot{E}\left|\alpha^{H} \mathrm{~N} \beta\right|^{2} .
\end{aligned}
$$

Furthermore, since

$$
\begin{aligned}
E\left(n n^{*}\right) & =E\left(n_{\circledast}+j n_{\Im}\right)\left(n_{\circledast}-j n_{\Im}\right) \\
& =\frac{1}{2} \sigma^{2}+\frac{1}{2} \sigma^{2} \\
& =\sigma^{2},
\end{aligned}
$$

we can write

$$
\begin{aligned}
E\left(\mathbf{N} \beta \beta^{H} \mathbf{N}^{H}\right) & =E\left[\sum_{i} \beta(i) \underline{n}_{i} \sum_{j} \beta(j)^{*} \underline{\underline{n}}_{j}^{H}\right] \\
& =\sum_{i} \sum_{j}\left[\beta(i) \beta(j)^{*}\right] E\left(\underline{n}_{i} \underline{\underline{n}}_{j}^{H}\right) \\
& =\sum_{i} \sum_{j}\left[\beta(i) \beta(j)^{*}\right] \sigma^{2} \mathbf{I} \delta(i-j) \\
& =\|\beta\|^{2} \sigma^{2} \mathbf{I} .
\end{aligned}
$$

Using (A.4) and the above equation, we have

$$
E\left[\Re\left(\alpha^{H} \mathrm{~N} \beta\right)\right]^{2}=\frac{1}{2}\|\alpha\|^{2}\|\beta\|^{2} \sigma^{2} .
$$




\section{Appendix B}

\section{Pisarenko's Method}

The analysis presented in this research can be easily applied to Pisarenko's method [22]. Pisarenko's Method is the first subspace based estimation algorithm, originately designed for the harmonic retrieval problem. Pisarenko's Method applied principal component decomposition on a covariance matrix of dimension $(P+1) \times(P+1)]$. Then the $P+1$-th eigenvector $\mathbf{u}_{P+1}$ is in the orthogonalsubspace which is perpendicular to the signal manifold at a source direction. The covariance matrix, limited to be dimension of $[(P+1) \times(P+1)]$, can be estimated from array data in a spatial smoothing manner of shifted subarray data [73]. Pisarenko's method was originally designed as a polynomial-rooting algorithm for uniform line array, but it can be easily applied to extrema-searching algorithm for the array of arbitrary geometry, like we did for the Min-Norm method.

The orthogonality relationship is

$$
\mathrm{a}\left(\theta_{k}\right) \mathrm{u}_{P+1}=0 \text { for } k=1, \ldots, P .
$$

For a uniform line array the above equation can viewed as a polynomial evaluated at the signal-roots with $z=e^{j \frac{2 \pi d}{\lambda_{e}} \sin \theta_{k}}$. The searching algorithm is to find these minima over $\theta$ where $\theta$ is in

$$
a_{i}(\theta)=e^{j \frac{2 \pi t}{\lambda_{\theta}}\left(x_{i} \sin \theta+y_{i} \cos \theta\right)} .
$$

The statistical performance can be analyzed in the similar procedure as in the analyses for MUSIC and Min-Norm. 
Polynomial-Rooting algorithm:

The bias of estimated direction of arrival is

$$
\begin{aligned}
E\left(\Delta \theta_{k}\right) & =C_{k} \frac{\Im\left[\mathbf{a}\left(r_{k}^{-1}\right)^{T} \mathbf{U}_{,} \Sigma_{a}^{-1} \mathbf{V}_{,}^{H} E\left(\mathbf{N}^{H}\right) \mathbf{u}_{P+1} \mathbf{u}_{P+1}^{H} \mathbf{a}^{(1)}\left(r_{k}\right)\right]}{A r_{k}^{*} G\left(r_{k}\right)} \\
& =0 .
\end{aligned}
$$

The mean-squared error of DOA estimation is

$$
\begin{aligned}
E\left(\Delta \theta_{k}\right)^{2} & =\frac{C_{k}^{2}}{2} \frac{\left(\alpha_{k}^{H} \alpha_{k}\right)\left(\beta_{k}^{H} \beta_{k}\right) \sigma^{2}}{A^{2}\left|G\left(r_{k}\right)\right|^{2}} \\
& =\frac{C_{k}^{2}}{2} \frac{\left\|\alpha_{k}\right\|^{2} \sigma^{2}}{u_{P+1}^{2}(1) G\left(r_{k}\right)}
\end{aligned}
$$

where

$$
\alpha_{k}=\mathbf{V}, \boldsymbol{\Sigma}_{s}^{-1} \mathbf{U}_{s}^{H} \mathbf{a}\left(r_{k}\right)
$$

and

$$
\beta_{k}=\mathbf{u}_{P+1} \mathbf{u}_{P+1}^{H} \mathbf{a}^{(1)}\left(r_{k}\right)
$$

and

$$
A=\mathrm{u}_{P+1}^{2}(1)
$$

$\mathbf{u}_{P+1}(1)$ is the first entry of vector $\mathbf{u}_{P+1}$.

Extrema-Searching algorithm:

The bias of the estimated direction of arrival is

$$
\begin{aligned}
E\left(\Delta \theta_{k}\right) & =\frac{\Re\left[-\mathbf{a}\left(\theta_{k}\right)^{H} \mathbf{U}_{s} \Sigma_{g}^{-1} \mathbf{V}_{g}^{H} E\left(\mathbf{N}^{H}\right) \mathbf{u}_{P+1} \mathbf{u}_{P+1}^{H} \mathbf{a}^{(1)}\left(\theta_{k}\right)\right]}{\mathbf{a}^{(1)}\left(\theta_{k}\right)^{H} \mathbf{u}_{P+1} \mathbf{u}_{P+1}^{H} \mathbf{a}^{(1)}\left(\theta_{k}\right)} \\
& =0 .
\end{aligned}
$$

The mean-squared error of DOA estimation is

$$
\begin{aligned}
E\left(\Delta \theta_{k}\right)^{2} & =\frac{\left(\alpha_{k}^{H} \alpha_{k}\right)\left(\beta_{k}^{H} \beta_{k}\right) \sigma_{n}^{2}}{2\left[\mathbf{a}^{(1)}\left(\theta_{k}\right)^{H} \mathbf{u}_{P+1} \mathbf{u}_{P+1}^{H} \mathbf{a}^{(1)}\left(\theta_{k}\right)\right]^{2}} \\
& =\frac{\left\|\alpha_{k}\right\|^{2} \sigma_{n}^{2}}{2\left\|\mathbf{a}^{(1)}\left(\theta_{k}\right)^{H} \mathbf{u}_{P+1}\right\|^{2}}
\end{aligned}
$$


Appendix $B$.

where

$$
\alpha_{k}=\mathrm{V}_{\imath} \Sigma_{\bullet}^{-1} \mathrm{U}^{H} \mathrm{a}\left(\theta_{k}\right)
$$

and

$$
\beta_{k}=\mathbf{u}_{P+1} \mathbf{u}_{P+1}^{H} \mathbf{a}^{(1)}\left(\theta_{k}\right) .
$$




\section{Appendix C}

\section{Perturbation of Angle and Root}

The relation between an arrival angle and a signal root of the characteristic polynomial (or eigenvalue of the signal transition matrix) can be derived as follows:

The noise-free signal roots are

$$
r=e^{j \frac{2 r \Delta}{\alpha} \sin \theta}
$$

and the signal roots in noisy case are

$$
\tilde{r}=\tilde{c} e^{j \frac{2 \pi \Delta}{d} \sin \bar{\theta} .}
$$

where $\tilde{r}=r+\Delta r$. Define an intermediate variable $\omega$

$$
\omega=\frac{2 \pi \Delta}{\lambda} \sin \theta
$$

with

$$
\tilde{\omega} \approx \frac{2 \pi \Delta}{\lambda} \sin \tilde{\theta}
$$

and $\tilde{\omega}=\omega+\Delta \omega$. It is easy to show that

$$
\Delta \omega=\frac{2 \pi \Delta}{\lambda} \cos \theta \Delta \theta .
$$

Define

$$
f(r)=\ln (r),
$$


and expand $\ln (\tilde{r})$ into a power series terms, and keep only the first two terms:

$$
f(\tilde{r}) \approx f(r)+f^{\prime}(r) \Delta r
$$

Using (C.1), we can write

$$
\ln \tilde{c}+j \Delta \omega=f^{\prime}(r) \Delta r
$$

where $f^{\prime}(r)=\frac{1}{r}$. The imaginary part in the both sides of the equation must be equal

$$
\Delta \omega=\Im\left[\frac{\Delta r}{r}\right]
$$

Using (C.2), we obtain

$$
\Delta \theta=C \Delta \omega=C \Im\left[\frac{\Delta r}{r}\right]
$$

where $C$ is defined as

$$
C \stackrel{\text { def }}{=} \frac{\lambda}{2 \pi \Delta \cos \theta}
$$




\section{Appendix D}

\section{Cramer-Rao Bound Calculation}

The formula for calculating the Cramer-Rao Lower Bound used in this research is suggested by Clergeot, Tressens and Ouamri. The interested reader can find the detailed derivations in [5]. For uncorrelated signals, the lower bound of meansquared error can be expressed as

$$
E\left[\left(\tilde{\theta}_{k}-\theta_{k}\right)^{2}\right] \leq \frac{\sigma_{n}^{2}}{2 M p_{k}\left[\mathbf{a}^{(1)}\left(\theta_{k}\right)^{B} \Pi_{o} a^{(1)}\left(\theta_{k}\right)\right]}
$$

where $p_{k}$ is the power of $k$-th signal calculated from $k$-th diagonal element of $\mathbf{R}_{s}=E\left(\mathbf{s s}^{H}\right) . \Pi_{0}$ is the projector on orthogonal-subspace, defined as

$$
\mathbf{\Pi}_{0}=\mathbf{U}_{0}\left(\mathbf{U}_{0}^{H} \mathrm{U}_{0}\right)^{-1} \mathrm{U}_{0}^{H}
$$

where $\mathrm{U}_{0}$ are the left-singular vectors associated with $L-P$ smallest singular values from the noise-free data matrix. The approach we used, independent from data, is

$$
\begin{aligned}
\Pi_{0} & =\mathbf{I}-\mathrm{U}_{0}\left(\mathrm{U}_{\bullet}^{H} \mathrm{U}_{0}\right)^{-1} \mathbf{U}^{H} \\
& =\mathbf{I}-\sum_{k=1}^{P}\left\{\mathbf{a}\left(\theta_{k}\right)\left[\mathbf{a}\left(\theta_{k}\right)^{H} \mathbf{a}\left(\theta_{k}\right)\right]^{-1} \mathbf{a}\left(\theta_{k}\right)^{H}\right\} .
\end{aligned}
$$




\section{References}

[1] A. J. Barabell, "Improving the resolution performance of eigenstructure-based direction-finding algorithm," in Proc. IEEE ICASSP'89, pp. 336-339, 1983.

[2] M. Kaveh and A. J. Barabell, "The statistical performance of the MUSIC and the Minimum-Norm algorithms in resolving plane-waves in noise," IEEE Trans. Acoustics, Speech, and Signal Processing, vol. ASSP-34, pp. 331-340, April 1986.

[3] B. Porat and B. Friedlander, "An accuracy analysis of the Kumaresan-Tufts method for estimating complex damped exponentials," IEEE Trans. Acoustics, Speech, and Signal Processing, vol. ASSP-35, pp. 231-235, February 1987.

[4] B. Porat and B. Friedlander, "Analysis of the asymptotic relative efficiency of the MUSIC algorithm," IEEE Trans. Acoustics, Speech, and Signal Processing, vol. ASSP-36, pp. 532-543, April 1988.

[5] H. Clergeot, S. Tressens, and A. Ouamri, "Performance of high resolution frequencies estimation methods compared to the Cramer-Rao bounds," IEEE Trans. Acoustics, Speech, and Signal Processing, vol. ASSP-37, pp. 1703-1720, November 1989.

[6] Y. Hua and T. K. Sarkar, "Perturbation analysis of TK method for harmonic retrieval problems," IEEE Trans. Acoustics, Speech, and Signal Processing, vol. ASSP-36, pp. 228-240, February 1988. 
[7] D. V. Bhaskar Rao, "Perturbation analysis of an SVD-based Linear Prediction method for estimating the frequencies of multiple sinusoids," IEEE Trans. Acoustics, Speech, and Signal Processing, vol. ASSP-36, pp. 1026-1035, July 1988.

[8] D. V. Bhaskar Rao and K. V. S. Hari, "Statistical performance analysis of Minimum-Norm method," IEE Proc., vol. 136, Pt.F, pp. 125-134, June 1989.

[9] D. V. Bhaskar Rao and K. V. S. Hari, "Performance analysis of Root-MUSIC," IEEE Trans. Acoustics, Speech, and Signal Processing, vol. ASSP-37, pp. 1939-1949, December 1989.

[10] D. V. Bhaskar Rao, "Sensitivity consideration in state-space model-based harmonic retrieval methods," IEEE Trans. Acoustics, Speech, and Signal Processing, vol. ASSP-37, pp. 1789-1793, November 1989.

[11] P. Stoica and A. Nehorai, "MUSIC, Maximum Likelihood and Cramer-Rao bound," in Proc. IEEE ICASSP'88, (New York, NY), pp. 2296-2299, April 1988.

[12] P. Stoica and A. Nehorai, "MUSIC, Maximum Likelihood and Cramer-Rao bound: Further results and comparisons," in Proc. IEEE ICASSP'89, (Glasgow, UK), pp. 2605-2608, April 1989.

[13] P. Stoica and A. Nehorai, "MUSIC, Maximum Likelihood and Cramer-Rao bound," IEEE Trans. Acoustics, Speech, and Signal Processing, vol. ASSP-37, pp. 720-741, May 1989.

[14] D. R. Farrier, D. J. Jeffries, and R. Mardani, "Perturbation analysis of the MUSIC algorithm," in Proc. IEEE ICASSP'88, (New York, NY), pp. 28732876, April 1988.

[15] D. R. Farrier, D. J. Jeffries, and R. Mardani, "Theoretical performance prediction of the MUSIC algorithm," IEE Proc., Pt. F, vol. 135, pp. 216-225, June 1988. 
[16] D. W. Tufts, R. J. Vaccaro, and A. C. Kot, “Analysis of estimation of signal parameters by linear prediction at high SNR using matrix approximation," in Proc. IEEE ICASSP'89, (Glasgow, UK), May 1989.

[17] F. Li, R. J. Vaccaro, and D. W. Tufts, "Min-Norm Linear Prediction for arbitrary sensor array," in Proc. IEEE ICASSP'89, (Glasgow, UK), pp. 26132616, May 1989.

[18] R. Prony, "Essai experimental et analytique etc.," L'ecole Polytechnique, pp. 24-76, 1795.

[19] J. W. Cooley and J. W. Turkey, "An algorithm for the machine calculation of fourier series," Math. Comput, vol. 19, pp. 297-301, 1965.

[20] J. P. Burg, "Maximum entropy spectral analysis," in Proc. 97th Meeting of the Society of Exploration Geophysicists, pp. 34-41, 1967.

[21] J. Capon, "High-resolution frequency-wavenumber spectrum analysis," Proc. IEEE, vol. 57, pp. 1408-1418, 1969.

[22] V. F. Pisarenko, "The retrieval of harmonics from a covariance function," Geophys. J. Royal Astronom. Soc., vol. 33, pp. 347-366, 1976.

[23] R. O. Schmidt, "Multiple emitter location and signal parameter estimation," in Proc. RADC Spectral Estimation Workshop, (Griffiss AFB, NY), pp. 243$258,1979$.

[24] R. O. Schmidt, A Signal Subspace Approach to Multiple Emitter Location and Spectral Estimation. PhD thesis, Stanford University, Stanford, CA, 1981.

[25] G. Bienvenu and L. Kopp, "Principle de la Goniometric passive adaptive," in Proc. 7'eme Colloque GRESIT, (Nic, France), pp. 106/1-106/10, 1979.

[26] D. Johnson, "The application of spectral estimation methods to bearing estimation problem," Proc. IEEE, vol. 70, pp. 1018-1028, 1982. 
[27] D. W. Tufts and R. Kumaresan, "Estimation of frequencies of multiple sinusoids: Making linear prediction perform like maximum likelihood," Proc. IEEE, vol. 70, pp. 975-989, September 1982.

[28] R. Kumaresan and D. W. Tufts, "Estimating the angles of arrival of multiple plane waves," IEEE Trans. Aerosp., Electron. Syst., vol. AES-19, pp. 134-139, January 1983.

[29] S. Y. Kung, D. V. Bhaskar Rao, and K. S. Arun, "New state space and singular value decomposition based approximate modeling methods for harmonic retrieval," in IEEE 2nd ASSP Workshop on Spectral Estimation \& Modeling, pp. 266-271, August 1983.

[30] S. Y. Kung, K. S. Arun, and D. V. Bhaskar Rao, "State-space and singularvalue decomposition-based approximation methods for the harmonic retrieval problem," J. Opt. Soc. Am., vol. 73, pp. 1799-1811, December 1983.

[31] R. Foka and S. Y. Kung, "A novel optimum energy solution in iterative constrained restoration," in Proc. EUSIPCO'88, pp. 1041-1044, Science Publishers B. V., 1986.

[32] R. Foka, "Comparison of high resolution spectral methods based on SVD," in Proc. IEEE ICASSP'87, (Dallas, TX), pp. 2233-2236, April 1987.

[33] A. Paulaj, R. Roy, and T. Kailath, "Estimation of signal parameters via rotational invariance techniques - ESPRIT," in Proc. $19^{\text {th }}$ Asilomar Conf. on Signals, Systems and Computers, (Pacific Grove, CA), pp. 83-89, November 1985.

[34] R. Roy, A. Paulaj, and T. Kailath, "Direction-of-arrival estimation by subspace rotation methods - ESPRIT," in Proc. IEEE ICASSP'86, (Tokyo, Japan), pp. 47.2.1-47.2.4, April 1986.

[35] A. Paulaj, R. Roy, and T. Kailath, "A subspace rotation approach to parameter estimation," Proc. IEEE, vol. 70, pp. 1044-1045, July 1986. 
[36] R. Roy, A. Paulaj, and T. Kailath, "Estimation of signal parameters via rotational invariance techniques - ESPRIT," in Proc. IEEE MILCON, pp. 41.6.141.6.5, 1986.

[37] R. Roy, A. Paulaj, and T. Kailath, "Comparative performance analysis of ESPRIT and MUSIC direction-of-arrival estimation," in Proc. $20^{\text {th }}$ Asilomar Conf. on Signals, Systems and Computers, (Pacific Grove, CA), pp. 580-584, November 1986.

[38] R. Roy, A. Paulaj, and T. Kailath, "Comparative performance analysis of ESPRIT and MUSIC direction-of-arrival estimation," in Proc. IEEE ICASSP'87, (Dallas, TX), pp. 2344-2347, April 1987.

[39] R. Roy, ESPRIT - Estimation of Signal Parameters Via Rotational Invariance Techniques. PhD thesis, Stanford University, Stanford, CA, 1987.

[40] R. Roy and T. Kailath, "Total least-squares ESPRIT," in Proc. 21" Asilomar Conf. on Signals, Systems and Computers, (Pacific Grove, CA), pp. 580-584, November 1987.

[41] R. Roy and T. Kailath, "ESPRIT - estimation of signal parameters via rotational invariance techniques," IEEE Trans. Acoustics, Speech, and Signal Processing, vol. ASSP-37, pp. 984-995, July 1989.

[42] S. Mayrargue, "ESPRIT and TAM (toeplitz approximation method) are theoretically equivalent," in IEEE Proc. ICASSP'88, (New York, NY), pp. 24562459, April 1988.

[43] S. Mayrargue and J. P. Jouveau, "On equivalence between esprit and tam (toeplitz approximation method), lead to an improved tam," in Proc. EUSIPCO'88, pp. 55-58, Science Publishers B. V., 1988.

[44] H. Ouibrahim, D. D. Weiner, and T. K. Sarkar, "A general approach to direction-finding," in Proc. IEEE MILCON, pp. 41.4.1-41.4.5, 1986. 
[45] H. Ouibrahim, D. D. Weiner, and T. K. Sarkar, "Matrix pencil approach to angle of arrival estimation," in Proc. $20^{\text {th }}$ Asilomar Conf. on Signals, Systems and Computers, (Pacific Grove, CA), pp. 203-206, November 1986.

[46] H. Ouibrahim, D. D. Weiner, and T. K. Sarkar, "A general approach to direction-finding," IEEE Trans. Acoustics, Speech, and Signal Processing, vol. ASSP-36, pp. 610-612, April 1988.

[47] Y. Hua and T. K. Sarkar, "Matrix pencil method and its performance," in Proc. IEEE ICASSP'88, (New York, NY), pp. 2476-2479, April 1988.

[48] K. Sharman and T. S. Durrani, "A comparative study of modern eigenstructure methods for bearing estimation - a new high performance approach," in Proc. 25 ${ }^{\text {th }}$ IEEE Conf., on Decision and Control, pp. 1737-1742, 1986.

[49] M. D. Zoltowski, "Novel techniques for estimation of array signal parameters based on matrix pencil, subspace rotations, and total least squares," in Proc. IEEE ICASSP'88, (New York, NY), pp. 2861-2864, April 1988.

[50] D. V. Bhaskar Rao and K. V.S. Hari, "Performance analysis of subspace based methods, in IEEE fth ASSP Workshop on Spectral Estimation 8 Modeling, (Minnesota, MN), pp. 92-97, August 1988.

[51] F. Li and R. J. Vaccaro, "MUSIC performance prediction by matrix approximation at high SNR," in Proc. 1989 Conf. Information Sciences and Systems, (The Johns Hopkins University, Baltimore, MD), pp. 447-481, March 1989.

[52] F. Li, R. J. Vaccaro, and D. W. Tufts, "Unified performance analysis of subspace-based estimation algorithms," in Proc. IEEE ICASSP'go, (Albuquerque, NM), pp. 2575-2578, April 1990.

[53] A. C. Kot, Perturbation Analysis of SVD-Based Methods. PhD thesis, University of Rhode Island, Kingston, RI, 1989.

[54] G. W. Stewart, "Stochastic perturbation theory," in UMIACS-TR-SS-76, (College Park, MD), pp. 1-24, October 1988. 
[55] G. W. Stewart, "Perturbation theory for the singular value decomposition," in Proc. 2nd International Workshop on SVD and Signal Processing, (Kingston, RI), June 1990.

[56] R. J. Vaccaro, D. W. Tufts, and G. F. Boudreaux-Bartels, $S V D$ and Signal Processing, ch. Advances in Principal Component Signal Processing, pp. 115148. E.F. Deprettere, ed., North-Holland, 1988.

[57] D. Spielman, A. Paulraj, and T. Kailath, "Performance analysis of the MUSIC algorithm," in Proc. IEEE ICASSP'86, (Tokyo, Japan), pp. 1909-1912, April 1986.

[58] B. Porat and B. Friedlander, "On the asymptotic relative efficiency of the MUSIC algorithm," in Proc. IEEE ICASSP'88, (New York, NY), pp. 23762379, April 1988.

[59] B. Friedlander, "A sensitivity analysis of the MUSIC algorithm," in Proc. IEEE ICASSP'89, (Glasgow, UK), pp. 2811-2814, May 1989.

[60] D. V. Bhaskar Rao and K. V. S. Hari, "Statistical performance analysis of Minimum-Norm method," in Proc. IEEE ICASSP'89, (Glasgow, UK), pp. 2760-2763, May 1989.

[61] F. Li and R. J. Vaccaro, "Analysis of music and min-norm for arbitrary array geometry," IEEE Trans. Aerosp., Electron. Syst., vol. AES-27, January 1991.

[62] A. C. Kot, S. Parthasarathy, D. W. Tufts, and R. J. Vaccaro, "Statistical performance of single sinusoid frequency estimation in white noise using statevariable balancing and Linear Prediction," IEEE Trans. Acoustics, Speech, and Signal Processing, vol. ASSP-35, pp. 1639-1642, November 1987.

[63] F. Li and R. J. Vaccaro, "A unified performance analysis of subspace-based polynomial-rooting algorithms for doa estimation," in Proc. 1990 Conf. Information Sciences and Systems, (Princeton, NJ), March 1990. 
[64] D. V. Bhaskar Rao, "Perturbation of a SVD based method for the harmonic retrieval problem," in Proc. IEEE ICASSP 85, pp. 624-627, April 1985.

[65] D. V. Bhaskar Rao, "Sensitivity analysis of state-space methods in spectrum estimation," in Proc. IEEE ICASSP'87, (Dallas, TX), pp. 1517-1520, April 1987.

[66] J. Le Cadre and P. Ravazzola, "Approximated stochastic realization and model reduction methods applied to array processing by means of state space models," in Proc. IEEE ICASSP'89, (Glasgow, UK), pp. 2601-2604, May 1989.

[67] L. B. Jackson, Digital Filters and Signal Processing. Boston, MA: Kluwer Academic Publishers, 1986.

[68] K. S. Arun, A Principal Components Approach to Approximate Modeling And ARMA Spectral Estimation. PhD thesis, University of Southern California, Los Angeles, CA, 1983.

[69] D. V. Bhaskar Rao and K. V. S. Hari, "Quality of low rank approximants obtained using SVD and some applications," in Proc. $21^{\text {st }}$ Annual Asilomar Conf. on Signals, Systems and Computers, (Pacific Grove, CA), pp. 784-787, November 1987.

[70] D. V. Bhaskar Rao, "Lowering the threshold of singular value decomposition based methods," in Proc. IEEE ICASSP'88, (New York, NY), pp. 2472-2475, April 1988.

[71] D. V. Bhaskar Rao and K. V. S. Hari, "Performance analysis of ESPRIT and TAM in determining the direction of arrival of plane waves in noise, IEEE Trans. Acoustics, Speech, and Signal Processing, vol. ASSP-37, pp. 1990-1995, December 1989.

[72] P. Lancaster and M. Tismentsky, The Theory of Matrices. New York, NY: Academic Press, second ed., 1978. 
[73] T. J. Shan, M. Wax, and T. Kailath, "On spatial smoothing for direction-ofarrival estimation for coherent signals," IEEE Trans. Acoustics, Speech, and Signal Processing, vol. ASSP-33, pp. 806-811, August 1985. 


\section{Bibliography}

Arun, K. S., A Principal Components Approach to Approximate Modeling And ARMA Spectral Estimation. PhD thesis, University of Southern California, Los Angeles, CA, 1983.

Barabell, A. J., "Improving the resolution performance of eigenstructure-based direction-finding algorithm," in Proc. IEEE ICASSP'89, pp. 336-339, 1983.

Bhaskar Rao, D. V., "Lowering the threshold of singular value decomposition based methods," in Proc. IEEE ICASSP'88, (New York, NY), pp. 2472-2475, April 1988.

Bhaskar Rao, D. V., "Perturbation analysis of an SVD-based Linear Prediction method for estimating the frequencies of multiple sinusoids," IEEE Trans. Acoustics, Speech, and Signal Processing, vol. ASSP-36, pp. 1026-1035, July 1988.

Bhaskar Rao, D. V., "Perturbation of a SVD based method for the harmonic retrieval problem," in Proc. IEEE ICASSP 85, pp. 624-627, April 1985.

Bhaskar Rao, D. V., "Sensitivity analysis of state-space methods in spectrum estimation," in Proc. IEEE ICASSP'87, (Dallas, TX), pp. 1517-1520, April 1987.

Bhaskar Rao, D. V., "Sensitivity consideration in state-space model-based harmonic retrieval methods," IEEE Trans. Acoustics, Speech, and Signal Processing, vol. ASSP-37, pp. 1789-1793, November 1989. 
Bhaskar Rao, D. V. and Hari, K. V. S., "Performance analysis of ESPRIT and TAM in determining the direction of arrival of plane waves in noise," IEEE Trans. Acoustics, Speech, and Signal Processing, vol. ASSP-37, pp. 1990-1995, December 1989.

Bhaskar Rao, D. V. and Hari, K. V. S., "Performance analysis of RootMUSIC," IEEE Trans. Acoustics, Speech, and Signal Processing, vol. ASSP-37, pp. 1939-1949, December 1989.

Bhaskar Rao, D. V. and Hari, K. V. S., "Performance analysis of subspace based methods," in IEEE Ath ASSP Workshop on Spectral Estimation 8 Modeling, (Minnesota, MN), pp. 92-97, August 1988.

Bhaskar Rao, D. V. and Hari, K. V. S., "Quality of low rank approximants obtained using SVD and some applications," in Proc. 21" Annual Asilomar Conf. on Signals, Systems and Computers, (Pacific Grove, CA), pp. 784-787, November 1987.

Bhaskar Rao, D. V. and Hari, K. V. S., "Statistical performance analysis of Minimum-Norm method," IEE Proc., vol. 136, Pt.F, pp. 125-134, June 1989.

Bhaskar Rao, D. V. and Hari, K. V. S., "Statistical performance analysis of Minimum-Norm method," in Proc. IEEE ICASSP'89, (Glasgow, UK), pp. 2760-2763, May 1989.

Bienvenu, G. and Kopp, L., "Principle de la Goniometric passive adaptive," in Proc. 7'eme Colloque GRESIT, (Nic, France), pp. 106/1-106/10, 1979.

Burg, J. P., "Maximum entropy spectral analysis," in Proc. 97th Meeting of the Society of Exploration Geophysicists, pp. 34-41, 1967.

Capon, J., "High-resolution frequency-wavenumber spectrum analysis," Proc. IEEE, vol. 57, pp. 1408-1418, 1969.

Clergeot, H., Tressens, S., and Ouamri, A., "Performance of high resolution frequencies estimation methods compared to the Cramer-Rao bounds," IEEE 
Trans. Acoustics, Speceh, and Signal Processing, vol. ASSP-37, pp. 1703-1720, November 1989.

Cooley, J. W. and Turkey, J. W., "An algorithm for the machine calculation of fourier series," Math. Comput, vol. 19, pp. 297-301, 1965.

Farrier, D. R., Jeffries, D. J., and Mardani, R., "Perturbation analysis of the MUSIC algorithm," in Proc. IEEE ICASSP'88, (New York, NY), pp. 28732876, April 1988.

Farrier, D. R., Jeffries, D. J., and Mardani, R., "Theoretical performance prediction of the MUSIC algorithm," IEE Proc., Pt. F, vol. 135, pp. 216-225, June 1988.

Foka, R., "Comparison of high resolution spectral methods based on SVD," in Proc. IEEE ICASSP'87, (Dallas, TX), pp. 2233-2236, April 1987.

Foka, R. and Kung, S. Y., "A novel optimum energy solution in iterative constrained restoration," in Proc. EUSIPCO'88, pp. 1041-1044, Science Publishers B. V., 1986.

Friedlander, B., "A sensitivity analysis of the MUSIC algorithm," in Proc. IEEE ICASSP'89, (Glasgow, UK), pp. 2811-2814, May 1989.

Hua, Y. and Sarkar, T. K., "Matrix pencil method and its performance," in Proc. IEEE ICASSP'88, (New York, NY), pp. 2476-2479, April 1988.

Hua, Y. and Sarkar, T. K., "Perturbation analysis of TK method for harmonic retrieval problems," IEEE Trans. Acoustics, Speech, and Signal Processing, vol. ASSP-36, pp. 228-240, February 1988.

Jackson, L. B., Digital Filters and Signal Processing. Boston, MA: Kluwer Academic Publishers, 1986.

Johnson, D., "The application of spectral estimation methods to bearing estimation problem," Proc. IEEE, vol. 70, pp. 1018-1028, 1982. 
Kaveh, M. and Barabell, A. J., "The statistical performance of the MUSIC and the Minimum-Norm algorithms in resolving plane-waves in noise," IEEE Trans. Acoustics, Speech, and Signal Processing, vol. ASSP-34, pp. 331-340, April 1986.

Kot, A. C., Perturbation Analysis of SVD-Based Methods. PhD thesis, University of Rhode Island, Kingston, RI, 1989.

Kot, A. C., Parthasarathy, S., Tufts, D. W., and Vaccaro, R. J., "Statistical performance of single sinusoid frequency estimation in white noise using statevariable balancing and Linear Prediction," IEEE Trans. Acoustics, Speech, and Signal Processing, vol. ASSP-35, pp. 1639-1642, November 1987.

Kumaresan, R. and Tufts, D. W., "Estimating the angles of arrival of multiple plane waves," IEEE Trans. Aerosp., Electron. Syst., vol. AES-19, pp. 134-139, January 1983.

Kung, S. Y., Arun, K. S., and Bhaskar Rao, D. V., "State-space and singularvalue decomposition-based approximation methods for the harmonic retrieval problem," J. Opt. Soc. Am., vol. 73, pp. 1799-1811, December 1983.

Kung, S. Y., Bhaskar Rao, D. V., and Arun, K. S., "New state space and singular value decomposition based approximate modeling methods for harmonic retrieval," in IEEE 2nd ASSP Workshop on Spectral Estimation \& Modeling, pp. 266-271, August 1983.

Lancaster, P. and Tismentsky, M., The Theory of Matrices. New York, NY: Academic Press, second ed., 1978.

Le Cadre, J. and Ravazzola, P., "Approximated stochastic realization and model reduction methods applied to array processing by means of state space models," in Proc. IEEE ICASSP'89, (Glasgow, UK), pp. 2601-2604, May 1989. 
Li, F. and Vaccaro, R. J., "Analysis of music and min-norm for arbitrary array geometry," IEEE Trans. Aerosp., Electron. Syst., vol. AES-27, January 1991.

Li, F. and Vaccaro, R. J., "MUSIC performance prediction by matrix approximation at high SNR," in Proc. 1989 Conf. Information Sciences and Systems, (The Johns Hopkins University, Baltimore, MD), pp. 447-481, March 1989.

Li, F. and Vaccaro, R. J., "A unified performance analysis of subspace-based polynomial-rooting algorithms for doa estimation," in Proc. 1990 Conf. Information Sciences and Systems, (Princeton, NJ), March 1990.

Li, F., Vaccaro, R. J., and Tufts, D. W., "Min-Norm Linear Prediction for arbitrary sensor array," in Proc. IEEE ICASSP'89, (Glasgow, UK), pp. 26132616, May 1989.

Li, F., Vaccaro, R. J., and Tufts, D. W., "Unified performance analysis of subspace-based estimation algorithms," in Proc. IEEE ICASSP'90, (Albuquerque, NM), pp. 2575-2578, April 1990.

Mayrargue, S., "ESPRIT and TAM (toeplitz approximation method) are theoretically equivalent," in IEEE Proc. ICASSP'88, (New York, NY), pp. 24562459, April 1988.

Mayrargue, S. and Jouveau, J. P., "On equivalence between esprit and tam (toeplitz approximation method), lead to an improved tam," in Proc. EUSIPCO'88, pp. 55-58, Science Publishers B. V., 1988.

Ouibrahim, H., Weiner, D. D., and Sarkar, T. K., "A general approach to direction-finding," in Proc. IEEE MILCON, pp. 41.4.1-41.4.5, 1986.

Ouibrahim, H., Weiner, D. D., and Sarkar, T. K., "A general approach to direction-finding," IEEE Trans. Acoustics, Speech, and Signal Processing, vol. ASSP-36, pp. 610-612, April 1988. 
Ouibrahim, H., Weiner, D. D., and Sarkar, T. K., "Matrix pencil approach to angle of arrival estimation, ${ }^{n}$ in Proc. $20^{\text {th }}$ Asilomar Conf. on Signals, Systems and Computers, (Pacific Grove, CA), pp. 203-206, November 1986.

Paulaj, A., Roy, R., and Kailath, T., "Estimation of signal parameters via rotational invariance techniques - ESPRIT, " in Proc. $19^{\text {th }}$ Asilomar Conf. on Signals, Systems and Computers, (Pacific Grove, CA), pp. 83-89, November 1985.

Paulaj, A., Roy, R., and Kailath, T., "A subspace rotation approach to parameter estimation,” Proc. IEEE, vol. 70, pp. 1044-1045, July 1986.

Pisarenko, V. F., "The retrieval of harmonics from a covariance function," Geophys. J. Royal Astronom. Soc., vol. 33, pp. 347-366, 1976.

Porat, B. and Friedlander, B., "An accuracy analysis of the Kumaresan-Tufts method for estimating complex damped exponentials," IEEE Trans. Acoustics, Speech, and Signal Processing, vol. ASSP-35, pp. 231-235, February 1987.

Porat, B. and Friedlander, B., "Analysis of the asymptotic relative efficiency of the MUSIC algorithm," IEEE Trans. Acoustics, Speech, and Signal Processing, vol. ASSP-36, pp. 532-543, April 1988.

Porat, B. and Friedlander, B., "On the asymptotic relative efficiency of the MUSIC algorithm," in Proc. IEEE ICASSP'88, (New York, NY), pp. 23762379, April 1988.

Prony, R., "Essai experimental et analytique etc.," L'ecole Polytechnique, pp. 24-76, 1795.

Roy, R., ESPRIT - Estimation of Signal Parameters Via Rotational Invariance Techniques. PhD thesis, Stanford University, Stanford, CA, 1987.

Roy, R. and Kailath, T., "ESPRIT - estimation of signal parameters via rotational invariance techniques," IEEE Trans. Acoustics, Speech, and Signal Processing, vol. ASSP-37, pp. 984-995, July 1989. 
Roy, R. and Kailath, T., "Total least-squares ESPRIT," in Proc. $21^{\text {st }}$ Asilomar Conf. on Signals, Systems and Computers, (Pacific Grove, CA), pp. 580-584, November 1987.

Roy, R., Paulaj, A., and Kailath, T., "Comparative performance analysis of ESPRIT and MUSIC direction-of-arrival estimation," in Proc. $20^{\text {th }}$ Asilomar Conf. on Signals, Systems and Computers, (Pacific Grove, CA), pp. 580-584, November 1986.

Roy, R., Paulaj, A., and Kailath, T., "Comparative performance analysis of ESPRIT and MUSIC direction-of-arrival estimation," in Proc. IEEE ICASSP'87, (Dallas, TX), pp. 2344-2347, April 1987.

Roy, R., Paulaj, A., and Kailath, T., "Direction-of-arrival estimation by subspace rotation methods - ESPRIT," in Proc. IEEE ICASSP'86, (Tokyo, Japan), pp. 47.2.1-47.2.4, April 1986.

Roy, R., Paulaj, A., and Kailath, T., "Estimation of signal parameters via rotational invariance techniques - ESPRIT," in Proc. IEEE MILCON, pp. 41.6.1-41.6.5, 1986.

Schmidt, R. O., "Multiple emitter location and signal parameter estimation," in Proc. RADC Spectral Estimation Workshop, (Griffiss AFB, NY), pp. 243258, 1979.

Schmidt, R. O., A Signal Subspace Approach to Multiple Emitter Location and Spectral Estimation. PhD thesis, Stanford University, Stanford, CA, 1981.

Shan, T. J., Wax, M., and Kailath, T., "On spatial smoothing for direction-ofarrival estimation for coherent signals," IEEE Trans. Acoustics, Speech, and Signal Processing, vol. ASSP-33, pp. 806-811, August 1985.

Sharman, K. and Durrani, T. S., "A comparative study of modern eigenstructure methods for bearing estimation - a new high performance approach," in Proc. $2^{\text {th }}$ IEEE Conf., on Decision and Control, pp. 1737-1742, 1986. 
Spielman, D., Paulraj, A., and Kailath, T., "Performance analysis of the MUSIC algorithm," in Proc. IEEE ICASSP'86, (Tokyo, Japan), pp. 1909-1912, April 1986.

Stewart, G. W., "Perturbation theory for the singular value decomposition," in Proc. 2nd International Workshop on SVD and Signal Processing, (Kingston, RI), June 1990.

Stewart, G. W., "Stochastic perturbation theory," in UMIACS-TR-SS-76, (College Park, MD), pp. 1-24, October 1988.

Stoica, P. and Nehorai, A., "MUSIC, Maximum Likelihood and Cramer-Rao bound," in Proc. IEEE ICASSP'88, (New York, NY), pp. 2296-2299, April 1988.

Stoica, P. and Nehorai, A., "MUSIC, Maximum Likelihood and Cramer-Rao bound," IEEE Trans. Acoustics, Speech, and Signal Processing, vol. ASSP-37, pp. 720-741, May 1989.

Stoica, P. and Nehorai, A., "MUSIC, Maximum Likelihood and Cramer-Rao bound: Further results and comparisons," in Proc. IEEE ICASSP'89, (Glasgow, UK), pp. 2605-2608, April 1989.

Tufts, D. W. and Kumaresan, R., "Estimation of frequencies of multiple sinusoids: Making linear prediction perform like maximum likelihood," Proc. IEEE, vol. 70, pp. 975-989, September 1982.

Tufts, D. W., Vaccaro, R. J., and Kot, A. C., "Analysis of estimation of signal parameters by linear prediction at high SNR using matrix approximation," in Proc. IEEE ICASSP'89, (Glasgow, UK), May 1989.

Vaccaro, R. J., Tufts, D. W., and Boudreaux-Bartels, G. F., SVD and Signal Processing, ch. Advances in Principal Component Signal Processing, pp. 115148. E.F. Deprettere, ed., North-Holland, 1988. 
Zoltowski, M. D., "Novel techniques for estimation of array signal parameters based on matrix pencil, subspace rotations, and total least squares," in Proc. IEEE ICASSP'88, (New York, NY), pp. 2861-2864, April 1988. 